\title{
THE RANK 3 PERMUTATION REPRESENTATIONS OF THE FINITE CLASSICAL GROUPS
}

BY

\author{
WILLIAM M. KANTOR AND ROBERT A. LIEBLER
}

\begin{abstract}
The permutation representations in the title are all determined, and no surprises are found to occur.
\end{abstract}

1. Introduction. A group $G$ has rank 3 in its permutation representation on the cosets of a subgroup $K$ if there are exactly $3(K, K)$-double-cosets; that is, if $K$ has exactly 3 orbits on the set $G / K$ of $K$-cosets. Such permutation representations have been studied a great deal during the past 15 years; classical groups have been intensively studied for more than a century. The purpose of this paper is to relate these two areas, by proving the following results.

THEOREM 1.1. Let $M$ be one of the groups

$$
S p(2 m-2, q), \quad \Omega^{ \pm}(2 m, q), \quad \Omega(2 m-1, q) \text { or } S U(m, q)
$$

for $m \geqslant 3$ and $q$ a prime power. Let $M \unlhd G$ with $G / Z(M) \leqslant \operatorname{Aut}(M / Z(M))$. Assume that $G$ acts as a primitive rank 3 permutation group on the set $X$ of cosets of $a$ subgroup $K$ of $G$. Then (at least) one of the following holds up to conjugacy under $\operatorname{Aut}(M / Z(M))$.

(i) $X$ is an $M$-orbit of singular (or isotropic) points.

(ii) $X$ is an $M$-orbit of maximal totally singular (or isotropic) subspaces and $M=S p(4, q), S U(4, q), S U(5, q), \Omega^{-}(6, q), \Omega^{+}(8, q)$ or $\Omega^{+}(10, q)$.

(iii) $X$ is any $M$-orbit of nonsingular points and $M=S U(m, 2), \Omega^{ \pm}(2 m, 2)$, $\Omega^{ \pm}(2 m, 3)$ or $\Omega(2 m-1,3)$.

(iv) $X$ is either orbit of nonsingular hyperplanes of $M=\Omega(2 m-1,4)$ or $\Omega(2 m-1,8)$ (where $G=\Omega(2 m-1,8) \cdot 3$ in the latter case).

(v) $M=S U(3,3), K \cap M=P S L(3,2)$ (Mitchell [40], Suzuki [56]).

(vi) $M=S U(3,5), K \cap M=3 \cdot A_{7}$ (Mitchell [40], Higman [24]).

(vii) $M=S U(4,3), K \cap M=4 \cdot P S L(3,4)$ (Hartley [21], McLaughlin [38]).

(viii) $M=S p(6,2), K=G_{2}(2)$ (Edge [15], Frame [19]).

(ix) $M=\Omega(7,3), K \cap M=G_{2}(3)$.

(x) $M=S U(6,2), K \cap M=3 \cdot P S U(4,3) \cdot 2$ (Fischer [17]).

Received by the editors December 19, 1977 and, in revised form, June 13, 1980. Presented at the AMS Regional Conference on Representations of Finite Chevalley Groups in Madison, Wisconsin, August 1977.

1980 Mathematics Subject Classification. Primary 20G40, 20C30; Secondary 51E20, 05C25.

${ }^{1}$ The research of both authors was supported in part by NSF Grants. 
Theorem 1.2. Let $M=P S L(n, q) \leqslant G \leqslant$ Aut $M$. Assume that $G$ acts as a primitive rank 3 permutation group on the set $X$ of cosets of a subgroup $K$ of $G$. Then (at least) one of the following occurs up to conjugacy under Aut $M$.

(i) $X$ is the set of lines for $M, n \geqslant 4$.

(ii) $M=P S L(2,4) \cong P S L(2,5),|X|=\left(\begin{array}{l}5 \\ 2\end{array}\right)$,

$$
\begin{aligned}
& M=P S L(2,9) \cong A_{6},|X|=\left(\begin{array}{l}
6 \\
2
\end{array}\right), \\
& M=P S L(4,2) \cong A_{8},|X|=\left(\begin{array}{l}
8 \\
2
\end{array}\right), \text { or } \\
& G=P \Gamma L(2,8),|X|=\left(\begin{array}{l}
9 \\
2
\end{array}\right) . \\
& \text { (iii) } M=P S L(3,4), M \cap K=A_{6} . \\
& \text { (iv) } M=P S L(4,3), M \cap K=P S p(4,3) .
\end{aligned}
$$

The reader is cautioned that isomorphisms between, and automorphisms of, the various groups allow many different ways of viewing some of these cases. Specifically, the isomorphisms $P S p(4, q) \cong \Omega(5, q), \quad P S U(4, q) \cong P \Omega^{-}(6, q)$, $P S L(4, q) \cong P \Omega^{+}(6, q)$ and $P S U(4,2) \cong P S p(4,3)$ lead to numerous representations under (1.1i). Similarly, a triality automorphism of $P \Omega^{+}(8, q)$ can be applied to (1.1iii) and a polarity automorphism can be applied to (1.2i).

The 2-transitive representations for $G$ have been determined by Curtis, Kantor and Seitz [9]; from this result, the imprimitive rank 3 representations of $G$ can be obtained (see $§ 11$ ). Our approach is similar to theirs, especially their note "Added in proof". Both arguments rely heavily on the degrees of the nonprincipal irreducible constituents of $1_{B}^{G}$, where $B$ is a Borel subgroup (and $G$ is semilinear on the natural module $V$ for $M$ ). All such degrees are divisible by $p$, with only 3 exceptions for $G=S p(2 n, 2)$. This fact, which follows from Hoefsmit [26], was not available in [9]; on the other hand, 2-transitivity permitted counting arguments in [9] which have no parallels here. (Only if $G=S p(6,2)$ or $S p(8,2)$ are we able to use the standard rationality conditions for the parameters of a rank 3 group.) When combined with a result of Seitz [50], this divisibility implies that $1_{K}^{G}$ and $1_{B}^{G}$ have exactly one nonprincipal constituent $\chi$ in common. In almost all cases, this fact by itself is strong enough to determine $K$. (A more general classification based upon this idea is found in (11.1).) Computations within the Weyl group yield strong transitivity properties for the action of $K$ on $V$. Now a method of Perin [46] applies and $K$ can be determined. The analogues of (1.1) and (1.2) for the remaining Chevalley groups seem blocked by the problem of finding all subgroups transitive (or almost transitive) on one or more classes of parabolic subgroups.

The only other results of this type are the beautiful theorem of Seitz [51] which proves the same result for $q$ large relative to $m$, and the determination by Bannai [2] (resp., Cohen [5]) of all representations of rank at most 5 of $A_{n}$ and $S_{n}$ (resp., of rank 3 of all complex reflection groups). It seems quite difficult to extend our arguments to higher rank representations.

The paper is organized as follows. In $\$ 2$ we present some notation and numerous preliminary results. Characters of the Weyl groups of type $B_{n}$ and $D_{n}$ are discussed in $\S 3$, and applied to $G$ in $\S 4$; this is where some of Hoefsmit's results are described.

Highly transitive subgroups of $G$ are crucial to our approach. These are studied for small dimensions in $\S 5$, and for large dimensions in $§ 6$ (using Perin’s method). 
We obtain a number of extensions of Seitz's flag-transitive theorem. For example, subgroups transitive on points, lines and planes (if planes exist) are all determined; mere transitivity on lines suffices for unitary geometries of dimension at least 8 . On the other hand, $\S \S 5$ and 6 contain many technical results with hypotheses tailored to the proof of (1.1). The proofs tend to be involved and unpleasant, due in part to several interesting (and annoying) exceptions. These exceptions, which arise in $\S 5$, are carried over to $\S 6$, where reductions are made to small dimensional situations.

The proof of (1.1) occupies $\$ \S 7-9$. The basic ideas in the proof of (1.1) can be clearly seen in $\$ \S 7-8$. In particular, the precision with which the Pigeon-Hole Principle applies is evident in (8.1). In $\$ 9$ we deal with two exceptional and tedious cases: $S p(2 n, 2)$, where $1_{B}^{G}$ contains two nonprincipal characters of odd degree; and the possibility of graph automorphisms arising in the case of $P \Omega^{+}(8, q)$.

The proof of (1.2) appears in $\$ 10$. This result is much simpler than (1.1), and its proof is relatively self-contained. The reader may wish to start the paper with that section.

One of the features of our proofs of (1.1) and (1.2) is that the rank 3 hypothesis is not fully used. Consequently, other subgroups of classical groups are able to occur which, from our point of view, behave very much like stabilizers in rank 3 representations. This is discussed in $\$ 11$, where more general technical results are indicated (a generalization of much of (1.2) having already been proved in (10.1)). In $\$ 11$ we have also listed the known examples of rank 3 representations of other Chevalley groups.

Finally, $\$ 12$ represents a change of topic, though not of method. In [25], Higman and McLaughlin studied subgroups $K$ of $\Gamma S p(V)$ or $\Gamma U(V)$ which are rank 3 on the set of points of $V$. A complete determination was made by Perin [46], except in the case $S p(2 n, 2)$. (For a stronger unitary result, see our (6.1).) The orthogonal case was examined by Stark [54] for odd characteristic and small dimensions. By imitating Perin [46] and some portions of $\S 6$, we will prove the following result.

THEOREM 1.3. Suppose that $K \leqslant \Gamma O^{ \pm}(d, q), d \geqslant 5$, and that $K$ has rank 3 on points. If $d$ is odd, assume that $q$ is odd. Then $K \geqslant \Omega^{ \pm}(d, q)$.

We are indebted to Dr. Jan Saxl for his invaluable assistance with parts of §3. We are also grateful to Professor Harriet Pollatsek for a number of helpful remarks.

Finally, we are very grateful to the referee for his diligence and his many constructive suggestions and comments.

\section{Preliminaries.}

A. Notation. Let $V$ be a finite vector space of characteristic $p$ equipped with a symplectic, unitary, or orthogonal geometry. The corresponding Chevalley (or twisted) group $\operatorname{Chev}(V)$ is $S p(V), S U(V)$, or $\Omega^{ \pm}(V)$ (or just $\Omega(V)$ if $\operatorname{dim} V$ is odd). Note that $\operatorname{Chev}(V)$ may have a nontrivial center.

The group of semisimilarities of $V$ will be denoted by $\Gamma(V)=\Gamma S p(V), \Gamma U(V)$ or $\Gamma O^{ \pm}(V)$. This is the group of semilinear transformations $g$ of $V$ such that $f\left(u^{g}, v^{g}\right)=c f(u, v)^{\sigma}\left(\right.$ or $\left.f\left(u^{g}\right)=c f(u)^{\sigma}\right)$ for some scalar $c$, some field automorphism $\sigma$, and all $u, v \in V$; here, $f$ is the sesquilinear (or quadratic) form on $V$. (See 
Dieudonné [13, I.10, I.16].) Modulo scalars, $\Gamma(V)$ is the automorphism group of $\operatorname{Chev}(V)$ in almost all cases. The only exceptions occur for $\operatorname{Sp}\left(4,2^{e}\right)$ and $\Omega^{+}(8, q)$ (Carter [3, Chapter 12]).

$\operatorname{Chev}(V)$ is generated by "long root elements": nontrivial transvections in the symplectic and unitary cases; in the orthogonal cases (with $\operatorname{dim} V>4$ ), elements of order $p$ having $L^{\perp}$ as an eigenspace, for some totally singular line $L$. For more information, see [13], [3] or [33].

There is a natural isomorphism $\operatorname{Sp}(2 n, q) \cong \Omega(2 n+1, q)$ for $q$ even. We will therefore only have to consider whichever one of these groups is most convenient in a given situation, usually without even mentioning the other group. We will frequently make use of the isomorphisms $\Omega(5, q) \cong P S p(4, q)$, $P \Omega^{+}(6, q) \cong P S L(4, q)$ and $P \Omega^{-}(6, q) \cong P S U(4, q)$.

Totally singular (or isotropic) $i$-spaces will simply be called " $i$-spaces". All other subspaces will be modified by suitable adjectives. Points, lines and planes are, of course, just 1-spaces, 2-spaces and 3-spaces. The letters $x, y, z$ will be reserved for points, $L$ for lines, and $E$ for planes. By abuse of language, we will write $x \in S$ in place of $x \subset S$.

The maximum dimension of an $i$-space will be denoted by $n$.

An $(i, j)$-flag consists of an $i$-space and a $j$-space containing it; also, in the $\Omega^{+}(2 n, q)$ case, there are $(n, n)$-flags, consisting of two $n$-spaces meeting in an $n-1$-space. A flag for $S p(V), S U(V)$ and $O^{ \pm}(V)$ is a nested sequence of $i$-spaces, one for each $i=1, \ldots, n$; a flag for $\Omega^{+}(2 n, q)$ is a sequence $\left(V_{1}, \ldots, V_{n-2}, V_{n}, V_{n}^{\prime}\right)$ consisting of nested $i$-spaces for $i=1, \ldots, n-2$, along with two $n$-spaces $V_{n}, V_{n}^{\prime} \supset V_{n-2}$ meeting in an $n-1$-space.

Throughout $\S \S 2-9, G$ will denote a group lying between $\operatorname{Chev}(V)$ and $\Gamma(V)$, while $K$ will be a subgroup of $G$.

If $W \subseteq V$, then $K_{W}$ and $C_{K}(W)$ are the set-wise and vector-wise stabilizers of $W$ in $K$; the semilinear group induced by $K_{W}$ on $W$ is denoted $K_{W}^{W} \cong K_{W} / C_{K}(W)$. Also, $W^{K}$ is the orbit of $W$ under $K ;[V, K]=[K, V]=\left\{v^{k}-v \mid k \in K\right\} ; K^{\prime}$ is the commutator subgroup of $K, K^{(\infty)}$ is the last term of the commutator series for $K$, $Z(K)$ is the center of $K$, and $O_{p}(K)$ is the largest normal $p$-subgroup of $K$. If $H$ is a group, $n \cdot H$ is an extension of $H$ by a group of order $n$, while $H \cdot n$ is $H$ with an automorphism of order $n$ adjoined.

B. Some subgroups of classical groups. We will require a number of properties of classical groups related to generation and transitivity. We will also make very frequent use of the structure of the most obvious parabolic subgroups $[9, \S 3]$ :

LeMma 2.1. Let $x$ be a point, and set $Q=O_{p}\left(G_{x}\right)$, so that $Q$ centralizes $x^{\perp} / x$. Let $Q_{1}$ be the group of transvections in $Q$.

(i) The representations of $C_{G}(x)\left(\right.$ resp., $\left.G_{x}\right)$ on $Q / Q_{1}$ and $x^{\perp} / x$ are isomorphic (resp., projectively isomorphic), via $g Q_{1} \rightarrow\left[V, g Q_{1}\right] / x$.

(ii) In the orthogonal case, singular vectors in $x^{\perp} / x$ correspond to long root elements in $Q$; nonsingular vectors correspond to nontrivial elements $g \in Q$ having $\operatorname{rad}[V, g] \neq[V, g]$. 
(iii) If $V$ is unitary, or symplectic of odd characteristic, then $Q^{\prime}=Q_{1}=\Phi(Q)$, and the commutator function on $Q \times Q$ induces a nondegenerate alternating bilinear form on $Q / Q_{1}$.

Lemma 2.2. Suppose that $K$ is a subgroup of $\Gamma(V)$ containing a Sylow p-subgroup of $\operatorname{Chev}(V)$. Then either $K \geqslant \operatorname{Chev}(V)$, or $K$ stabilizes some totally singular subspace.

Proof. This is due to Tits (see Seitz [51, (4.6)]).

The preceding lemma is, in effect, concerned with subgroups of Chevalley groups generated by root groups, even in the infinite case. We will require much more detailed information concerning groups generated by long root groups.

LEMMA 2.3. Let $K$ be a subgroup of $\Gamma(V)$ which is primitive (as a linear group) on $V / \operatorname{rad} V$, where $V$ does not have type $\Omega^{+}(4, q)$. Assume that the subgroup $K^{*}$ generated by all long root elements of $K$ is nontrivial. Then either (i) $K^{*}=\left\langle t^{K^{*}}\right\rangle$ for some long root element $t$, or (ii) $V$ has type $\Omega(5, q)$ with $q$ even, and there is a nonsingular $K$-invariant hyperplane on which $K^{*}$ induces $\Omega^{+}(4, q)$.

Proof. Clearly $O_{p}\left(K^{*}\right)=1$. By Cooperstein [7, (3.8)], there are subgroups $K_{1}, \ldots, K_{r}$ of $K^{*}$ such that $K^{*}=K_{1} \cdots K_{r},\left[K_{i}, K_{j}\right]=1$ for $i \neq j, K_{i}=\left\langle t_{i}^{K_{i}}\right\rangle$ for some long root element $t_{i}$, and $\cup K_{i}$ contains all long root elements of $K$. We must show that $r=1$ unless (ii) holds.

Suppose that $r>1$, and consider $A_{i}=\left[V, t_{i}\right]$.

If $V$ is symplectic or unitary, then $A_{1}$ and $A_{2}$ are points which are perpendicular since $\left[t_{1}, t_{2}\right]=1$. Thus, the subspaces $\left[V, K_{i}\right]$ are pairwise orthogonal. Since $V$ is spanned by these subspaces, while $K$ permutes these subspaces, $K$ cannot be primitive.

If $V$ is orthogonal, then $A_{1}$ and $A_{2}$ are lines. This time, $\left[t_{1}, t_{2}\right]=1$ implies that either $A_{2} \subseteq A_{1}^{\perp}$ or $A_{1} \cap A_{2} \neq 0$. Consider the latter possibility. Since $O_{p}\left(K_{i}\right)=1$, $W=\left\langle A_{1}, A_{1}^{g}\right\rangle$ is a nonsingular 4-space for some $g \in K_{1}$. Since $A_{1}^{\perp} \cap A_{1}^{g}=0$, it follows that, if $h \in G$, then $W^{h}=W$ or $W^{h} \subset W^{\perp}$. Then $V=\langle W, \operatorname{rad} V\rangle$ by the primitivity of $K$.

Consequently, if we exclude case (ii), then the subspaces $\left[K_{i}, V\right]$ are again pairwise orthogonal and we obtain the same contradiction as before.

THEOREM 2.4. Suppose that $n \geqslant 2, V$ is not of type $\Omega^{+}(4, q)$, and $K$ is a subgroup of $\Gamma(V)$ which is point-transitive and contains a long root element. Then one of the following holds:

(i) $K \geqslant \operatorname{Chev}(V)$;

(ii) $K \unrhd G_{2}(q)$ and $\Gamma(V)=\Gamma O(7, q)$;

(iii) $K \unrhd N$ with $-1 \in Z(N)$ and $N /\langle-1\rangle$ conjugate in Aut $P \Omega^{+}(8, q)$ to one of the usual subgroups $P \Omega(7, q)$;

(iv) $K \unrhd S U(m, q)$ and $\Gamma(V)=\Gamma O^{\varepsilon}(2 m, q)$ (with $\varepsilon=+$ iff $m$ is even $)$; or

(v) $O_{3}(K) \$ Z(K)$ and $K$ has an extraspecial subgroup of order $3^{3}$, where $K<\Gamma O^{-}(6,2)$. 
Proof. This follows from (2.3) and a check of the lists in [33].

REMARKS. That the groups in (iii) are point-transitive follows from the fact that the usual subgroup $\Omega(7, q)$ is transitive on each class of 4-spaces of $V$. Aut $P \Omega^{+}(8, q)$ will enter into our arguments in a number of other situations.

There will also be other occasions when [33] will be quoted. Unfortunately, the lists in that paper are too long to reproduce here. Another use of [33] is the following lemma (compare Stark [53]).

LeMMA 2.5. Let $K$ be a primitive subgroup of $\Gamma O^{ \pm}(m, q)$, where $q$ is odd and $m \geqslant 5$. Let $x$ be a point, and $b_{1}, b_{2} \in x^{\perp}$ with $\operatorname{dim}\left\langle x, b_{1}, b_{2}\right\rangle=3$. Assume that $\left|C_{K}\left(\left\langle x, b_{i}\right\rangle^{\perp}\right)\right|=q$ for $i=1,2$. Then either $K \geqslant \Omega^{ \pm}(m, q)$ or $K \unrhd 2 \cdot \Omega(7, q)$, inside $\mathrm{\Gamma O}^{+}(8, q)$.

Proof. Write $A(x)=C_{K}(x) \cap C_{K}\left(x^{\perp} / x\right), V(x)=[V, A(x)]$ and $\Omega=x^{K}$. Note that $V=\langle\Omega\rangle$.

If $K$ contains long root elements, then (2.3) and [33] provide a short list of possibilities. Since $\left|C_{K}\left(\left\langle x, b_{1}\right\rangle^{\perp}\right)\right|=q$, the only ones that occur are those stated in the lemma.

From now on, we will assume that $K$ contains no long root elements. By (2.1), $V(x) / x$ is an anisotropic 2-space and $|A(x)|=q^{2}$.

Let $y \in \Omega-x^{\perp}$. If $0 \neq b \in V(x) \cap y^{\perp}$, there is a nonzero vector $d \in V(y) \cap x^{\perp}$ such that exactly one of $(b, b),(d, d)$ is a square in $G F(q)$. Then $\langle b, d\rangle$ is nonsingular, and hence so is $W=\langle x, y\rangle \oplus\langle b, d\rangle$. Write

$$
K_{1}=\left\langle C_{K}\left(\langle x, b\rangle^{\perp}\right), C_{K}\left(\langle y, d\rangle^{\perp}\right)\right\rangle \text {. }
$$

Then $K_{1}^{W}$ is generated by nonconjugate groups of order $q$, and hence is $\Omega(W)$. Also, $K_{1}$ centralizes $W^{\perp}$. Since $K$ has no long root elements, $W$ must have type $\Omega^{-}(4, q)$. Moreover, $V\left(x^{\prime}\right) \subset W$ and $\operatorname{dim} V(x) \cap V\left(x^{\prime}\right) \geqslant 2$ for each point $x^{\prime}$ of $W$ (since a Sylow $p$-subgroup of $K_{1}$ has order $q^{2}$ ).

By primitivity, there is a point $z \in \Omega-\left(W \cup W^{\perp}\right)$. We may assume that $z \notin x^{\perp}$. As above, $\operatorname{dim} V(x) \cap V(z)=2$. Thus, $Z=\langle W, V(z)\rangle$ has dimension 5. Clearly, $H=\langle A(x), A(y), A(z)\rangle$ acts on $Z$, while centralizing $Z^{\perp}$.

By Mitchell [41], $Z$ cannot be nonsingular. (This also follows readily from the fact that $K_{1}$ would be transitive on the lines of $Z$.) Set $r=\operatorname{rad} Z$ and $T=O_{p}\left(C_{H}(Z / r)\right)$. Both $K_{1}$ and $\langle A(x), A(z)\rangle$ induce $\Omega^{-}(4, q)$ on $Z / r$, and they fix different hyperplanes of $Z$. Thus, $T \neq 1$. Here, $T$ consists of transvections of $Z$ with direction $r$. Since $K_{1}$ acts on $T$, we must have $|T|=q^{4}$. But now some element of $T$ fixes $x$ while moving $V(x)$. This contradiction proves the lemma.

THEOREM 2.6. Suppose that $n \geqslant 2$ and that $V$ is not of type $\Omega^{+}(4, q)$. Let $K$ be $a$ flag-transitive subgroup of $\Gamma(V)$ which does not contain $\operatorname{Chev}(V)$. Then one of the following holds:

(i) $\mathrm{K}$ is $\mathrm{A}_{7}$ or $\mathrm{S}_{7}$, inside $\mathrm{O}^{+}(6,2)$;

(ii) $K$ is $A_{6}$, inside $\operatorname{Sp}(4,2)$;

(iii) $K$ is a semidirect product of an extraspecial group of order 32 with $A_{5}, S_{5}$ or a Frobenius group of order 20, inside $\Gamma \operatorname{Sp}(4,3)$; 
(iv) $K$ has a normal subgroup which is a semidirect product of an elementary abelian group of order 16 with $A_{5}, S_{5}$ or a Frobenius group of order 20, inside $\Gamma O(5,3)$;

(v) $K \unrhd 2 \cdot P S L(3,4) \cdot 2$, inside $\mathrm{\Gamma O}^{-}(6,3)$; or

(vi) $K \unrhd 4 \cdot P S L(3,4) \cdot 2$, inside $\Gamma U(4,3)$.

Proof. Seitz [50]. (Note that (iii) and (iv) are related via the isomorphism $P S p(4,3) \cong \Omega(5,3)$, while (v) and (vi) are related via the isomorphism $P \Omega^{-}(6,3) \cong P S U(4,3)$.)

LEMMA 2.7. Let $K \leqslant P \Gamma L(3, q)$.

(i) If $K$ is point-transitive on $P G(2, q)$, then $K \geqslant P S L(3, q)$ or $K$ has a normal irreducible cyclic subgroup of order dividing $q^{2}+q+1$.

(ii) If $K$ has 2 point-orbits and 3 flag-orbits, then $K$ fixes a point or line, or else $K$ is $A_{6}$ or $S_{6}$ stabilizing a hyperoval of $P G(2,4)$.

(iii) If $K$ has 3 point-orbits and at most 6 flag orbits, then $K$ has 6 flag-orbits and fixes a point or a line, or an oval or its dual.

(iv) If $L, L^{\prime}$ are lines and both $K_{L}^{L}$ and $K_{L^{\prime}}^{L^{\prime}}$ are transitive, then $L^{\prime} \in L^{K}$.

Proof. Mitchell [40] and Hartley [22].

LEMMA 2.8. Let $K \leqslant P \Gamma U(3, q)$.

(a) If $K$ is transitive on isotropic points, then one of the following holds:

(i) $K \geqslant P S U(3, q)$;

(ii) $K \leqslant P \Gamma U(3,2)$;

(iii) $K^{\prime}$ is $P S L(3,2)$ inside $P \Gamma U(3,3)$; or

(iv) $K^{\prime}$ is $A_{7}$ inside $P \Gamma U(3,5)$.

(b) If $K$ has 2 orbits on isotropic points, then one of the following holds:

(i) $K$ fixes a line;

(ii) $K \leqslant P \Gamma O(3, q)$;

(iii) $K \cap P S U(3, q)$ is inside one of the groups appearing in (a iii) or (a iv); or

(iv) $K$ lies in the normalizer of a Sylow 7-subgroup of $P \Gamma U(3,5)$.

Proof. Mitchell [40] and Hartley [22].

C. Some elementary geometric lemmas.

LEMMA 2.9. Let $\Omega$ be a nonempty set of points of $P G(d, q), d \geqslant 2$, such that each line meets $\Omega$ in 0 or $k$ points, where $1<k<q+1$. Then

(i) $k \mid q$; and

(ii) If $d>2$, then $\Omega$ is the complement of a hyperplane.

Proof. If $W$ is an $i$-space, then $|W \cap \Omega|$ is 0 or

$$
n_{i}=1+(k-1)\left(q^{i}-1\right) /(q-1) .
$$

In order to prove (i), assume that $d=2$, pick a point $x \notin \Omega$, and note that there are $|\Omega| / k$ lines on $x$ meeting $\Omega$.

Now suppose that $d>2$. If every hyperplane met $\Omega$, then $\Omega$ and the hyperplanes would form a symmetric design with more blocks than points. Thus, some $d-2$-space $W$ misses $\Omega$. There are then $n_{d} / n_{d-1}$ hyperplanes containing $W$ and meeting $\Omega$. But 
$n_{d} / n_{d-1}$ is an integer only if $k=q$, in which case $n_{d}$ is the number of points outside of a hyperplane missing $\Omega$.

LEMMA 2.10. Let $V$ be a symplectic, unitary or orthogonal space with $n \geqslant 3$. Let $\Omega$ be a nonempty set of points such that every line meets $\Omega$ in 0 or q points. Then $\Omega$ is the set of all points not in some hyperplane.

Proof. If $\Omega^{\prime}$ denotes the set of points not in $\Omega$, then every line meeting $\Omega^{\prime}$ twice must have all its points in $\Omega^{\prime}$. Such a line exists: a plane $E$ meeting $\Omega$ has $|E \cap \Omega|=1+(q+1)(q-1)=q^{2}$, so that $E \cap \Omega$ is the complement of a line.

If $\left\langle\Omega^{\prime}\right\rangle \neq V$, then $\Omega$ contains all points not in $\left\langle\Omega^{\prime}\right\rangle$, and our assertion follows readily. If $\left\langle\Omega^{\prime}\right\rangle=V$, then $\Omega=\varnothing$ by Tits [58, (8.10)], which is absurd. (N.B.-Hypotheses (A) and (B) of [58, (8.10)] assert that the sesquilinear form on $V$ is trace-valued, which is always the case for finite fields.)

Combining (2.9) and (2.10) produces the following curious consequence.

COROllary 2.11. Let $\Omega$ be a nonempty set of points of a finite symplectic, unitary or orthogonal space $V$. Assume that $V$ contains totally isotropic (or totally singular) 4-spaces. If every line meets $\Omega$ in 0 or $k$ points, for some constant $k>1$, then $\Omega$ consists either of all points, or of all points off of some hyperplane.

A group $K \leqslant G$ is called $(i, j)$-transitive if it is transitive on the set of $(i, j)$-flags.

LEMMA 2.12. Suppose that $K \leqslant \Gamma(V)$ is transitive on the sets $\delta_{i}$ and $S_{j}$ of $i$-spaces and $j$-spaces. If $W \in \delta_{j}$, then all orbits of $K_{W}$ on $\delta_{i}$ have length divisible by $\left|\mathscr{S}_{i}\right| /\left(\left|\mathscr{S}_{i}\right|,\left|\mathscr{S}_{j}\right|\right)$. (In particular, if $i<j$ and $\left|\mathscr{S}_{i}\right| /\left(\left|\mathscr{S}_{i}\right|,\left|\delta_{j}\right|\right)$ is the number of $i$-spaces in a $j$-space, then $K$ is $(i, j)$-transitive. $)$

Proof. Let $U$ be any $i$-space. Then $\left\{\left(U^{k}, W^{k}\right) \mid k \in K\right\}$ has size $\left|\varsigma_{i}\right|\left|W^{K_{U}}\right|=\left|\delta_{j}\right|\left|U^{K_{w}}\right|$.

Lemma 2.13. Let $\Omega$ be a set of points, and $\delta_{k}, \delta_{k-1}$ collections of $k$-spaces (resp., $k-1$-spaces) contained in $\Omega$. Assume that every $k$-space on a member of $\mathcal{S}_{k-1}$ is in $\widehat{S}_{k}$. Then $\Omega$ consists of all points. (If $V$ has type $\Omega^{+}(2 k, q)$, modify the hypothesis so that all $k$-spaces considered have a fixed type.)

PRoOF. The elementary connectedness argument is omitted.

D. Cohomology. We will also require some results concerning the first cohomology groups of classical groups. These are collected in the next theorem.

THEOREM 2.14. (i) For a classical group $K=\operatorname{Chev}(V)$, set $d(K)=\operatorname{dim} H^{1}(K, V)$. Then $d(K)=0$, except in the following cases: $d(K)=1$ for $K=\Omega(3,5), \Omega(5,3)$, $S U(4,2), \Omega^{+}(6,2), \quad S U\left(2,2^{i}\right), \quad S p\left(2 n, 2^{i}\right)$, and $\Omega\left(2 n+1,2^{i}\right)$, except that $d(S U(2,2))=d(S p(2,2))=0$ and $d(\Omega(5,2))=2$; and $d\left(\Omega^{-}(4,3)\right)=2$.

(ii) $d(S L(V))=\operatorname{dim} H^{1}(S L(V), V)$ is 0 for $\operatorname{dim} V>2$, except that $d(S L(3,2))=1$.

(iii) $d(K)=0$ for each of (2.6 i, iii-vi) and (2.8 ii-iv), while $d(K)=1$ for (2.6 ii).

Proof. Parts (i) and (ii) are a summary of results of Higman [23], Pollatsek [47]-[49], Cline, Parshall and Scott [4], Jones [29], McLaughlin [39], Fischer [17, 
(16.1.10)], and Finkelstein [16, p. 82] (cf. Jones and Parshall [30]). The cases $S U(m, q)$ and $\Omega^{-}(2 m, q)$ with $q \leqslant 3$ (due to McLaughlin and to Pollatsek) are not in print, and will be dealt with here.

Our approach is very elementary, cocycles remaining invisible throughout. It is based primarily upon the following two elementary observations, which are simple consequences of the interpretation of first cohomology in terms of conjugacy classes of complements.

$(\alpha)$ Let $D \leqslant G L(V)$, and assume that $D$ has a normal $r$-subgroup $R \neq 1$ (where $r \neq p)$. If $W=C_{V}(R)$ is zero, or if $C_{D}(W)=O^{p}\left(C_{D}(W)\right)$ and $p \nmid\left|D^{W}\right|$, then $H^{1}(D, V)=0$.

( $\beta)$ If $K=\langle B, C\rangle$ with $H^{1}(B \cap C, V)=H^{1}(B, V)=H^{1}(C, V)=0$ and $C_{V}(B \cap C)=0$, then $H^{1}(K, V)=0$.

Proof of $(\alpha)$. If $D V=D_{1} V \geqslant R V$ and $D_{1} \cap V=1$, we may assume that $D \cap D_{1} \geqslant R$. Then $D W=N_{D V}(R)=D_{1} W$, and $D=D_{1}$ if $|W|=1$. If $|W|>1$ then $C_{D}(W)=O^{p}\left(C_{D W}(W)\right)=C_{D_{1}}(W)$, so that $\left(D / C_{D}(W)\right) W=\left(D_{1} / C_{D}(W)\right) W$. Thus, $D^{W}=D / C_{D}(W)$ and $D_{1} / C_{D}(W)$ are conjugate under $W$.

Proof of $(\beta)$. Let $V$ be a hyperplane of a space $U$, and embed $K$ into $G L(U)$ so as to fix a vector $u \notin V$. Set $T=O_{p}\left(G L(U)_{V}\right)$. Then $D$ acts on $T$ as it does on $V$. Let $K T=K_{1} T$ with $K_{1} \cap T=1$. Let $B_{1}, C_{1} \leqslant K_{1}$ correspond to $B, C$. By hypothesis, there is a unique 1-space $\left\langle u_{1}\right\rangle$ of $U$ centralized by $B_{1} \cap C_{1}$. Using $T$, we may assume that $u_{1}=u$. There is also a unique 1-space centralized by $B_{1}$ (resp., $C_{1}$ ). Thus, $\left\langle B_{1}, C_{1}\right\rangle=K_{1}$ centralizes $u$, so $K_{1}=C_{K T}(u)=K$.

Remarks. Assertion $(\beta)$ is due to Alperin and Gorenstein [1], where an entirely different proof is given. (Their more general result is also immediate, using the above argument.) Yet another elementary proof can be obtained by translating the above one into statements concerning cocycles. Note that $U$ and $T$ were introduced in order to avoid using the elementary fact that $H^{1}(K, V) \cong \operatorname{Ext}_{G F(q) K}(G F(q), V)$.

We will use $(\alpha)$ and $(\beta)$ in several examples, primarily those not already in print. We may assume that $(\alpha)$ does not apply with $D=K$ (and in particular that $Z(K)=1)$. This handles (2.6 iii-vi) and (2.8 ii, iv). Of course, in situations in which $d(K)>0$, ad hoc methods will be needed. Note that $d(K)$ can be proved to be nonzero by exhibiting an indecomposable $K$-module containing the $K$-submodule $V$ as a hyperplane.

EXAMPLE 1. $P S L(2,7)<S U(3,3)$. Use $B \cong C \cong S_{4}$ with $B \cap C=D_{8}$ in $(\beta)$.

Example 2. $K=S U(m, q), m \geqslant 3$, excluding $S U(4,2)$ (McLaughlin [39]). Let $b$ and $c$ be perpendicular 1-spaces, and set $B=K_{b}$ and $C=K_{c}$. Note that $1 \neq Z(B)<C$ and $C_{V}(Z(B) Z(C))=0$, so $H^{1}(B \cap C, V)=0$ by $(\alpha)$. Since $B \neq S U(3,2)$, either $C_{V}(Z(B))=0$ or $C_{B}(b)=O^{p}\left(C_{B}(b)\right)$. Thus, $H^{1}(B, V)=0$ by $(\alpha)$, and $(\beta)$ applies.

Now consider the case $K=S U(4,2) \cong \Omega(5,3)$. Let $B$ and $C$ be maximal parabolic subgroups of $\Omega(5,3)$ such that $K=\langle B, C\rangle, B \cap C$ contains a Sylow 3-subgroup of $K$, and $B / O_{3}(B) \cong S L(2,3)$. Then $C$ acts monomially on $V$, so that $H^{1}(C, V)=0$ by $(\alpha)$. Suppose that $K V=K_{1} V$ with $K_{1} \cap V=1$. We may assume that $K \cap K_{1} \geqslant C$. If $R=O_{3}(B)$, then $K_{1}=\left\langle C, N_{K_{1}}(R)\right\rangle$. But $N_{K V}(R) / R$ has at most 4 subgroups 
$\cong S L(2,3)$, since $B=N_{K}(R)$ is the stabilizer of a nonsingular 1-space of $V$. Thus, $d(K) \leqslant 1$. That $d(K)=1$ is due to Fischer [17, (16.1.10)], and is discussed in [33, $\S 2]$ and Case 4 of the proof of (2.16).

Example 3. $K=\Omega(3, q)$. If $q>5$ and $q$ is odd, use $B \cong A_{4}$ and $C$ dihedral of order $q \pm 1$, with $|B \cap C|=4$. If $q=3,(\alpha)$ applies. Higman [23] and Pollatsek [47] proved that $d(\Omega(3, q))=1$ for $q$ even.

Consider $K=\Omega(3,5)$. First use the standard permutation module for $A_{5}$ over $G F(5)$ in order to find that $d(K) \neq 0$. Then suppose that $K V=K_{1} V$ with $K_{1} \cap V=1$. By $(\alpha)$, we may assume that $K \cap K_{1} \geqslant B \cong A_{4}$. Let $T \in \operatorname{Syl}_{3}(B)$, and note that $K_{1}=\left\langle B, N_{K_{1}}(T)\right\rangle$. Since $\left|C_{V}(T)\right|=5, N_{K V}(T)$ has exactly 5 subgroups $S_{3}$, so there are at most 5 choices for $N_{K_{1}}(T)$. Thus, $d(K) \leqslant 1$.

EXAMPLE 4. $K=\Omega^{-}(4, q)$. Set $B=K_{b}$ for a nonsingular l-space $b$. If $q$ is odd and $q \geqslant 5$, let $C$ be a dihedral group of order $q^{2}-1$, chosen so that $|B \cap C|=2(q \pm 1) \equiv 0(\bmod 8)$. Then $K=\langle B, C\rangle($ since $q>5)$, and $H^{1}(B, V)=0$ by Example 3, while $H^{1}(B \cap C, V)=0$ by $(\alpha)$. Also, $B \cap C$ is not inside $C_{K}(b)=\Omega(3, q)$, and hence $C_{V}(B \cap C)=0$. Hence, $(\beta)$ applies.

If $q$ is even and $q>2$, then $\operatorname{dim} H^{1}(B, V / b)=1$ (Example 3), while $B$ acts indecomposably on $V$. Thus, $H^{1}(B, V)=0$. Let $K V=K_{1} V$ with $K_{1} \cap V=1$. We may assume that $K \cap K_{1} \geqslant B$. Let $A$ be a dihedral subgroup of $B$ of order 2( $\left.q-1\right)$. Then $K_{1}=\left\langle B, A, C_{K_{1}}\left(A^{\prime}\right)\right\rangle$, where $\left\langle A, C_{K_{1}}\left(A^{\prime}\right)\right\rangle$ is dihedral of order $2\left(q^{2}-1\right)$. Here, $\left\langle A, C_{K V}\left(A^{\prime}\right)\right\rangle=b \times\left\langle A, C_{K}\left(A^{\prime}\right)\right\rangle$ has exactly $q$ such dihedral subgroups, and these are conjugate under $b$. Since $b$ centralizes $B$, it follows that $K$ and $K_{1}$ are also conjugate under $b$. Thus, $d(K)=0$ (Jones [29]).

Next, consider the case $q=3$. If $K V=K_{1} V$ with $K_{1} \cap V=1$, we may assume that $K \cap K_{1} \geqslant D$ with $|D|=10$. Let $t$ be an involution of $D$. Then $K_{1}=\langle D, g\rangle$ with $g^{2}=t$. But $\left|t^{V}\right|=9$, so that $C_{K V}(t)$ has only 9 subgroups of order 4 . Thus, at most 9 classes of complements exist. Using generators and relations, Finkelstein [16, p. 82] showed that 9 classes actually occur. An alternative procedure is as follows. Let $W$ be the 6-dimensional permutation module for $S_{6}$ over $G F(3)$, equipped with the usual inner product. If $w$ is its $S_{6}$-invariant 1-space, then $S_{6}$ has a unique orbit of length 6 on $W / w$. Thus, Aut $S_{6}$ cannot act on $W / w$, but it certainly acts on $H^{1}\left(A_{6}, w^{\perp} / w\right)$. Consequently, $\operatorname{dim} H^{1}\left(\Omega^{-}(4,3), w^{\perp} / w\right)>1$.

Finally, consider $K=\Omega^{-}(4,2)$. Let $K V=K_{1} V$ with $K_{1} \cap V=1$. We may assume that $K \cap K_{1}$ contains $\langle s, t\rangle \cong D_{10}$ with $s^{2}=t^{2}=1$. Then $K=\langle r, s, t\rangle$ for a uniquely determined involution $r \in C_{K}(t)$ such that $|r s|=3$. Thus, $K_{1}=\left\langle r_{1}, s, t\right\rangle$ with $r_{1}^{2}=1, r_{1} \in C_{K}(t),\left|r_{1} s\right|=3$, and $r r_{1} \in V$. Since $\left|r r_{1}\right|=2, r r_{1} \in C_{V}(\langle r, t\rangle)$. Suppose that $r \neq r_{1}$. Then $v=r r_{1}$ is uniquely determined $\left(\right.$ since $\left.\operatorname{dim} C_{V}(\langle r, t\rangle)=1\right)$, and is singular. But $\left(s r_{1}\right)^{3}=(s r v)^{3}=v+v^{s r}+v^{(s r)^{2}}$. Since $|s r|=3$ and $s r$ moves $v$, the singular vectors $v, v^{s r}$ and $v^{(s r)^{2}}$ are linearly independent. Thus, $\left|s r_{1}\right| \neq 3$. This contradiction shows that $K_{1}=K$, as desired.

Example 5. $K=\Omega^{+}(4, q)$. If $q$ is odd, then $-1 \in Z(K)$ and $(\alpha)$ applies. If $q=2$, $(\alpha)$ also applies. If $q$ is even and $q>2$, the corresponding argument in Example 4 applies. 
Example 6. $K=\Omega^{-}(2 m, q), m \geqslant 3$. Let $B$ and $C$ be the stabilizers of two perpendicular anisotropic 2 -spaces. By $(\alpha)$, Example 4, or induction, $(\beta)$ applies to $\langle B, C\rangle$. Here, $\langle B, C\rangle=K$ except when $K=\Omega^{-}(6,3)$ or $\Omega^{-}(6,2)$. Note that $-1 \in \Omega^{-}(6,3)$ (since $-1 \in \Omega^{-}(2,3)$ ), so that $(\alpha)$ applies here.

Finally, if $K=\Omega^{-}(6,2) \cong \Omega(5,3)$, let $B_{0}$ and $C_{0}$ be maximal parabolic subgroups of $\Omega(5,3)$ containing a Sylow 3-subgroup of $\Omega(5,3)$. Then $(\beta)$ applies to $\left\langle B_{0}, C_{0}\right\rangle=K$, by $(\alpha)$.

Example 7. $K$ is $A_{6}$, inside $S p(4,2)$ or $\Omega(5,2)$. Using the standard 6-dimensional permutation module for $K$ over $G F(2)$, we find that $d(K) \neq 0$.

Let $K V=K_{1} V$ with $K_{1} \cap V=1$. By Example 4, we may assume that $K \cap K_{1}$ contains $A_{5} \cong \Omega^{-}(4,2)$. Let $T \in S y l_{3}(B)$. Then $K_{1}=\left\langle B, N_{K_{1}}(T)\right\rangle$. However, $N_{K V}(T)$ has exactly two Sylow 3 -subgroups containing $N_{B}(T)$. Thus, $d(K) \leqslant 1$, as required.

EXAMPLE 8. $K$ is $A_{7}$ or $S_{7}$, inside $O^{+}(V)=O^{+}(6,2)$. Here, $d(K)=0$ (proved for $S_{7}$ in Pollatsek [47]). For, let $K V=K_{1} V$ with $K_{1} \cap V=1$. We may assume that $K \cap K_{1}$ contains a Frobenius group $B$ of order 21. Let $T \in S_{y l} B$. Then $K_{1}=\left\langle B, C_{K_{1}}(T)\right\rangle$. But $C_{K V}(T)=C_{K}(T) \times C_{V}(T)$. Thus, $C_{K_{1}}(T)=C_{K}(T)$ and $K_{1}=K$.

Remark. We have concentrated on low dimensional examples. The higher dimensional ones can be readily handled inductively, as in Examples 2 and 6.

Proposition 2.15. Let $H \leqslant \Gamma L(W)=\Gamma L(n, q), n \geqslant 3$. Suppose that $H$ is not flag-transitive, but that $H_{x}$ is point-transitive on $W / x$ for some point $x$. Then one of the following holds:

(i) $H$ fixes a point or hyperplane; or

(ii) $H$ is $3 \cdot A_{6}$ or $3 \cdot S_{6}$ inside $\Gamma L(3,4)$, fixing a hyperoval.

Proof. Clearly, $\Omega=x^{H}$ does not consist of all points, and we may assume that $|\Omega|>1$. If $n=3$, use Mitchell [40] and Hartley [22]. If $n \geqslant 4$, then (2.9) yields (i).

The next result is similar, but more interesting.

THEOREM 2.16. Let $K \leqslant \Gamma(V)$, where $V$ has rank $n \geqslant 3$ but does not have type $\Omega^{+}(6, q)$. Let $x$ be a point moved by $K$, and assume that $K_{x}$ is flag-transitive on $x^{\perp} / x$. Then one of the following holds.

(i) $K$ fixes a nondegenerate hyperplane or a nonsingular 2-space.

(ii) $K \unrhd 3 \cdot P S U(4,3)$, inside $\Gamma U(6,2)$.

(iii) $K$ is $A_{8}$ or $S_{8}$ inside $O^{+}(8,2)$, and fixes a point other than $x$.

(iv) $K$ is $A_{9}$ or $S_{9}$ inside $O^{+}(8,2)$.

(v) $K \unrhd \Omega^{-}(6,2)$, inside $\Gamma O(7,3)$, where $K$ fixes a point other than $x$.

(vi) $K \unrhd S p(6,2)$, inside $\Gamma O(7,3)$.

REMARK. Some interpretation is required for (i) when $V$ is of type $\operatorname{Sp}\left(2 n, 2^{i}\right)$ or $\Omega\left(2 n+1,2^{i}\right)$. Namely, fixing a nonsingular 2-space of the former must be regarded as essentially the same as fixing a nondegenerate 3 -space of the latter, while fixing an 
orthogonal subgeometry $O^{+}\left(2 n, 2^{i}\right)$ of the former must be identified with fixing a nonsingular hyperplane of the latter.

Proof. We may assume that (i) does not hold and that $K$ contains no long root elements ((2.3) and [33]). Let $Q=O_{p}\left(\Gamma(V)_{x}\right)$ and let $Q_{1}$ be the group of transvections in $Q$ (as in (2.1)). Then $K \cap Q_{1}=1$.

In fact, $K \cap Q=1$. For otherwise, $Q=(K \cap Q) Q_{1}$ by (2.1) and the irreducibility of $K_{x}$ on $x^{\perp} / x$. If $Q^{\prime} \neq 1$, then $Q_{1}=Q^{\prime}=\Phi(Q)$ implies that $K_{x} \cap Q=Q>Q_{1}$. Thus, $Q^{\prime}=1$, and similarly, $Q_{1} \neq 1$, so we are in the case $\operatorname{Sp}(2 m, q)$ with $q$ even. But here, $K_{x}$ acts indecomposably on $Q$ (by (2.6)), so that again $K_{x} \cap Q=Q$.

Clearly, $K_{x}^{x^{\perp} / x}$ is as in (2.6) or contains $\operatorname{Chev}\left(x^{\perp} / x\right)$. In the latter case, $K_{x}$ has a normal subgroup $D \cong \operatorname{Chev}\left(x^{\perp} / x\right)$. If $H^{1}\left(D, x^{\perp} / x\right)=0$, then $H^{1}\left(D, Q / Q_{1}\right)=0$ by (2.1), so $D Q_{1}$ is unique up to conjugacy in $\operatorname{Chev}(V)$, and hence so is $\left(D Q_{1}\right)^{\prime}=D$; in this case, $K_{x}$ has a subgroup which centralizes a nonsingular 2-space containing $x$ and induces $\operatorname{Chev}\left(x^{\perp} / x\right)$ on $x^{\perp} / x$, so that $K_{x}$ contains long root groups. Consequently, we are only left with the possibilities that either $H^{1}\left(D, x^{\perp} / x\right) \neq 0$ or that $K_{x}^{x^{\perp} / x}$ is one of the exceptional cases occurring in (2.6). By (2.14), $V$ must have type $S p(2 m, q)$ with $q$ even, $S p(6,3), \Omega(7,3), \Omega^{+}(8,2), S U(6,2), \Omega^{-}(8,3)$ or $S U(6,3)$.

We will consider these possibilities separately. Set $\Omega=x^{K}$.

Each line meets $\Omega$ in 0 or $k$ points, for some constant $k$. If $E$ is a plane meeting $\Omega$ more than once, then (2.9) applies to $K_{E}^{E}$. Thus, by (2.10), if $k>1$ then $V$ has type $S U(6,2)$ and $E \cap \Omega$ is a hyperoval of $E$ (which is precisely what happens in (ii)).

Case 1. $K \leqslant S p(2 n, 2), n \geqslant 3, q$ even, excluding $S p(6,2)$. By (2.14), $\operatorname{dim} H^{1}(D, Q)=1$. By [33, $\$ 2$, Example RL1], each complement to $Q$ in $D Q$ contains long root elements.

Case 2. $K_{x}$ is $A_{7}, S_{7}, A_{8}$ or $S_{8}$, inside $O^{+}(8,2)$. Here, $k=1$, so that $x^{\perp} \cap \Omega=\{x\}$. Let $K_{x} \geqslant A=A_{7}$. According to (2.14 iii), there is essentially just one possibility for $A$. Thus, there is a basis $\left\{e_{i}\right\}$ of pairwise nonperpendicular nonsingular vectors of $V$ such that (identifying each point with the corresponding vector) $x=\Sigma e_{i}$ and $A$ permutes the basis, fixing $e_{8}$. The point-orbits of $A$ have lengths $1,1,7,21,35,35$, 35 . Since $k=1$ it follows easily that $|\Omega| \leqslant 9$. Then $\Omega$ is preserved by the transvection $t$ with direction $e_{1}+e_{2}$, and $\langle K, t\rangle$ must be $S_{8}$ or $S_{9}$. Thus, (iii) or (iv) holds.

Case 3. $K_{x}$ is $A_{6}$ or $S_{6}$, inside $O(7,2)$. Let $K_{x} \geqslant A=A_{6}$. Once again, $x^{\perp} \cap \Omega=\{x\}$. By (2.14 iii), there are two possibilities for the action of $A$ on $V$.

Suppose first that $C_{V}(A)$ is a nondegenerate 3-space. Then the point-orbits of $A$ have lengths $1,1,1,15,15,15,15$ (as is most easily seen by regarding $A$ as being inside $S p(6,2)$ ). Since each nontrivial orbit contains a pair of perpendicular points, $|\Omega| \leqslant 3$. Thus, (i) holds when $V$ is replaced by $V / \operatorname{rad} V$.

Thus, $\operatorname{dim} C_{V}(A)=2$ and $V$ has a basis $\left\{e_{i}\right\}$ of pairwise nonperpendicular singular vectors which $A$ permutes while fixing $x=e_{7}$. This time, $x^{\perp} \cap \Omega=\{x\}$ implies that $|\Omega|=7$ and that $K$ is $A_{7}$ or $S_{7}$. But $K$ then fixes the nonsingular hyperplane $\left\{\sum \alpha_{i} e_{i} \mid \sum \alpha_{i}=0\right\}$.

Case 4. $K<\Gamma U(6,2)$. Here, $K_{x} \unrhd H=S U(4,2)$, where $H$ does not fix a nonsingular 2-space. By Fischer [17, (16.1.10)], $H$ is uniquely determined up to 
conjugacy in $\Gamma U(6,2)$ (compare (2.14 i)), and there is a transvection $t \in S U(6,2)_{x}$ normalizing but not centralizing $H$. Set $z=[V, t]$ and $K^{+}=\left\langle H, K_{z}, t\right\rangle \unrhd\left\langle H, K_{z}\right\rangle$.

If $K^{+}$is irreducible on $V$, then [33] applies. In this case, $K^{+} \cap S U(6,2)=$ $3 \cdot \operatorname{PSU}(4,3) \cdot 2$, so that $K \geqslant 3 \cdot \operatorname{PSU}(4,3)$. The latter group has only two orbits on points: one of length 126 containing $z$, and one of length 567 (Fischer [17, (16.1.16)]). Consequently $(K\langle t\rangle) \cap S U(V)=3 \cdot P S U(4,3) \cdot 2$, and (2.16 ii) holds.

If $K^{+}$is reducible on $V$, then $K^{+}$must fix $x$ (since $H$ fixes only two proper subspaces of $V$, namely, $x$ and $x^{\perp}$ ). Thus, $K_{z} \leqslant K_{x}$. Let $E$ be any plane containing $\langle x, z\rangle$. Then $K_{E}^{E}$ contains $A_{6}$, so that $K_{z}$ cannot fix $x$, and this situation cannot occur.

Case 5. $K<\Gamma O^{-}(8,3)$. Here $K_{x} \triangleright H=2 \cdot \operatorname{PSL}(3,4)$, so that $\left|Z\left(K_{x}\right)\right|=2$. Then $K_{x}$ fixes a hyperbolic line containing $x$, and hence also a second point $y$. Note that $H$ is transitive on each class of nonsingular points in $\langle x, y\rangle^{\perp}$. (For, $\Omega^{-}(6,3)$ has rank 3 on each class of nonsingular points, as well as on the conjugates of $H$, and it is easy to check the degrees of the irreducible constituents of the permutation characters.) Thus, each $H$-orbit of points not in $\langle x, y\rangle \cup\langle x, y\rangle^{\perp}$ has length 56 or 126. It is now easy to check that $x^{\perp} \cap \Omega=\{x\}$ forces $\Omega$ to be $\{x, y\}$, so that (i) holds.

Case 6. $K<\Gamma U(6,3)$. Here, $K_{x} \unrhd H=4 \cdot P S L(3,4)$, and $K_{x}$ fixes a hyperbolic line $T$ containing $x$. Moreover, $H$ centralizes $T$ and is point-transitive on $T^{\perp}$. Since $K$ moves $T, \Omega$ must contain a point of the form $\langle u+v\rangle$ with $u \in T, v \in T^{\perp}$ and $(u, u)+(v, v)=0$. Let $\langle a\rangle$ be a point in $T^{\perp} \cap v^{\perp}$. Then a Sylow 3-subgroup $P$ of $H_{a}$ fixes $\langle a, v\rangle$. Also, $P$ moves $v$ (as otherwise, $P$ would act on $T^{\perp} \cap v^{\perp}$, and hence would have an element inducing a transvection on $V$ ). Thus, $\Omega$ contains $\langle u+v+\alpha a\rangle$ for some scalar $\alpha \neq 0$. Since $(u+v, u+v+\alpha a)=0$, this contradicts the fact that $k=1$.

Case 7. $K<\Gamma S p(6,3)$. Here, $K_{x}>H$ with $O_{2}(H)$ extraspecial of order $2^{5}$ and $\left|H / O_{2}(H)\right|=5$. Once again, $K_{x}$ fixes a nonsingular 2-space $T$ on $x$. Then $H$ centralizes $T$ and is transitive on $T^{\perp}$. Thus, since $k=1$, (i) holds.

Our last case is the most interesting one, since it leads to $(2.16 \mathrm{v}$, vi).

Case 8. $K<\Gamma O(7,3)$. Here, $K_{x}>H$ with $A=O_{2}(H)$ elementary abelian of order $2^{4}$ and $|H / A|=5$. Then $H$ fixes a point $y \neq x$. There is an orthogonal basis $\left\{b_{i}\right\}$ of $\langle x, y\rangle^{\perp}$ such that $H$ permutes the 1-spaces $\left\langle b_{i}\right\rangle$. We may assume that $\left(b_{i}, b_{i}\right)=1$ for all $i$. Fix $d_{j} \in\langle x, y\rangle$ with $\left(d_{j}, d_{j}\right)=j$ for $j= \pm 1$.

Recall that $x^{\perp} \cap \Omega=\{x\}$. Using $\left\{b_{i}\right\}$, it is easy to check that $H$ has only 3 point-orbits outside of $\langle x, y\rangle$ which might belong to $\Omega$, namely

$$
B=\left\{\left\langle d_{-1} \pm b_{i}\right\rangle \mid 1 \leqslant i \leqslant 5\right\} \quad \text { and } \quad F_{\mu}=\left\{\left\langle d_{1}+\sum_{1}^{5} \varepsilon_{i} b_{i}\right\rangle \mid \prod_{1}^{5} \varepsilon_{i}=\mu\right\} \text { for } \mu= \pm 1 \text {. }
$$

Here, $|B|=10$ while $\left|F_{\mu}\right|=16$. Thus, $|\Omega|$ is $11,12,17,18,27$ or 28 (since $\left(d_{1}+\Sigma_{1}^{5} b_{i}, d_{1}+b_{1}+b_{2}-b_{3}-b_{4}-b_{5}\right)=0$ and $\left.x^{\perp} \cap \Omega=\{x\}\right)$. The third of these fails to divide $|\Gamma O(7,3)|$, while the first leads to a nonexistent group of degree 11. 
Suppose that $|\Omega|=12$. Then $K_{x}$ cannot induce $\Omega(5,3)$ on $x^{\perp} / x$, so that $K_{x} \triangleright A$. Consequently, $K$ acts imprimitively on $\Omega$, with 6 blocks of size 2 which $K$ permutes 2 -transitively. However, the 2 -space spanned by a block is $\langle x, y\rangle$ or $\left\langle d_{-1}+b_{i}, d_{-1}-b_{i}\right\rangle$, and hence contains $d_{-1}$. Thus, $K$ fixes $\left\langle d_{-1}\right\rangle$, and (i) holds. (N.B.-This case actually occurs.)

Assume that $|\Omega|=18$. Once again $K_{x} \triangleright A$ and $K$ acts imprimitively on $\Omega$. However, $K_{x}$ acts primitively on $F_{\mu}$, so that this situation cannot occur.

Consequently, $|\Omega|$ is 27 or 28 . Note that, in either case, $\Omega$ is uniquely determined (as $\{x\} \cup B \cup F_{1}$ or $\{x, y\} \cup B \cup F_{1}$ ) up to $\Gamma O(7,3)$-equivalence. Thus, it suffices to show that $K^{*}=\Gamma O(7,3)_{\Omega}$ has the required transitivity properties, and then to study subgroups of $K^{*}$ containing $H$. For this we will require a digression involving root systems mod 3.

Digression. In order to examine the embedding of the Weyl group $W=W\left(E_{7}\right)$ of type $E_{7}$ into $O(7,3)$, we will consider the embedding of the Weyl group $W\left(E_{8}\right)$ into $O^{+}(8,3)$. The latter embedding will also arise in later sections.

Let $\left\{e_{i}\right\}$ be an orthonormal basis for an $O^{+}(8,3)$-space. Form the vectors $\varepsilon_{i} e_{i}+\varepsilon_{j} e_{j}(i \neq j)$ and $\Sigma_{1}^{8} \varepsilon_{i} e_{i}$ with $\Pi_{1}^{8} \varepsilon_{i}=-1$, where each $\varepsilon_{i}$ is \pm 1 . Then $W\left(E_{8}\right)$ is generated by the reflections in the hyperplanes perpendicular to the above "root" vectors (Carter [3, p. 48]).

Set $\rho=e_{7}-e_{8}$ and $u=e_{6}+e_{7}+e_{8}=-\rho+\left(e_{6}-e_{7}\right)$, and write $W=W\left(E_{8}\right)_{\rho}$. Then $W_{u}=W\left(E_{6}\right)$, so $\left(W_{u}\right)^{\prime} \cong \Omega(5,3)$. Moreover, $\left(W_{u}\right)^{\prime}$ acts faithfully on $u^{\perp} /\langle u\rangle$ as $\Omega(5,3)$. In particular, $W^{\prime}$ behaves as in (vi), with $x=\langle u\rangle$ and $\left|\langle u\rangle^{W}\right|=\left|W: W_{\langle u\rangle}\right|=28$.

Now consider $W_{u}$. This group acts on the set of 27 roots $\alpha$ such that $\alpha \in \rho^{\perp}$ and $\alpha+\left(e_{6}-e_{7}\right)$ is a root. (These roots $\alpha$ are $e_{7}+e_{8}, \pm e_{i}-e_{6}$ with $1 \leqslant i \leqslant 5$, and $\Sigma_{1}^{5} \varepsilon_{i} e_{i}+e_{6}-e_{7}-e_{8}$ with $\prod_{1}^{5} \varepsilon_{i}=-1$.) Then $W_{u}$ also acts on the corresponding 27 points $\langle\alpha+u\rangle$. One of these is $\left\langle u^{\prime}\right\rangle$ with $u^{\prime}=e_{6}-e_{7}-e_{8}$, and $W_{u u^{\prime}}=W\left(D_{5}\right)$. Consequently, $(2.16 \mathrm{v})$ holds when $K=\left(W_{u}\right)^{\prime}$ and $x=\left\langle u^{\prime}\right\rangle$. (N.B.- The correspondence with our previous notation is as follows: $x=\left\langle u^{\prime}\right\rangle, y=\langle u\rangle$, $d_{j}=-j u^{\prime}-u, b_{i}=e_{i}$.)

We now return to Case 8 . Which subgroups $K$ of $K^{*}=\Gamma O(7,3)_{\Omega}$ behave as required in (2.16)? We may assume that $|\Omega|=28$. Then $W \leqslant K^{*}<\Gamma O(7,3)$. By Fischer [17, (15.3.16)], $K^{*}=\langle-1\rangle \times W$. First suppose that

$$
K \leqslant K_{y}^{*} \cong\langle-1\rangle \times O^{-}(6,2) .
$$

Two suitable $K_{y}^{*}$-conjugates of $A=O_{2}(H)$ generate $\Omega^{-}(6,2)$, so that $K$ is as in $(2.16 \mathrm{v})$. Now suppose that $K$ is transitive on $\Omega$. If $K_{x} \geqslant \Omega(5,3)$, then (2.16 vi) certainly holds. Suppose that $A \triangleright K_{x}$. Then $K$ acts imprimitively on $\Omega$, with 14 blocks of size 2 . Since an element of $K^{*}$ of order 5 fixes only 3 members of $\Omega$, this is impossible.

This completes the proof of (2.16).

REMARK 2.17. We will need further information concerning the embedding of $W\left(E_{8}\right)$ into $O^{+}(8,3)$, whose study was begun in Case 8 of (2.16). The vectors $e_{i}, \rho$ and $u$ were defined there. Changing notation somewhat, we will now write $x=\langle u\rangle$ and $K=W\left(E_{8}\right)$. 
Note that $\langle u, \rho\rangle$ contains all root vectors $\beta$ such that $\beta-u$ is also a root. Thus, $K_{x}=N_{K}(\langle u, \rho\rangle)($ since $x=\operatorname{rad}\langle u, \rho\rangle)$, where $K_{x}=K_{u} \times\langle-1\rangle$. Recall that $K_{u \rho}$ is $W\left(E_{6}\right)$. Moreover, $K_{u}=K_{u \rho}\left\langle r_{\rho}, r_{u+\rho}\right\rangle$ where $r_{\rho}$ is the reflection with axis $\rho^{\perp}$. Note that $\left\langle r_{\rho}, r_{u+\rho}\right\rangle \cong S_{3}$, and it contains the 3 -cycle $t=(6,7,8)$ acting on $\left\{e_{i}\right\}$. Here, $t$ centralizes both $K_{u \rho}$ and $x^{\perp} / x$.

The points of our $O^{+}(8,3)$ have the shape $\left( \pm 1^{3} 0^{5}\right)$ or $\left( \pm 1^{6} 0^{2}\right)$. It follows that $K$ is transitive on them. Also, $K_{x}$ induces $O(5,3)$ on $x^{\perp} / x$, so that $K$ has 2 orbits of $(1,2)$-flags and is transitive on $(1,4)$-flags. Then $K^{\prime}=\Omega^{+}(8,2)$ is transitive on the (1,4)-flags of each type. Moreover, if $F$ is a 4 -space then $W_{F} \cong 3 \cdot \operatorname{Sp}(4,3)$; this is easily proved directly, but also follows from the fact that Aut $\Omega^{+}(8,2)<$ Aut $P \Omega^{+}(8,3)$.

E. Primitive divisors. If $q>1$, a primitive divisor of $q^{k}-1$ is a prime $r$ dividing $q^{k}-1$, but not dividing $q^{i}-1$ for $1 \leqslant i<k$. (Note that $k \mid r-1$.) For convenience, a primitive divisor of $q^{k}+1$ is defined to be the same as a primitive divisor of $q^{2 k}-1$. The following number-theoretic fact will be crucial in $\S 6$.

LEMMA 2.18. $q^{k}-1$ has a primitive divisor, with the following exceptions:

(i) $q=2, k=6$; or

(ii) $q$ is a Mersenne prime, and $k=2$.

Proof. Zsigmondy [67].

The following simple result will be used often, frequently without reference.

LEMMA 2.19. Let $r$ be a primitive divisor of $q^{k}-1$, and $R$ a nontrivial $r$-subgroup of $\Gamma(V)$. Then

(i) $V=C_{V}(R) \perp[V, R]$, and

(ii) each irreducible constituent of $[V, R]$ has dimension $k$.

3. Characters of the Weyl groups. Let $B_{n}=Z_{2}$ wr $S_{n}$ be the $n$-dimensional hyperoctahedral group, viewed as the group of all "signed" permutations of $\Omega=\{ \pm 1, \ldots, \pm n\}$. Then $B_{n}$ is generated by $\{(i, i+1)(-i,-i-1)$; $(n,-n) \mid i=1, \ldots, n-1\} \subseteq S_{\Omega}$. The groups $B_{n}$ and $D_{n}=B_{n} \cap A_{\Omega}$ are the Weyl groups of classical groups, and hence the proof of our main theorem requires explicit information about certain of their permutation characters.

The purpose of this section is to describe how the appropriate permutation characters decompose as sums of irreducible characters. The authors acknowledge the generous help of Jan Saxl in the development of Table II.

A partition $\lambda$ of the natural number $s$, written $\lambda+s$, is a nonincreasing sequence of nonnegative integers $\lambda_{1}, \ldots, \lambda_{s}$ such that $\Sigma \lambda_{i}=s$. The length $k$ of such a partition is defined by $\lambda_{k+1}=0 \neq \lambda_{k}$. We say a subset $X$ of $\Omega$ is admissible when $x \in X$ if and only if $-x \in X$. In case $X \subseteq \Omega$ is admissible we define $X^{+}=X \cap\{1,2, \ldots, n\}$ and $X^{-}=X \cap\{-1,-2, \ldots,-n\}$, and consider two groups of permutations of $\Omega$ :

$$
A_{X}=\left\langle\left(x, x^{\prime}\right)\left(-x,-x^{\prime}\right) \mid x, x^{\prime} \in X^{+}\right\rangle
$$

and

$$
B_{X}=\left\langle A_{X},(x,-x) \mid x \in X^{+}\right\rangle \text {. }
$$


Observe that $|X|=2 k$ if $X$ is admissible and that $A_{X}$ is isomorphic to the symmetric group $S_{k}$ while $B_{X}$ is isomorphic to the hyperoctahedral group $B_{k}$.

Let $(\lambda ; u)$ be a pair of partitions where $\lambda \vdash s$ has length $l$ and $\mu \vdash n-s$ has length $m$. Partition $\Omega$ into admissible sets $L_{1}, \ldots, L_{l}, M_{1}, \ldots, M_{m}$ so that $\left|L_{i}\right|=2 \lambda_{i}$ and $\left|M_{j}\right|=2 \mu_{j}$ for $1 \leqslant i \leqslant l, 1 \leqslant j \leqslant m$, and consider the Weyl subgroup (notation of Mayer [35])

$$
W(\lambda ; \mu)=\left\langle A_{L_{1}}, B_{M_{j}} \mid i=1, \ldots, l ; j=1, \ldots, m\right\rangle \leqslant B_{n} .
$$

It is easy to see that the conjugacy class of $W(\lambda ; \mu)$ is uniquely determined by $(\lambda ; \mu)$.

The Weyl subgroups of $B_{n}$ play a very important role in the character theory of $B_{n}$ that is quite analogous to the role played by the Young subgroups $W(\lambda ; \varphi)$ in the character theory of the symmetric groups (in the notation of Kerber [34]). In spite of this we are most interested in the parabolic Weyl subgroups, i.e., those that correspond to parabolic subgroups of a group with a $(B, N)$-pair of type $B_{n}$. These are the Weyl subgroups $W(\lambda ; \mu)$ where $l \leqslant 1$.

Let $1(\lambda ; \mu)$ denote the character of $B_{n}$ obtained by inducing the trivial character of $W(\lambda ; \mu)$ to $B_{n}$, and let $\varepsilon(\lambda ; \mu)$ denote the character of $B_{n}$ obtained by inducing the alternating character $\varepsilon$ of $W(\lambda ; \mu)$ to $B_{n}$. (Recall that $\varepsilon(g)=-1$ if $g$ is an odd permutation in $S_{\Omega}$ and $\varepsilon(g)=1$ if $g$ is an even permutation in $S_{\Omega}$.)

Let $C(l, m)$ denote the $2 l+m$ by $2 l+m$ matrix having block diagonal form:

$$
C(l, m)=\operatorname{diag}\{\underbrace{\left(\begin{array}{ll}
0 & 1 \\
1 & 0
\end{array}\right),\left(\begin{array}{ll}
0 & 1 \\
1 & 0
\end{array}\right), \ldots,\left(\begin{array}{ll}
0 & 1 \\
1 & 0
\end{array}\right)}_{l}, \underbrace{1, \ldots, 1}_{m}\},
$$

and let $M(a ; b \mid l ; m)$ denote the set of all nonnegative integral $2 a+b$ by $2 l+m$ matrices $M=\left(M_{i j}\right)$ such that

(i) $C(a, b) M C(l, m)=M$ and

(ii) $M_{i j}$ is even whenever both $i>2 a$ and $j>2 l$.

Suppose $W(\alpha ; \beta)$ and $W(\lambda ; \mu)$ are Weyl subgroups, where the associated partitions have lengths $a, b$ and $l, m$, respectively. Define $M(\alpha ; \beta \mid \lambda ; \mu)$ to be the set of all $M \in M(a ; b \mid l ; m)$ such that the row sums of $M$ are

$$
\alpha_{1}, \alpha_{1}, \ldots, \alpha_{a}, \alpha_{a}, 2 \beta_{1}, \ldots, 2 \beta_{b}
$$

and the column sums of $M$ are

$$
\lambda_{1}, \lambda_{1}, \ldots, \lambda_{l}, \lambda_{l}, 2 \mu_{1}, \ldots, 2 \mu_{m} .
$$

Finally, let $N(\alpha ; \beta \mid \lambda ; \mu)$ be the set of elements of $M(\alpha ; \beta \mid \lambda ; \mu)$ of the form

$$
\left(\begin{array}{c|c}
* & * \\
\hline * & O_{b, m}
\end{array}\right) .
$$


THEOREM 3.1. Let $\langle$,$\rangle denote the usual inner product of characters and suppose$ $W(\alpha ; \beta), W(\lambda ; \mu)$ are Weyl subgroups in $B_{n}$. Then

$$
\langle 1(\alpha ; \beta), 1(\lambda ; \mu)\rangle=|M(\alpha ; \beta \mid \lambda ; \mu)|=\langle\varepsilon(\alpha ; \beta), \varepsilon(\lambda ; \mu)\rangle
$$

and

$$
\langle 1(\alpha ; \beta), \varepsilon(\lambda ; \mu)\rangle=|N(\alpha ; \beta \mid \lambda ; \mu)| \text {. }
$$

Remark. This should be compared to Coleman [6, Theorem 15] and Snapper [52].

Proof. By the Mackey subgroup theorem,

$$
\langle 1(\alpha ; \beta), 1(\lambda ; \mu)\rangle=\sum_{y \in \Delta}\left\langle\left. 1_{W(\alpha ; \beta)}\right|_{Y},\left.1_{W(\lambda ; \mu)}\right|_{Y}\right\rangle
$$

where $Y=W(\alpha ; \beta) \cap(W(\lambda ; \mu))^{y}$ and $\Delta$ is a complete system of $(W(\alpha ; \beta), W(\lambda ; \mu))$ double coset representatives in $B_{n}$. Since each term in this sum is 1 , we have

$$
\langle 1(\alpha ; \beta), 1(\lambda ; \mu)\rangle=|\Delta|=\text { the number of } W(\lambda ; \mu) \text { orbits on } B_{n} / W(\alpha ; \beta) \text {. }
$$

Let $A_{1}, \ldots, A_{a}, B_{1}, \ldots, B_{b}$ and $L_{1}, \ldots, L_{l}, M_{1}, \ldots, M_{m}$ be the admissible partitions of $\Omega$ associated with $W(\alpha ; \beta)$ and $W(\lambda ; \mu)$, respectively. View the coset $g W(\alpha ; \beta)$, $g \in B_{n}$, as the admissible partition $g A_{1}, \ldots, g A_{a}, g B_{1}, \ldots, g B_{b}$ of "type" $(\alpha ; \beta)$. We claim that $g W(\alpha ; \beta)$ and $h W(\alpha ; \beta)$ are in the same $W(\lambda ; \mu)$-orbit if and only if

$$
\begin{aligned}
\left|g\left(A_{i}^{+}\right) \cap L_{j}^{+}\right| & =\left|h\left(A_{i}^{+}\right) \cap L_{j}^{+}\right| \quad \text { for } 1 \leqslant i \leqslant a, 1 \leqslant j \leqslant l, \\
\left|g\left(A_{i}^{-}\right) \cap L_{j}^{+}\right| & =\left|h\left(A_{i}^{-}\right) \cap L_{j}^{+}\right| \quad \text { for } 1 \leqslant i \leqslant a, 1 \leqslant j \leqslant l, \\
\left|g B_{i} \cap L_{j}\right| & =\left|h B_{i} \cap L_{j}\right| \quad \text { for } 1 \leqslant i \leqslant b, 1 \leqslant j \leqslant l, \\
\left|g A_{i} \cap M_{j}\right| & =\left|h A_{i} \cap M_{j}\right| \text { for } 1 \leqslant i \leqslant a, 1 \leqslant j \leqslant m, \\
\left|g B_{i} \cap M_{j}\right| & =\left|g B_{i} \cap M_{j}\right| \text { for } 1 \leqslant i \leqslant b, 1 \leqslant j \leqslant m .
\end{aligned}
$$

It is clear that these conditions are necessary, since for example

$$
\left|x\left(g\left(A_{i}^{+}\right) \cap L_{j}^{+}\right)\right|=\left|x g\left(A_{i}^{+}\right) \cap L_{j}^{+}\right| \quad \text { for } x \in W(\lambda, \mu)
$$

and all relevant $i, j$. To see that (3.2) is also sufficient, observe for example, that the group $A_{L_{1}} \leqslant W(\lambda ; \mu)$ acts as the full symmetric group on $L_{1}^{+}$and acts trivially on $\Omega-L_{1}$. Consequently there is an $x \in A_{L_{1}}$ taking $g\left(A_{1}^{ \pm}\right) \cap L_{1}^{+}$to $h\left(A_{1}^{ \pm}\right) \cap L_{1}^{+}$and $g B_{k} \cap L_{1}^{+}$to $h B_{k} \cap L_{1}^{+}$, since these two partitions of $L_{1}^{+}$have the same type. A similar observation holds for each group $A_{L_{j}}, 2 \leqslant j \leqslant l$, and each group $B_{M_{j}}$, $1 \leqslant j \leqslant m$.

This shows that the orbit parameters given in (3.2) completely determine a $W(\lambda ; \mu)$-orbit on $B_{n} / W(\alpha ; \beta)$. Thus, each element of $\Delta$ is associated with a $2 a+b$ 
by $2 l+m$ matrix of the following form:

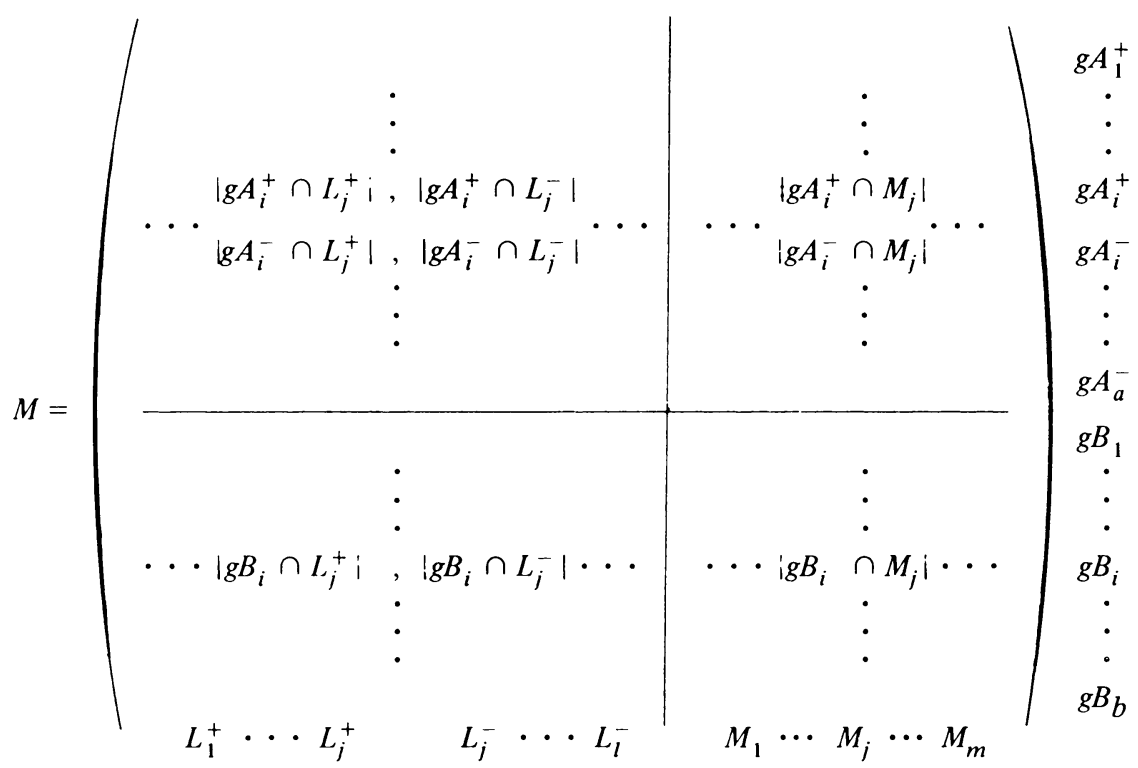

The sum of the entries in one of the rows of this matrix is just the cardinality of the set labeling that row, and similarly for columns. Since $\{\{x,-x\} \mid x=1, \ldots, n\}$ is a system of imprimitivity for the action of $B_{n}$ on $\Omega$, we have

$$
\begin{aligned}
\left|g A_{i}^{+} \cap L_{j}^{+}\right| & =\left|g A_{i}^{-} \cap L_{j}^{-}\right|, & & \left|g A_{i}^{-} \cap L_{j}^{+}\right|=\left|g A_{i}^{+} \cap L_{j}^{-}\right|, \\
\left|g A_{i}^{+} \cap M_{i}\right| & =\left|g A_{i}^{-} \cap M_{i}\right|, & & \left|g B_{i} \cap L_{j}^{+}\right|=\left|g B_{i} \cap L_{j}^{-}\right|,
\end{aligned}
$$

and it follows that $M \in M(\alpha ; \beta \mid \lambda ; \mu)$.

We leave to the reader the verification that any element of $M(\alpha ; \beta \mid \lambda ; \mu)$ may be viewed as a matrix of intersection numbers as in (3.3), and hence determines an element in $\Delta$, from which it follows that

$$
\langle 1(\alpha ; \beta), 1(\lambda ; \mu)\rangle=|M(\alpha ; \beta \mid \lambda ; \mu)| \text {. }
$$

The Mackey subgroup theorem also implies that

$$
\langle\varepsilon(\alpha ; \beta), \varepsilon(\lambda ; \mu)\rangle=\sum_{y \in \Delta}\left\langle\left.\varepsilon\right|_{Y},\left.\varepsilon\right|_{Y}\right\rangle=|\Delta|,
$$

where $Y=W(\alpha ; \beta) \cap W(\lambda ; \mu)^{y}$ and $\Delta$ is a complete system of $(W(\alpha ; \beta), W(\lambda ; \mu))$ double coset representatives as above. Therefore

$$
\langle\varepsilon(\alpha ; \beta), \varepsilon(\lambda ; \mu)\rangle=|M(\alpha ; \beta \mid \lambda ; \mu)| .
$$

In fact, the same argument establishes the remaining equality provided only that the elements of $N(\alpha ; \beta \mid \lambda ; \mu)$ correspond to those double coset representatives $y \in \Delta$ having the property:

$$
\left\langle 1_{Y}, \varepsilon_{Y}\right\rangle=1 \quad \text { where } Y=W(\alpha ; \beta) \cap W(\lambda ; \mu)^{y} .
$$


This condition that $Y \leqslant \operatorname{ker} \varepsilon$ amounts to the condition that none of the various intersections considered in (3.3) contains both $\pm k, 1 \leqslant k \leqslant n$. These are just the elements of $N(\alpha ; \beta \mid \lambda ; \mu)$, and the theorem follows.

COROLlary 3.4. $\langle 1(\alpha ; \beta), 1(\lambda ; \mu)\rangle$ is the coefficient of

$$
\prod_{i=1}^{a}\left(x_{i} x_{-i}\right)^{\alpha_{i}} \prod_{i=1}^{b}\left(y_{i} y_{-i}\right)^{\beta_{i}} \prod_{j=1}^{l}\left(z_{j} z_{-j}\right)^{\lambda} \prod_{j=1}^{m}\left(w_{j} w_{-j}\right)^{\mu_{j}}
$$

in the expansion of the generating function

$$
\Pi \frac{\left(1-x_{i} x_{-i} z_{j} z_{-j}\right)}{\left(1-x_{i} z_{j}\right)\left(1-x_{-i} z_{-j}\right)\left(1-x_{i} x_{-i} w_{j}^{2}\right)\left(1-y_{i}^{2} z_{j} z_{-j}\right)\left(1-y_{i}^{2} w_{i}^{2}\right)}
$$

Theorem 3.1 can be used to express the irreducible characters of $B_{n}$ and the permutation characters $1(\alpha ; \beta)$ as linear combinations of one another, since the characters $1(\alpha ; \beta)$ span the character ring of $B_{n}$ (Mayer [35, 3.1]). We will, however, not require this much information.

The irreducible characters of $B_{n}$ are also labeled by ordered pairs of partitions $(\alpha \mid \beta)$ where $\alpha+s, \beta+n-s$ and $(\alpha \mid \beta)$ occurs as a constituent of $1(\beta ; \alpha)$ (Kerber $[34,2.22])$. A routine, though tedious calculation yields Tables I and II. An example is given after (3.7). We note in passing that rows $1,2,4,5,8,9$ and 10 of Table I can be found on p. 154 of Murnaghan [42].

TABLE I. The irreducible constituents of selected permutation

\begin{tabular}{|c|c|c|c|c|c|c|c|c|c|c|c|c|c|c|}
\hline & $\frac{\sigma}{\Xi}$ & $\begin{array}{l}\frac{\sigma}{=} \\
i \\
1 \\
\Xi\end{array}$ & $\begin{array}{l}\Xi \\
\equiv \\
1 \\
\Xi\end{array}$ & $\begin{array}{l}\frac{\sigma}{2} \\
i \\
i \\
1 \\
\Xi\end{array}$ & $\begin{array}{l}\underline{0} \\
= \\
\approx \\
i \\
1 \\
=\end{array}$ & $\begin{array}{l}\equiv \\
\equiv \\
\tilde{N} \\
1 \\
\Xi\end{array}$ & $\begin{array}{l}\frac{\widehat{v}}{N} \\
1 \\
\Xi\end{array}$ & $\begin{array}{l}\frac{\sigma}{m} \\
m \\
1 \\
\vdots\end{array}$ & $\begin{array}{l}\frac{\sigma}{0} \\
\tilde{i} \\
\dot{m} \\
1 \\
\Xi\end{array}$ & $\begin{array}{l}\frac{\sigma}{0} \\
\tilde{m} \\
\dot{m} \\
1 \\
\Xi\end{array}$ & 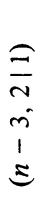 & $\begin{array}{l}\Xi \\
\Xi \\
\bar{m} \\
1 \\
\Xi\end{array}$ & $\begin{array}{l}\approx \\
\equiv \\
\dot{m} \\
1 \\
\Xi\end{array}$ & $\begin{array}{l}\frac{m}{m} \\
1 \\
\Xi\end{array}$ \\
\hline $1(0 ; n)$ & 1 & & & & & & & & & & & & & \\
\hline $1(0 ; n-1,1)$ & 1 & 1 & & & & & & & & & & & & \\
\hline $1(1 ; n-1)$ & 1 & 1 & 1 & & & & & & & & & & & \\
\hline $1(0 ; n-2,2)$ & 1 & 1 & & 1 & & & & & & & & & & \\
\hline $1(0 ; n-2,1,1)$ & 1 & 2 & & 1 & 1 & & & & & & & & & \\
\hline $1(1 ; n-2,1)$ & 1 & 2 & 1 & 1 & 1 & 1 & & & & & & & & \\
\hline $1(2 ; n-2)$ & 1 & 1 & 1 & 1 & & 1 & 1 & & & & & & & \\
\hline $1(0 ; n-3,3)$ & 1 & 1 & & 1 & & & & 1 & & & & & & \\
\hline $1(0 ; n-3,2,1)$ & 1 & 2 & & 2 & 1 & & & 1 & 1 & & & & & \\
\hline $1\left(0 ; n-3,1^{3}\right)$ & 1 & 3 & & 3 & 3 & & & 1 & 2 & 1 & & & & \\
\hline $1(1 ; n-3,2)$ & 1 & 2 & 1 & 2 & 1 & 1 & & 1 & 1 & & 1 & & & \\
\hline $1\left(1 ; n-3,1^{2}\right)$ & 1 & 3 & 1 & 3 & 3 & 2 & & 1 & 2 & 1 & 1 & 1 & & \\
\hline $1(2 ; n-3,1)$ & 1 & 2 & 1 & 2 & 1 & 2 & 1 & 1 & 1 & & 1 & 1 & 1 & \\
\hline $1(3 ; n-3) n \neq 4$ & 1 & 1 & 1 & 1 & & 1 & 1 & 1 & & & 1 & & 1 & 1 \\
\hline $1(3 ; 1)$ & 1 & 1 & 1 & & & 1 & 1 & - & - & & - & & 1 & 1 \\
\hline
\end{tabular}
characters $1(\alpha ; \beta)$ and their multiplicities.

(N.B.-Characters involving nondecreasing sequences are to be ignored.) 
TABLE II. The multiplicities to which the irreducible constituents of $1(1 ; n-1), 1(2 ; n-2)$ and $1(3 ; n-3)$ occur in selected parabolic characters of $B_{n}$ (also the multiplicities to which the irreducible constituents of $\theta_{1}, \theta_{2}$ and $\theta_{3}$ occur in selected parabolic characters of a group with $(B, N)$ )-pair of type $B_{n}$ or $C_{n}$-see $\S 4$ ).

\begin{tabular}{|c|c|c|c|c|c|c|c|c|c|c|}
\hline & $\frac{o}{\Xi}$ & $\begin{array}{l}o \\
\equiv \\
i \\
1 \\
\Xi\end{array}$ & $\begin{array}{l}\text { 三 } \\
\overline{1} \\
\vdots \\
\Xi\end{array}$ & 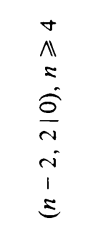 & $\begin{array}{l}m \\
\Lambda \\
= \\
\dot{\Xi} \\
\vdots \\
\dot{\sim} \\
1 \\
\Xi\end{array}$ & $\begin{array}{l}\hat{N} \\
\hat{\Sigma} \\
\hat{I} \\
\frac{\hat{N}}{1} \\
\underline{\Xi}\end{array}$ & $\begin{array}{l}0 \\
\Lambda \\
= \\
\hat{\sigma} \\
\frac{m}{m} \\
\dot{m} \\
1 \\
\Xi\end{array}$ & $\begin{array}{l}n \\
\wedge \\
\equiv \\
\hat{\Xi} \\
\bar{N} \\
m \\
1 \\
\vdots\end{array}$ & 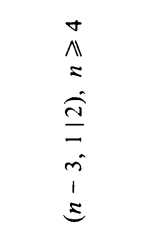 & $\begin{array}{l}\hat{m} \\
\Sigma \\
\dot{m} \\
\frac{m}{m} \\
1 \\
\Xi\end{array}$ \\
\hline Character degree & 1 & $n-1$ & $n$ & $\frac{n(n-3)}{2}$ & $n(n-2)$ & $\left(\begin{array}{l}n \\
2\end{array}\right)$ & $\left(\begin{array}{l}n \\
2\end{array}\right) \frac{n-5}{3}$ & $\left(\begin{array}{c}n \\
2\end{array}\right)(n-4)$ & $\left(\begin{array}{l}n \\
2\end{array}\right)(n-3)$ & $\left(\begin{array}{l}n \\
3\end{array}\right)$ \\
\hline $1(1 ; n-1) n \geqslant 2$ & 1 & 1 & 1 & 0 & 0 & 0 & 0 & 0 & 0 & 0 \\
\hline $1(2 ; n-2) n \geqslant 3$ & 1 & 1 & 1 & 1. & 1 & 1 & 0 & 0 & 0 & 0 \\
\hline $1(3 ; n-3) n \geqslant 5$ & 1 & 1 & 1 & 1 & 1 & 1 & 1 & 1 & 1 & 1 \\
\hline $1(3 ; 1)$ & 1 & 1 & 1 & 0 & 1 & 1 & - & - & 1 & 1 \\
\hline $1(4 ; n-4) n \geqslant 7$ & 1 & 1 & 1 & 1 & 1 & 1 & 1 & 1 & 1 & 1 \\
\hline $1(4 ; 2)$ & 1 & 1 & 1 & 1 & 1 & 1 & 0 & 1 & 1 & 1 \\
\hline $1(4 ; 1)$ & 1 & 1 & 1 & 0 & 1 & 1 & - & 0 & 1 & 1 \\
\hline $1(n ; 0)$ & 1 & 0 & 1 & 0 & 0 & 1 & 0 & 0 & 0 & 1 \\
\hline $1\left(1^{2} ; n-2\right) n \geqslant 3$ & 1 & 2 & 2 & 1 & 2 & 1 & 0 & 0 & 0 & 0 \\
\hline $1(2,1 ; n-3) n \geqslant 5$ & 1 & 2 & 2 & 2 & 3 & 2 & 1 & 2 & 2 & 1 \\
\hline $1(2,1 ; 1)$ & 1 & 2 & 2 & 1 & 3 & 2 & - & - & 2 & 1 \\
\hline $1\left(1^{3} ; n-3\right) n \geqslant 4$ & 1 & 3 & 3 & 3 & 6 & 3 & 1 & 3 & 3 & 1 \\
\hline $1\left(1^{3} ; 0\right)$ & 1 & 2 & 3 & - & 3 & 3 & - & - & - & 1 \\
\hline $1(3,1 ; n-4) n \geqslant 7$ & 1 & 2 & 2 & 2 & 3 & 2 & 2 & 3 & 3 & 2 \\
\hline $1(3,1 ; 2)$ & 1 & 2 & 2 & 2 & 3 & 2 & 1 & 3 & 3 & 2 \\
\hline $1(3,1 ; 1)$ & 1 & 2 & 2 & 1 & 3 & 2 & -- & 1 & 3 & 2 \\
\hline $1(n-1,1 ; 0) n \geqslant 4$ & 1 & 1 & 2 & 0 & 1 & 2 & 0 & 0 & 1 & 2 \\
\hline $1(2,1 ; 0)$ & 1 & 1 & 2 & - & 1 & 2 & - & - & -- & 1 \\
\hline
\end{tabular}

Since this paper was first written, Geissinger and Kinch [20] has appeared. These tables may be calculated directly from the elegant Theorem III.5 of [20].

This completes the data required to deal with groups of type $B_{n}$. The characters of $D_{n}$ are related to those of $B_{n}$ in a particularly simple manner.

Theorem 3.5 (Kerber [34, 2.25]). An irreducible character $(\alpha \mid \beta)$ of $B_{n}$ remains irreducible when restricted to $D_{n}$ if and only if $\alpha \neq \beta$. The characters of the form $(\alpha \mid \alpha)$ decompose into a sum of two irreducible characters $(\alpha \mid \alpha)^{ \pm}$conjugate in $B_{n}$. In any case, $\left.(\alpha \mid \beta)\right|_{D_{n}}=\left.(\beta \mid \alpha)\right|_{D_{n}}$.

Corollary 3.6. Let $W(\lambda ; \mu)$ be a Weyl subgroup of $B_{n}$ and define

$$
W^{*}(\lambda ; \mu)=W(\lambda ; \mu) \cap D_{n}, \quad 1^{*}(\lambda ; \mu)=1_{W^{*}(\lambda ; \mu)}^{D_{n}} \text { for } \mu \neq 0
$$

and

$$
\begin{array}{ll}
W^{*}(\lambda ; 0)^{+}=W(\lambda ; 0) \cap D_{n}, & 1^{*}(\lambda ; 0)^{+}=1_{W^{*}(\lambda ; 0)^{+}}^{D_{n}} \\
W^{*}(\lambda ; 0)^{-}=\left(W(\lambda ; 0)^{\sigma} \cap D_{n}\right), & 1(\lambda ; 0)^{-}=1_{W^{*}(\lambda ; 0)^{-}}^{D_{n}}
\end{array}
$$


where $\sigma$ is the permutation $(1,-1)$ in $S_{\Omega}$. Also, define $(\alpha \mid \beta)$ to be the restriction of the $B_{n}$-character $(\alpha \mid \beta)$ to $D_{n}$. Finally, set $1^{*}(\lambda ; 0)=1^{*}(\lambda ; 0)^{+}+1^{*}(\lambda ; 0)^{-}$. Then

$$
\left\langle 1^{*}(\lambda ; \mu),(\alpha \mid \beta)\right\rangle=\langle 1(\lambda ; \mu)+\varepsilon(\lambda ; \mu),(\alpha \mid \beta)\rangle
$$

and

$$
\left\langle 1^{*}(\alpha ; \beta), 1^{*}(\lambda ; \mu)\right\rangle=|M(\alpha ; \beta \mid \lambda ; \mu)|+|N(\alpha ; \beta \mid \lambda ; \mu)|
$$

ProOF. Since $D_{n}=\operatorname{ker} \varepsilon$,

$$
1^{*}(\lambda ; \mu)^{B_{n}}=1_{W^{*}(\lambda ; \mu)}^{B_{n}}=(1+\varepsilon)_{W(\lambda ; \mu)}^{B_{n}}=1(\lambda ; \mu)+\varepsilon(\lambda ; \mu)
$$

for $\mu \neq 0$. In case $\mu=0$,

$$
1^{*}(\lambda ; 0)^{B_{n}}=\left(1_{W^{*}(\lambda ; 0)^{+}}+1_{W^{*}(\lambda ; 0)^{-}}\right)^{B_{n}}=2\left(1_{W^{*}(\lambda ; 0)^{+}}+1_{W^{*}(\lambda ; 0)^{+}}\right)^{B_{n}},
$$

since $W^{*}(\lambda ; 0)^{+}$and $W^{*}(\lambda ; 0)^{-}$are conjugate in $B_{n}$. However, $W^{*}(\lambda ; 0)^{+}=W(\lambda ; 0)$ and $1_{W(\lambda ; 0)}=\varepsilon_{W(\lambda ; 0)}$, so

$$
1^{*}(\lambda ; 0)^{B_{n}}=\left(1_{W(\lambda ; 0)}+\varepsilon_{W(\lambda ; 0)}\right)^{B_{n}}=1(\lambda ; 0)+\varepsilon(\lambda ; 0) .
$$

The first claim now follows from Frobenius reciprocity.

By the Mackey subgroup theorem, $1^{*}(\lambda ; \mu)=1(\lambda ; \mu)_{D_{n}}$. Consequently, if $1(\lambda ; \mu)=\sum c_{\gamma \delta}(\gamma \mid \delta)$ for integers $c_{\gamma \delta}$, then $\cdot 1^{*}(\lambda ; \mu)=\sum c_{\gamma \delta}(\gamma \mid \delta)$ also. Thus,

$$
\begin{aligned}
& \left\langle 1^{*}(\alpha ; \beta), 1^{*}(\lambda ; \mu)\right\rangle=\left\langle 1^{*}(\alpha ; \beta), \sum c_{\gamma \delta}(\gamma \mid \delta)\right\rangle \\
& \quad=\langle 1(\alpha ; \beta)+\varepsilon(\alpha ; \beta), 1(\lambda ; \mu)\rangle=|M(\alpha ; \beta \mid \lambda ; \mu)|+|N(\alpha ; \beta \mid \lambda ; \mu)|
\end{aligned}
$$

by the first part of this corollary and (3.1).

COROllary 3.7. (i)

$$
\left\langle 1^{*}(\lambda ; \mu),(\alpha \mid \alpha)^{+}\right\rangle=\left\langle 1^{*}(\lambda ; \mu),(\alpha \mid \alpha)^{-}\right\rangle \quad \text { if } \mu \neq 0 .
$$

(ii) If $\alpha \neq \beta$ and if $\left\langle 1^{*}(3 ; n-3) ;(\alpha \mid \beta)\right\rangle \neq 0$, then

$$
\left\langle 1^{*}(\lambda ; \mu),(\alpha \mid \beta)\right\rangle=\langle 1(\lambda ; \mu),(\alpha \mid \beta)\rangle
$$

whenever $\mu+t$ with $t>3$, while

(iii)

$$
\left\langle 1^{*}(\lambda ; 0)^{+},(\alpha \mid \beta)\right\rangle=\left\langle 1^{*}(\lambda ; 0)^{-},(\alpha \mid \beta)\right\rangle=\langle 1(\lambda ; 0),(\alpha \mid \beta)\rangle .
$$

$$
\begin{aligned}
\left\langle 1^{*}(\lambda ; 0)^{+},(\alpha \mid \alpha)^{+}\right\rangle & =\left\langle 1^{*}(\lambda ; 0)^{-},(\alpha \mid \alpha)^{+}\right\rangle=\left\langle 1^{*}(\lambda ; 0)^{+},(\alpha \mid \alpha)^{-}\right\rangle \\
& =\left\langle 1^{*}(\lambda ; 0)^{-},(\alpha \mid \alpha)^{-}\right\rangle .
\end{aligned}
$$

Proof. Statement (i) follows from (3.6) and the fact that $1^{*}(\lambda ; \mu)$ is $B_{n}$-invariant.

If $\mu$ t $t$ and $t>3$, then $N(\lambda ; \mu \mid 3 ; n-3)=0$, and hence (ii) holds in this case.

Clearly, $N(\lambda ; 0 \mid \alpha ; \beta)=M(\lambda ; 0 \mid \alpha ; \beta)$ for all partition pairs $(\alpha ; \beta)$. Thus, $\left\langle 1^{*}(\lambda ; 0),(\alpha \mid \beta)\right\rangle=2\langle 1(\lambda ; 0),(\alpha \mid \beta)\rangle$. Here, $(\alpha \mid \beta)$ is $B_{n}$-invariant while $1^{*}(\lambda ; 0)^{ \pm}$ is not, so that

$$
\left\langle 1^{*}(\lambda ; 0)^{ \pm},(\alpha \mid \beta)\right\rangle=\frac{1}{2}\left\langle 1^{*}(\lambda ; 0),(\alpha \mid \beta)\right\rangle=\langle 1(\lambda ; 0),(\alpha \mid \beta)\rangle .
$$

This completes the proof of (ii). 
Write

$$
1^{*}(\lambda ; 0)^{+}=\sum_{\alpha \neq \beta} c_{(\alpha \mid \beta)}(\alpha \mid \beta)+\sum c_{\alpha}(\alpha \mid \alpha)^{+}+\sum d_{\alpha}(\alpha \mid \alpha)^{-}
$$

for integers $c_{(\alpha \mid \beta)}, c_{\alpha}$ and $d_{\alpha}$. Since $\left(W^{*}(\lambda ; 0)^{+}\right)^{\sigma}=W^{-}(\lambda ; 0)^{-}$, we can conjugate by $\sigma$ (cf. (3.6)) in order to obtain

$$
1^{*}(\lambda ; 0)^{--}=\sum c_{(\alpha \mid \beta)}(\alpha \mid \beta)+\sum c_{\alpha}(\alpha \mid \alpha)^{-}+\sum d_{\alpha}(\alpha \mid \alpha)^{+} .
$$

We claim that $0=\left\langle 1^{*}(\lambda ; 0)^{+}, 1^{*}(\lambda ; 0)^{+}-1^{*}(\lambda ; 0)^{-}\right\rangle$. For, let $\left\{a_{i} \mid 1 \leqslant i \leqslant t\right\}$ be a complete set of $\left(W^{*}(\lambda ; 0)^{+}, W^{*}(\lambda ; 0)^{+}\right)$double coset representatives in $D_{n}$. Then $\left\{a_{i} \sigma \mid 1 \leqslant i \leqslant t\right\}$ is a complete set of $\left(W^{*}(\lambda ; 0)^{+}, W^{*}(\lambda ; 0)^{-}\right)$double coset representatives; for, $w^{+} a \sigma w^{-}=b \sigma$ if and only if $w^{+} a \sigma w^{-} \sigma=b$, while $\sigma w^{-} \sigma \in W^{*}(\lambda ; 0)^{+}$whenever $w^{-} \in W^{*}(\lambda ; 0)^{-}$. This proves our claim.

Thus,

$$
\begin{aligned}
0 & =\left\langle 1^{*}(\lambda ; 0), \sum\left(c_{\alpha}-d_{\alpha}\right)(\alpha \mid \alpha)^{+}+\sum\left(d_{\alpha}-c_{\alpha}\right)(\alpha \mid \alpha)^{-}\right\rangle \\
& =\sum\left(c_{\alpha}-d_{\alpha}\right)\left\langle 1^{*}(\lambda ; 0),(\alpha \mid \alpha)^{+}-(\alpha \mid \alpha)^{-}\right\rangle=\sum\left(c_{\alpha}-d_{\alpha}\right)^{2}
\end{aligned}
$$

This proves (iii).

Further inner products, not handled in (3.7) and Table II, will be needed later. These are found in Table III, which is obtained by further straightforward calculations, as in the following example.

ExAmple. We illustrate the use of (3.1), Table I and (3.6) in a very simple situation. For $n \geqslant 3$ we show that

$$
\begin{aligned}
1(n-2,1 ; 1) & =(n \mid 0)+2(n-1,1 \mid 0)+2(n-1 \mid 1)+\chi, \\
1^{*}(n-2,1 ; 1) & =(n \mid 0)+2(n-1,1 \mid 0)+3(n-1 \mid 1)+\chi^{\prime}
\end{aligned}
$$

where $\langle\chi, 1(1 ; n-1)\rangle=0=\left\langle\chi^{\prime}, 1^{*}(1 ; n-1)\right\rangle$. We see trivially that the elements of $M(0 ; n \mid n-2,1 ; 1)$ and $M(0 ; n-1,1 \mid n-2,1 ; 1)$ are $(n-2, n-2,1,1,2)$ and

$$
\begin{gathered}
\left(\begin{array}{ccccc}
n-2 & n-2 & 1 & 1 & 0 \\
0 & 0 & 0 & 0 & 2
\end{array}\right), \quad\left(\begin{array}{cccccc}
n-2 & n-2 & 0 & 0 & 2 \\
0 & 0 & 1 & 1 & 0
\end{array}\right), \\
\left(\begin{array}{ccccc}
n-3 & n-3 & 1 & 1 & 2 \\
1 & 1 & 0 & 0 & 0
\end{array}\right),
\end{gathered}
$$

respectively. It is almost as easy to see that $M(1 ; n-1 \mid n-2,1 ; 1)$ consists of

$$
\begin{aligned}
& \left(\begin{array}{ccccc}
1 & 0 & 0 & 0 & 0 \\
0 & 1 & 0 & 0 & 0 \\
n-3 & n-3 & 1 & 1 & 2
\end{array}\right), \quad\left(\begin{array}{ccccc}
0 & 1 & 0 & 0 & 0 \\
1 & 0 & 0 & 0 & 0 \\
n-3 & n-3 & 1 & 1 & 2
\end{array}\right), \\
& \left(\begin{array}{ccccc}
0 & 0 & 1 & 0 & 0 \\
0 & 0 & 0 & 1 & 0 \\
n-2 & n-2 & 0 & 0 & 1
\end{array}\right), \quad\left(\begin{array}{ccccc}
0 & 0 & 0 & 1 & 0 \\
0 & 0 & 1 & 0 & 0 \\
n-2 & n-2 & 0 & 0 & 2
\end{array}\right)
\end{aligned}
$$

and

$$
\left(\begin{array}{ccccc}
0 & 0 & 0 & 0 & 1 \\
0 & 0 & 0 & 0 & 1 \\
n-2 & n-2 & 1 & 1 & 0
\end{array}\right)
$$


TABLE III. The multiplicities of the irreducible constituents of $1^{*}(3, n-3)$ in selected parabolic characters of $D_{n}$ (also the multiplicities of the irreducible constituents of $\theta_{1}, \theta_{2}$ and $\theta_{3}$ in selected parabolic characters of a group with a $(B, N)$-pair of type $D_{n}$-see $\S 4$ ).

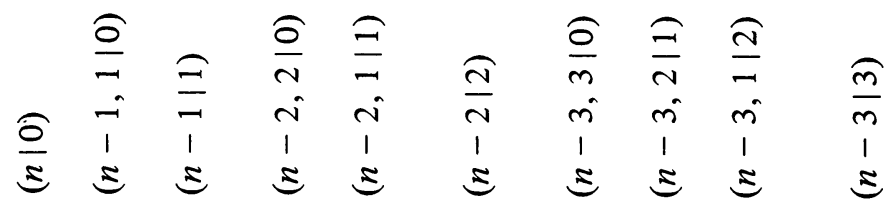

\begin{tabular}{|c|c|c|c|c|c|c|c|c|c|c|}
\hline $1^{*}(1 ; 3)$ & 1 & 1 & 1 & 0 & 0 & $0+0$ & - & - & 0 & $m$ \\
\hline $1 *(2 ; 2)$ & 1 & 1 & 1 & 1 & 1 & $1+1$ & - & - & 0 & $\stackrel{m}{\Xi}$ \\
\hline $1^{*}(3 ; 1)$ & 1 & 1 & 2 & 0 & 1 & $1+1$ & - & - & 1 & $\xi$ \\
\hline $1^{*}\left(1^{2} ; 2\right)$ & 1 & 2 & 2 & 1 & 2 & $1+1$ & - & - & 0 & ن \\
\hline $1^{*}(2,1 ; 1)$ & 1 & 2 & 3 & 1 & 4 & $2+2$ & - & - & 3 & ळ్ \\
\hline $1^{*}\left(1^{3} ; 1\right)$ & 1 & 3 & 4 & 2 & 8 & $3+3$ & - & - & 6 & \\
\hline $1^{*}(2 ; 3)$ & 1 & 1 & 1 & 1 & 1 & 1 & - & 0 & 0 & \\
\hline $1^{*}\left(1^{2} ; 3\right)$ & 1 & 2 & 2 & 1 & 2 & 1 & - & 0 & 0 & a \\
\hline $1^{*}(3 ; 2)$ & 1 & 1 & 1 & 1 & 1 & 2 & - & 1 & 1 & $\underline{\Xi}$ \\
\hline $1^{*}(2,1 ; 2)$ & 1 & 2 & 2 & 2 & 3 & 3 & - & 2 & 3 & $\overline{0}$ \\
\hline $1^{*}\left(1^{3} ; 2\right)$ & 1 & 3 & 3 & 3 & 6 & 4 & - & 3 & 5 & $\stackrel{\Perp}{\mathscr{N}}$ \\
\hline $1^{*}(3,1 ; 1)$ & 1 & 2 & 3 & 1 & 4 & 4 & - & 1 & 4 & \\
\hline $1^{*}(3 ; 3)$ & 1 & 1 & 1 & 1 & 1 & 1 & 1 & 1 & 1 & $1+1$ \\
\hline $1^{*}(2,1 ; 3)$ & 1 & 2 & 2 & 2 & 3 & 2 & 1 & 2 & 2 & $1+1$ \\
\hline $1^{*}(3,1 ; 2)$ & 1 & 2 & 2 & 2 & 3 & 3 & 1 & 3 & 1 & $2+2$ \\
\hline $1^{*}(3,1 ; 3)$ & 1 & 2 & 2 & 2 & 3 & 2 & 2 & 3 & 3 & 3 \\
\hline $1 *(4 ; 3)$ & 1 & 1 & 1 & 1 & 1 & 1 & 1 & 1 & 1 & 2 \\
\hline $1^{*}(4,1 ; 2)$ & 1 & 2 & 2 & 2 & 4 & 2 & 1 & 3 & 2 & 2 \\
\hline
\end{tabular}

By (3.1) we have

$$
\begin{aligned}
\langle 1(0 ; n), 1(n-2,1 ; 1)\rangle & =1, \\
\langle 1(0 ; n-1,1), 1(n-2,1 ; 1)\rangle & =3
\end{aligned}
$$

and

$$
\langle 1(1 ; n-1), 1(n-2,1 ; 1)\rangle=5 .
$$

The first three rows of Table I imply $\langle(0 \mid n), 1(n-2,1 ; 1)\rangle=1,\langle(n-1,1 \mid 0)$, $1(n-2,1 ; 1)\rangle=3-1=2$ and $\langle(n-1 \mid 1), 1(n-2,1 ; 1)\rangle=5-3=2$. The first 
claim follows. By (3.6) and the above enumeration,

$$
\begin{aligned}
\left\langle 1^{*}(0 ; n), 1^{*}(n-2,1 ; 1)\right\rangle & =1+0=1, \\
\left\langle 1^{*}(0 ; n-1,1), 1^{*}(n-2,1 ; 1)\right\rangle & =3+0=3
\end{aligned}
$$

and

$$
\left\langle 1^{*}(1 ; n-1), 1^{*}(n-2,1 ; 1)\right\rangle=5+1=6,
$$

since the last matrix listed in $M(1, n-1 \mid n-2,1 ; 1)$ above is in $N(1, n-1 \mid$ $n-2,1,1)$. The second claim follows from (3.7) (each of the characters $(n \mid 0)$, $(n-1,1 \mid 0),(n-1 \mid 1)$ is an irreducible character of $\left.D_{n}\right)$ and from Table I, just as above.

The last Weyl group we must discuss is the symmetric group $S_{n}$. Since $S_{n}$ is a homomorphic image of $B_{n}$, some of the irreducible characters of $B_{n}$ are also characters of $S_{n}$. It turns out that these characters are precisely those of the form $(\alpha \mid \beta)$ where $\beta=0$ (this follows, for example, from Mayer [35, 1.1]). In fact, one can obtain a table describing characters of the symmetric group from Table II by deleting columns $3,5,6,8,9$, and 10 , and replacing the semicolons in the row labels by commas and rearranging into partitions.

The information we require about characters of $S_{n}$ is included in the following lemma.

LEMMA 3.8. Let $\theta_{i}$ be the permutation character of $S_{n}$ on $i$-sets (alias $\left.1(n-i, i)\right), \theta_{i j}$ $(i<j)$ the character on pairs consisting of an $i$-set and a $j$-set containing it (alias $1(n-j, j-i, i))$, and so on. Then the following hold for $1 \leqslant i \leqslant n / 2$.

(i) $\theta_{i}-\theta_{i-1}$ is irreducible, $\theta_{0}=1$.

(ii) $\left\langle\theta_{i}-\theta_{i-1}, \theta_{i-1}\right\rangle=0$.

(iii) $\theta_{i j}=\theta_{j-i, j}$.

(iv) $\left\langle\theta_{1}-1, \theta_{12 \ldots n-1}\right\rangle n-1$.

(v) $\left\langle\theta_{3}-\theta_{2}, \theta_{34}\right\rangle=\left\langle\theta_{4}-\theta_{3}, \theta_{45}\right\rangle=2$ if $n>8$.

(vi) $\left\langle\theta_{2}-\theta_{1}, \theta_{12}\right\rangle=1 ;\left\langle\theta_{2}-\theta_{1}, \theta_{13}\right\rangle$ is 1 if $n=4$, and is 2 if $n>4$; $\left\langle\theta_{2}-\theta_{1}, \theta_{123}\right\rangle=3$; and if $n>5$ then $\left\langle\theta_{2}-\theta_{1}, \theta_{124}\right\rangle=4$ and $\left\langle\theta_{2}-\theta_{1}, \theta_{1234}\right\rangle=6$.

For these inner products, see Murnaghan [42, p. 154], where more standard notation is used; or see (10.1) below.

4. Parabolic characters. In this section we are concerned with characters of $\operatorname{Aut}(\operatorname{Chev}(V))$. After a technical lemma that is used to deal with graph automorphisms in $\S \S 8-10$, we turn to characters of parabolic type. We recall the fundamental correspondence, due to Curtis, Iwahori and Kilmoyer [8, (7.2)], between these characters and those of the Weyl group, which provides the link with §3. Finally, in (4.7) we use the remarkable formulae of Hoefsmit [26] to completely determine the characters of parabolic type that fail to have degree divisible by $p$ (compare Howlett [27]). 
Lemma 4.1. Let $G$ be as in (1.1) or (1.2), and let $G^{+}=G \cap G^{\natural}$, where $G^{\natural}$ is generated by $\operatorname{Chev}(V)$ and its diagonal and field automorphisms. Let $K \leqslant G$ satisfy $G=K G^{+}$, and set $K^{+}=K \cap G^{+}$. Write $\varphi=1_{K}^{G}=1+\chi+\zeta$ where $\chi$ is irreducible, and let $\varphi^{+}=\left.\varphi\right|_{G^{+}}, \chi^{+}=\left.\chi\right|_{G^{+}}$and $\zeta^{+}=\left.\zeta\right|_{G^{+}}$. Finally, let $B$ be a Borel subgroup of $G^{+}(c f .[9,(2.6)])$. Then

(a) $\varphi^{+}=\left(1_{K^{+}}\right)^{G^{+}}$;

(b) Each irreducible constituent of $\chi^{+}$occurs to multiplicity one;

(c) The irreducible constituents of $\chi^{+}$are conjugate under $G$;

(d) Either $\chi^{+} \subseteq 1_{B}^{G^{+}}$or $\left\langle\chi^{+}, 1_{B}^{G^{+}}\right\rangle=0$.

Remarks. (1) $\Gamma(V)=G^{\natural}$ unless $V$ has type $\Omega^{+}(2 n, q)$ in which case $\left|\Gamma(V): G^{\natural}\right|=2$.

(2) A slightly more technical, similar lemma that can be used to restrict to $\operatorname{Chev}(V)$ is described in $\S 11$.

Proof. Since $K^{+}=K \cap G^{+}, g \in G^{+}$fixes a coset $K h, h \in G^{+}$, if and only if it fixes $K^{+} h$. This proves (a). Since $\left|G: G^{+}\right|$divides 6, Isaacs [28, (6.18)] (applied twice if necessary) implies (b), and (c) follows from Clifford's theorem [28, (6.5)]. Finally, (d) follows from (b) and (c) together with the fact that $1_{B}^{G^{+}}$is $G$-invariant $[9$, (2.6)].

The $(B, N)$ structure of $\operatorname{Chev}(V)$ is discussed in detail in Carter [3] (and also Curtis, Kantor and Seitz [9, §2]). We observe that each of the groups $G$ in Theorem 1.1 or 1.2 has a $(B, N)$-pair with the associated Coxeter system $(W, R)$, $R=\left\{s_{1}, \ldots, s_{n}\right\}$ of type $B_{n}, C_{n}, D_{n}$ or $A_{n}$.

For each subset $I \subseteq\{1, \ldots, n\}, W_{I}=\left\langle s_{j} \mid j \in I\right\rangle$ and $G_{I}=B W_{I} B$ are parabolic subgroups. (This is consistent with $\S 3$, where $s_{i}=(i, i+1)(-i,-i-1)$ for $i<n$ while $s_{n}=(-n, n)$, and $W=W\left(B_{n}\right) \cong W\left(C_{n}\right)$.) The irreducible characters $\zeta$ of $G$ that occur as constituents in $1_{G_{I}}^{G}$ are said to be of parabolic type.

It is elementary to see that the normalizer of a Sylow $p$-subgroup of $G$ fixes a unique flag in $V$. The group $B$ is just such a normalizer $[3, \S 8.6],[9, \S 2]$, and so fixes a flag $\left(V_{1}, V_{2}, \ldots\right)$. The group $G_{V_{i}}$ is maximal in $G$ and contains $B$. Consequently, $G_{V_{i}}$ is the parabolic subgroup $G_{i}=G_{\{i\}}$ for a suitable labeling of the elements of $R$ (cf. $[3,8.3 .3])$. It follows that the parabolic subgroup $G_{I}$ is the stabilizer of an $\left(i_{1}, i_{2}, \ldots\right)$-flag, where $I=\left\{i_{1}, i_{2}, \ldots\right\}$; and the character $\theta_{I}=\theta_{i_{1} i_{2}} \cdots=1_{G}^{B}$ is just the permutation character of $G$ on the set of all $\left(i_{1}, i_{2}, \ldots\right)$-flags.

Theorem 7.2 of Curtis, Iwahori and Kilmoyer [8] provides a natural 1-1 correspondence $\zeta \rightarrow \zeta_{0}$ between the irreducible characters $\zeta$ of parabolic type and the characters $\zeta_{0}$ of $W$ such that

$$
\left\langle\zeta, \theta_{I}\right\rangle=\left\langle\zeta, 1_{G_{I}}^{G}\right\rangle=\left\langle\zeta_{0}, 1_{W_{I}}^{W}\right\rangle=\left\langle\zeta_{0}, 1(\lambda ; \mu)\right\rangle,
$$

where $I=\left\{i_{1}, i_{2}, \ldots, i_{t}\right\}, \quad \lambda$ is obtained by rearranging the sequence $\left(i_{1}, i_{2}-i_{1}, \ldots, i_{t}-i_{t-1}\right)$ into a partition of $i_{t}$, and $\mu=\left(n-i_{t}\right)$, provided that $i_{t}<n$ when $G$ has type $D_{n}$. (The flags of groups of type $D_{n}$ have the more complex structure described at the beginning of $\S 2$, but $\lambda$ is obtained in an analogous way.) The examples most relevant to (1.1) are $\theta_{i}=1(i ; n-i), \theta_{1 i}=1(i-1, i ; n-i)$, and $\theta_{123}=1\left(1^{3} ; n-3\right)(\mathrm{cf}$. Table II). 
A consequence of the fact that the right-hand side of (4.2) depends only on the partition $(\lambda ; \mu)$ and not on $I$ is that

$$
\theta_{r, s}=\theta_{s-r, s}
$$

for appropriate values of $r, s$.

For simplicity of notation we will use $(\alpha \mid \beta)$ to denote the irreducible character of $W$ as well as the corresponding parabolic character of $G$.

The importance of (4.2) cannot be over-emphasized. The proof of Theorem 1.1 essentially reduces to the situation where one of the characters in $1_{K}^{G}$ corresponds under (4.2) to a character labeling a column of Table II. In each case, the table forces $K$ to have so much transitivity of one sort or another that it cannot escape recognition.

The following is a simple example of (4.2).

COROllary 4.4. Let $\operatorname{Chev}(V) \leqslant G^{+} \leqslant G \leqslant \Gamma(V)$, and assume that $G$ and $G^{+}$have the same Weyl group. Let $B$ be a Borel subgroup of $G$, and let $B^{+}=B \cap G^{+}$. If $\chi$ is an irreducible constituent of $1_{B}^{G}$, then $\left.\chi\right|_{G^{+}}$is an irreducible constituent of $1_{B^{+}}^{G^{+}}$.

Proof. Apply (4.2) to both $G$ and $G^{+}$.

A few entries of Tables II and III can be computed using the following special case of Curtis, Iwahori and Kilmoyer [8, (2.2) and (9.17)].

THEOREM 4.5. (i) The multiplicity to which the character $\zeta$ in (4.2) occurs in $1_{B}^{G}$ is the degree of the associated character $\zeta_{0}$.

(ii) If $\rho$ is the character of $G$ corresponding to the reflection character $(n-1 \mid 1)$ of $W\left(B_{n}\right)$ or $W\left(D_{n}\right)$, then

$$
n-|I|=\left\langle\rho, \theta_{I}\right\rangle=\left\langle(n-1 \mid 1), 1_{W_{l}}^{W}\right\rangle .
$$

As an example of the use of (4.5 ii), we will give another proof of the fact that $\left\langle\theta_{1 n-1},(n-1,1 \mid 0)\right\rangle=2$ for groups of type $D_{n}$ (cf. \$3). First, compute $\left\langle\theta_{1 n-1}, \theta_{1}\right\rangle=6$ by considering the action of the stabilizer of a $(1, n-1)$-flag on points. Then note that $\theta_{1 n-1}=\theta_{1 n n}$ is the character of triples $\left(V_{1}, V_{n}, V_{n}^{\prime}\right)$ with $V_{n} \cap V_{n}^{\prime}$ an $n-1$-space on $V_{1}$. By $\left(4.5\right.$ ii), $\left\langle\theta_{1 n-1},(n-1 \mid 1)\right\rangle=3$. Since $\theta_{1}=1+(n-1 \mid 1)+(n-1,1 \mid 0)$, the desired result follows.

In his thesis [26], Hoefsmit gives formulae for the generic degrees for all irreducible representations of the generic ring corresponding to a classical group. These formulae require numerous preliminary definitions.

The index parameters $x$ and $y$ of $G$ are defined by

$$
x=\left|B: B \cap B^{s_{1}}\right|, \quad y=\left|B: B \cap B^{s_{n}}\right| .
$$

The index parameters of a classical group associated with the field $G F(q)$ are powers of $q$ and are given explicitly in Curtis, Kantor and Seitz [9, §5].

The crux of our discussion concerns groups of type $B_{n}$, since groups of type $D_{n}$ correspond to the situation $y=1$ and groups of type $A_{n-1}$ correspond to the situation $y=0$ (Hoefsmit [26, (3.4.12), (3.4.15)]). It is important to note that $x>1$ in every case. 
Let $\alpha$ be a partition of $s$ and let $\alpha$ also denote the associated Young diagram (cf. Kerber [34]). The conjugate partition $\bar{\alpha}$ is associated with the diagram obtained from $\alpha$ by interchanging the rows and columns of $\alpha$. The $(i, j)$-hook of $\alpha$ for a node $(i, j)$ of $\alpha$ is $A_{i j}^{\alpha} \cup\{(i, j)\} \cup L_{i j}^{\alpha}$ where $A_{i j}^{\alpha}=$ nodes $(i, t)$ in $\left.\alpha \mid t>j\right\}$ is the hook's arm and $L_{i j}^{\alpha}=\{$ nodes $(t, j)$ in $\alpha \mid t>j\}$ is the hook's leg. The $(i, j)$ hook length of $\alpha$ is

$$
h_{i j}^{\alpha}=\left|A_{i j}^{\alpha} \cup L_{i j}^{\alpha} \cup\{(i, j)\}\right|=\left(\alpha_{i}-j\right)+\left(\bar{\alpha}_{j}-i\right)+1 .
$$

Hook lengths were first introduced by Nakayama [45] in connection with modular representations of the symmetric groups. They play an important role in the intricate recursion relations that arise in the study of the symmetric groups.

Let $(\alpha ; \beta)$ be a partition pair as in $\S 3$, where $\alpha \vdash s, \beta \vdash(n-s)$. The $(i, j)$-split hook lengths are defined by $g_{i j}^{\alpha}=\left(\alpha_{i}-j\right)+\left(\bar{\beta}_{j}-i\right)+1$ for each node $(i, j)$ of $\alpha$ and $g_{i j}^{\beta}=\left(\beta_{i}-j\right)+\left(\bar{\alpha}_{j}-i\right)+1$ for each node $(i, j)$ in $\beta$. (Caution: A split hook length may be negative; for example, if $(\alpha ; \beta)=\left(1^{2} ; 0\right)$ then $g_{2,1}^{\alpha}=-1$.) Following Hoefsmit [26], we define the following rational functions of $x$ and $y$ :

$$
\begin{aligned}
H_{i j}^{\alpha} & =x^{i-\bar{\alpha}_{j}}\left(x^{h_{i j}^{\alpha}}-1\right) /(x-1), \\
G_{i j}^{\alpha} & =1+y x^{g_{i j}^{\alpha}}, \\
H_{i j}^{\beta} & =x^{i-\bar{\beta}_{j}}\left(x^{h_{i j}^{\beta}}-1\right) /(x-1), \\
G_{i j}^{\beta} & =x^{-\bar{\alpha}_{j}}\left(y^{-1} x^{g_{i j}^{\beta}}+1\right),
\end{aligned}
$$

and the Poincaré polynomial

$$
P(x, y)=\prod_{i=0}^{n-1}\left\{\left(1+x^{i} y\right)\left(x^{i+1}-1\right) /(x-1)\right\} .
$$

Hoefsmit gives the following formula for the degree $d(\alpha \mid \beta)$ of the irreducible character $(\alpha \mid \beta)$ of $G$ associated with the Weyl group character $(\alpha \mid \beta)$ by (4.2).

$$
d(\alpha \mid \beta)=P(x, y) /\left(\prod_{(i, j) \in \alpha} H_{i j}^{\alpha} G_{i j}^{\alpha} \prod_{(i, j) \in \beta} H_{i j}^{\beta} G_{i j}^{\beta}\right) .
$$

THEOREM 4.7. Suppose $(\alpha \mid \beta)$ is not $1=(n \mid 0)$ and $G$ has type $A_{n}, B_{n}, C_{n}$, or $D_{n}$, $n \geqslant 2$. Then $p$ divides $d(\alpha \mid \beta)$ unless $G$ of type $\operatorname{Sp}(2 n, 2)$ and $(\alpha \mid \beta)=(n-1,1 \mid 0)$, $(n-1 \mid 1)$ or $(0 \mid n)$. In these cases

$$
\begin{gathered}
d(n-1,1 \mid 0)=\left(1+2^{n}\right)\left(2^{n-1}-1\right), \\
d(n-1 \mid 1)=\left(1+2^{n-1}\right)\left(2^{n}-1\right)
\end{gathered}
$$

and

$$
d(0 \mid n)=\left(1+2^{n}\right)\left(1+2^{n-1}\right) / 3 .
$$

Remarks. (1). The first two exceptional cases of (4.7) are the nontrivial irreducible constituents of the 2-transitive permutation representations of $\operatorname{Sp}(2 n, 2)$. While they do not yield exceptional cases for (1.1), they do appear in the characters of the imprimitive rank 3 representations of $\operatorname{Sp}(2 n, 2)=\Omega(2 n+1,2)$ on the cosets of $\Omega^{ \pm}(2 n, 2)$. 
(2). The case of $G$ of type $A_{n}$ in (4.7) is treated in Curtis, Kantor and Seitz [9, (5.9)] by a more elementary argument.

(3). In (4.7), the case of untwisted $G$ of type $B_{n}, C_{n}$ with $q>2$, or $D_{n}$ is due to Howlett [27].

Proof. Consider the product

$$
\prod_{(i, j) \in \boldsymbol{\alpha}} H_{i j}^{\alpha}=\prod_{(i, j) \in \boldsymbol{\alpha}} x^{i-\bar{\alpha}_{,}}\left(\frac{x^{h_{1}^{\alpha}}-1}{x-1}\right)=\prod_{(i, j) \in \boldsymbol{\alpha}}\left(\frac{x^{h_{1 j}^{\alpha}}-1}{x-1}\right) / x^{\Sigma\left(\bar{\alpha}_{j}-i\right)} .
$$

Here,

$$
\sum_{(i, j) \in \alpha}\left(\bar{\alpha}_{j}-i\right)=\sum_{j}\left[\sum_{i=1}^{\bar{\alpha}_{j}}\left(\bar{\alpha}_{j}-i\right)\right]=\sum_{j}\left[\begin{array}{c}
\bar{\alpha}_{j} \\
\sum_{i=1} i
\end{array}\right]=\sum_{j}\left(\begin{array}{c}
\bar{\alpha}_{j} \\
2
\end{array}\right),
$$

and each factor of the form $\left(x^{h}-1\right) /(x-1)$ is congruent to 1 modulo $p$. It follows from (4.6) that

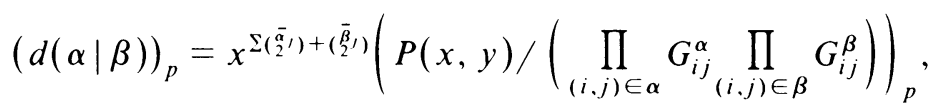

where $(Q)_{p}$ denotes the highest power of $p$ dividing $Q$. Next, observe that

$$
\prod_{(i, j) \in \alpha} G_{i j}^{\alpha} \prod_{(i, j) \in \beta} G_{i j}^{\beta}=\prod_{(i, j) \in \alpha}\left(1+y x^{\left.g_{i j}^{\alpha}\right)} \prod_{(i, j) \in \beta}\left(1+y^{-1} x^{g_{i j}^{\beta}}\right) / x^{\Sigma},\right.
$$

where

$$
\sum=\sum_{(i, j) \in \beta} \bar{\alpha}_{j}=\sum_{j}\left(\sum_{i=1}^{\bar{\beta}_{j}} \bar{\alpha}_{j}\right)=\sum_{j} \bar{\alpha}_{j} \bar{\beta}_{j}
$$

Since

$$
\left(\begin{array}{c}
\bar{\alpha}_{j} \\
2
\end{array}\right)+\left(\begin{array}{c}
\bar{\beta}_{j} \\
2
\end{array}\right)+\bar{\alpha}_{j} \bar{\beta}_{j}=\frac{1}{2}\left(\bar{\alpha}_{j}+\bar{\beta}_{j}\right)\left(\bar{\alpha}_{j}+\bar{\beta}_{j}-1\right)=\left(\begin{array}{c}
\bar{\alpha}_{j}+\bar{\beta}_{j} \\
2
\end{array}\right),
$$

we now have

$$
(d(\alpha \mid \beta))_{p}=x^{\Sigma\left(\bar{\alpha}_{2} \bar{\beta}^{-},\right)}\left\{\frac{P(x, y)}{\prod_{(i j) \in \alpha}\left(1+y x^{\left.g_{\prime \prime}^{\alpha}\right)} \prod_{(i, j) \in \beta}\left(1+y^{-1} x^{\left.g_{\prime \prime}^{\beta}\right)}\right.\right.}\right\}_{p} .
$$

(i) Suppose $p \neq 2$. Then the only way a factor in the denominator can influence $(d(\alpha \mid \beta))_{p}$ is if it has the form

$$
1+p^{-k}=p^{-k}\left(1+p^{k}\right) .
$$

In this case it contributes a positive power of $p$. Therefore,

$$
(d(\alpha \mid \beta))_{p} \geqslant x^{\Sigma\left(\bar{\alpha}_{2}+\bar{\beta}_{1}\right)} .
$$

Since $x>1$ is a power of $p,(d(\alpha \mid \beta))_{p}=1$ only if $\bar{\alpha}_{j}+\bar{\beta}_{j} \leqslant 1$ for all $j$, that is, $(\alpha \mid \beta)=(n \mid 0)$ or $(0 \mid n)$. But if $(\alpha \mid \beta)=(0 \mid n)$, then $g_{i j}^{\beta}=0$ and $G_{11}^{\beta}=1+y^{-1}$. 
Therefore,

$$
(d(\alpha \mid \beta))_{p}=\frac{y}{(1+y)_{p}}\left(\frac{P(x, y)}{\prod_{i=2}^{n}\left(1+y^{-1} x^{i-1}\right)}\right)_{p} \geqslant y .
$$

This shows that $d(0 \mid n)$ is divisible by $p$ also, unless perhaps $y=1$. If $y=1, G$ is of type $D_{n}$ and $(0 \mid n)=(n \mid 0)$, by (3.5) and (4.3).

(ii) Suppose $p=2$. Then there is the possibility that a factor in the denominator has the form $1+1=2$. Observe that $g_{i j}^{\alpha}$ and $g_{i j}^{\beta}$ are both strictly decreasing functions of $i$ (as $\alpha_{i} \geqslant \alpha_{i+1}, \beta_{i} \geqslant \beta_{i+1}$ by the definition of a partition of integers). Therefore, factors of this troublesome type occur at most $\bar{\alpha}_{1}+\bar{\beta}_{1}$ times in (4.8). Set $x=2^{m}$ and $\gamma_{j}=\bar{\alpha}_{j}+\bar{\beta}_{j}$. Then (4.8) yields

$$
\begin{aligned}
\log _{2}\left[(d(\alpha \mid \beta))_{2}\right] & \geqslant m\left(\sum\left(\begin{array}{c}
\bar{\alpha}_{j}+\bar{\beta}_{j} \\
2
\end{array}\right)\right)-\bar{\alpha}_{1}-\bar{\beta}_{1} \\
& =\frac{m}{2}\left[\gamma_{1}\left(\gamma_{1}-\left(1+\frac{2}{m}\right)\right)\right]+m \sum_{j=2}^{n}\left(\begin{array}{c}
\gamma_{j} \\
2
\end{array}\right) .
\end{aligned}
$$

Now $\gamma_{j}$ is an integer so each summand is nonnegative, and the first term is nonpositive only on the interval $0 \leqslant \gamma_{1} \leqslant 1+2 / m \leqslant 3$. It follows easily that $d(\alpha \mid \beta)$ is divisible by $p$, except perhaps if

(a) $\gamma_{1}=3, \gamma_{2} \leqslant 1$ and $m=1$,

(b) $\gamma_{1}=2, \gamma_{2}=2, \gamma_{3} \leqslant 1$ and $m=1$, or

(c) $\gamma_{1}=2, \gamma_{2} \leqslant 1$ and $m \leqslant 2$.

(d) $\gamma_{1}=1$.

Since $\left\{\gamma_{i}\right\}$ is a nonincreasing sequence of natural numbers (it is the sum of two such sequences $)$, the corresponding pairs of partitions are:

(a) $\{\alpha, \beta\}=\{(n-2,1,1) ; \varnothing\},\{(n-2,1) ;(1)\},\{(n-2) ;(1,1)\}$,

(b) $\{\alpha, \beta\}=\{(n-2,2) ; \varnothing\},\{(n-2) ;(2)\}$,

(c) $\{\alpha, \beta\}=\{(n-1) ;(1)\}$.

(d) $\{\alpha, \beta\}=\{(n) ; \varnothing\}$.

Direct computation of (4.8) shows that 2 divides $d(\alpha \mid \beta)$ in each case except (c) and (d) when $m=1$ and $x=y=2$. These index parameters are those of $\operatorname{Sp}(2 n, 2)$ (Curtis, Kantor and Seitz [9, Tables 2 and 5]). This completes the proof of (4.7) in case $G$ has type $B_{n}$ or $C_{n}$.

If $G$ has type $A_{n}$, then (4.6) still holds with $y=0$ and $\beta$ เ 0 (Hoefsmit [26, 3.4.12]), and so the above calculation also establishes (4.7) in this case.

Suppose $G$ has type $D_{n}$. Then $y=1$ and $(\alpha \mid \beta)$ is irreducible only if $\alpha \neq \beta$ (Hoefsmit [26, 3.4.15]). Since $G \geqslant \Omega^{+}(2 n, q), G$ is of index 2 in a subgroup $G^{*}$ of $\Gamma^{+}(2 n, q)$ of type $B_{n}$. By Clifford's theorem, the irreducible characters of $G$ that do not extend to characters of $G^{*}$ are conjugate in $G^{*}$, and hence have degree a half of the expression (4.6). Therefore, the above argument proves (4.7) for $G$, except perhaps for characters of the form $(\alpha \mid \alpha)$ when $y=1, p=2$ and the right-hand side 
of (4.8) is not divisible by 4 . In this case, split hook lengths are hook lengths and hence are positive, and

$$
4 \nmid\left(x^{\sum\left(\frac{2 \bar{\alpha}_{j}}{2}\right)} \frac{P(x, 1)}{\left[\prod_{(i, j) \in \alpha}\left(1+x^{g_{i j}^{\alpha}}\right)\right]^{2}}\right)=\frac{2 x^{\sum\left(\frac{2 \bar{\alpha}_{j}}{2}\right)}}{\left[\prod_{(i, j) \in \alpha}\left(1+x^{\left.h_{i j}^{\alpha}\right)}\right]_{2}^{2}\right.} \geqslant 2 x^{n},
$$

contrary to the hypothesis that $n \geqslant 2$. This completes the proof of (4.7).

5. Transitivity in small dimensions. In this long section, we will consider classical groups of low rank: those of rank 2 or 3 , as well as $\mathrm{\Gamma O}^{+}(8, q)$. The goals are a number of results concerning subgroups having specific transitivity properties. Most of these are closely related to (1.1) or (11.1). However, some are designed for use in $\S 6$, where arbitrary dimensional situations are reduced, in part, to small dimensional ones.

There are two approaches to the required transitivity results. One is geometric, ad hoc, tedious, and very long, but reasonably elementary. The other uses classification theorems [14], [43], [44], [61], [64]-[66] which depend upon deep results concerning the classification of finite simple groups. We have chosen the latter approach: it requires much less space, and at the same time yields somewhat stronger results than are essential for our main theorems.

This section is divided into four subsections: (A) invokes the aforementioned classification theorems in order to deal with subgroups of $\Gamma L(4, q)$ and $\Gamma L(5, q)$; (B) uses (A) in order to discuss subgroups of rank 2 classical groups; (C) deals with subgroups of rank 3 classical groups; and (D) concerns $\mathrm{\Gamma O}^{+}(8, q)$.

Recall that $H^{(\infty)}$ denotes the last term of the derived series of $H$. Throughout this section $K^{(\infty)}$ (or $H^{(\infty)}$ ) will be uniquely determined up to conjugacy in $\Gamma(V)$, unless otherwise stated. Since $K^{(\infty)}$ is usually irreducible on $V, K / Z(K) K^{(\infty)}$ is a group of outer automorphisms of $K^{(\infty)}$, and hence can be determined at a glance.

(A) Subgroups of $\Gamma L(4, q)$ and $\Gamma L(5, q)$. Throughout this subsection, let $H \leqslant \Gamma L(W)=\Gamma L(4, q)$ or $\Gamma L(5, q)$. The possibilities for $H$ have been more or less determined by Mwene [43], [44], Wagner [61], DiMartino and Wagner [14], Zalesskiĭ [64], [65] and Zalesskiǐ and Suprunenko [66].

THEOREM 5.1. Let $H$ be a primitive subgroup of $\Gamma L(4, q)$. Then one of the following holds.

(a) $H \geqslant S L(4, q)$.

(b) $H \leqslant \Gamma L\left(4, q^{\prime}\right)$ with $G F\left(q^{\prime}\right) \subset G F(q)$.

(c) $H \leqslant \Gamma L\left(2, q^{2}\right)$, with the latter group embedded naturally.

(d) $H \leqslant Z(H) H_{1}$, where $H_{1}$ is an extension of a special group of order $2^{6}$ by $S_{5}$ or $S_{6}$. Here, $q$ is odd, $H_{1}$ induces a monomial subgroup of $O^{+}(6, q)$, and $H_{1}$ is uniquely determined up to $\Gamma L(4, q)$-conjugacy.

(e) $H^{(\infty)}$ is $S p(4, q)^{\prime}$ or $S U\left(4, q^{1 / 2}\right)$.

(f) $H \leqslant \Gamma O^{*}(4, q)$.

(g) $H^{(\infty)}$ is $\operatorname{PSL}(2, q)$ or $\operatorname{SL}(2, q)$ (many classes). 
(h) $H^{(\infty)}$ is $A_{5}$. Here, $H$ arises from the natural permutation representation of $S_{5}$ in $O(5, q)$, and $p \neq 2,5$.

(i) $H^{(\infty)}$ is $2 \cdot A_{5}, 2 \cdot A_{6}$ or $2 \cdot A_{7}$. These arise from the natural permutation representation of $S_{7}$ in $O(7, q)$.

(j) $H^{(\infty)}=A_{7}$, and $p=2$.

(k) $H^{(\infty)}=S p(4,3)$, and $q \equiv 1(\bmod 3)$. This arises from the natural representation of the Weyl group $W\left(E_{6}\right)$ in $O^{+}(6, q)$.

(1) $H^{(\infty)}=S L(2,7)$, and $q^{3} \equiv 1(\bmod 7)$. Here, $H^{(\infty)}$ lies in the group $2 \cdot A_{7}$ occurring in (i).

(m) $H^{(\infty)}=4 \cdot \operatorname{PSL}(3,4)$, and $q$ is a power of 9 .

(n) $H^{(\infty)}=S z(q)$, and $p=2$.

PRoof. If $q$ is even, see [43], [65]. (It is necessary to sift through the various cases examined in these references in the course of proving their main theorems.)

If $q$ is odd, we will use [44]. (Note that [66] assumes that $p>5$.)

By primitivity, if $H$ has a noncentral abelian normal subgroup then (c) holds. Suppose that (c) does not hold, but that $H$ has a noncentral normal subgroup $E$ of prime power order. Since $\operatorname{dim} V=4, E$ is a 2-group. We may assume that $E / Z(E)$ is elementary abelian, and then (d) follows readily.

Let $\bar{M}=M / Z(H)$ be a minimal normal subgroup of $\bar{H}=H / Z(H)$. We may assume that $\bar{M}$ is a direct product $\bar{S}_{1} \times \cdots \times \bar{S}_{k}$ of isomorphic nonabelian simple groups $\overline{S_{i}}$, where $\overline{S_{i}}=S_{i} / Z(H)$.

If $S_{1}$ is reducible, then (by Clifford's theorem) $M$ preserves a decomposition $W=T_{1} \oplus T_{2}$, where $T_{1}$ and $T_{2}$ are $S_{1}$-isomorphic 2-spaces. Then $S_{1}$ fixes a regulus $\Sigma$ of $q+1$ lines, which are permuted by $N_{H}\left(S_{1}\right)$. Thus, $N_{H}\left(S_{1}\right) \leqslant \Gamma L(4, q)_{\Sigma}$ $=\Gamma O^{+}(4, q)$. If $k=1$, then (f) holds. If $k>1$, then $S_{1} \times S_{2} \leqslant \Omega^{+}(4, q) Z(H)$, from which (f) again follows.

Finally, suppose that $S_{1}$ is irreducible. Then $M=S_{1}$, and $K^{(\infty)}$ is quasisimple. This is precisely the situation studied by Mwene [44]. His theorem, and his study of the modular representations of covering groups of $S_{1}$, complete the proof.

For further information concerning $(5.1 \mathrm{~m})$, see Finkelstein [16], McLaughlin [38] and Mwene [44].

THEOREM 5.2. Let $H$ be a primitive subgroup of $\Gamma L(5, q)$. Then one of the following holds.

(a) $H \geqslant S L(5, q)$.

(b) $H \leqslant \Gamma L\left(5, q^{\prime}\right)$ with $G F\left(q^{\prime}\right) \subset G F(q)$.

(c) $H \leqslant \Gamma L\left(1, q^{5}\right)$.

(d) $H \leqslant Z(H) H_{1}$, where $H_{1}$ is an extension of an extraspecial group of order $5^{3}$ by $S L(2,5)$. Here, $H_{1}$ is uniquely determined up to $\Gamma L(5, q)$-conjugacy, and $p \neq 5$.

(e) $H^{(\infty)}=\Omega(5, q)$ or $S U\left(5, q^{1 / 2}\right)$.

(f) $H^{(\infty)}$ is $\operatorname{PSL}(2, q)$, in the representation afforded by homogeneous polynomials of degree 4 in 2 variables.

(g) $H^{(\infty)}=\Omega(5,3)$, and $q \equiv 1(\bmod 6)$. 
(h) $H^{(\infty)}$ is $A_{5}, A_{6}$ or $A_{7}$, and is contained in $\Omega(5, q)$. Here, $p=7$ if $H^{(\infty)}=A_{7}$. These cases arise from the permutation module for PGL(2,5), $S_{6}$ or $S_{7}$.

(i) $H^{(\infty)}=\operatorname{PSL}(2,11), p \neq 11$. This arises from the irreducible complex representation of $P S L(2,11)$ of degree $\frac{1}{2}(11-1)$.

(j) $H^{(\infty)}=M_{11}, p=3$ (two classes).

Proof. See [61], [65] for $q$ even and [14] for $q$ odd. (Note that [66] assumes that $p>5$.)

REMARK. The precise fields over which the groups listed in $(5.1,2)$ can be realized are discussed at length in [14], [44], [61].

Various transitivity and related results are simple consequences of $(5.1,2)$.

Corollary 5.3. Let $H$ be a subgroup of $\Gamma L(4, q)$ of order divisible by a primitive divisor $r$ of $q^{2}+1$. Then one of the following holds.

(i) $H \geqslant S L(4, q)$.

(ii) $H \leqslant \Gamma L\left(2, q^{2}\right)$.

(iii) $H^{(\infty)}=S p(4, \mathrm{q})^{\prime}$.

(iv) $H \leqslant \Gamma O^{-}(4, q)$.

(v) $H=A_{7}<S L(4,2)$.

(vi) $H^{(\infty)}=S z(q)$.

(vii) $r=5$, and one of (5.1 d, h, i, j, k, 1, or m) holds.

Proof. Clearly, $H$ is primitive, so we only need to sift through the list in (5.1). Note that $r \geqslant 5$ and $r \neq 7$. If $r=5$ then (vii) can occur. All remaining cases of (5.1) are easily checked.

REMARK 5.4. We will use primitive divisors together with $(5.1,2)$ in other situations. It will always be as straightforward to check the lists as it was in (5.3). Another example is provided by the next result.

COROllary 5.5. If $H$ is a subgroup of $\Gamma L(n, q), n=4$ or 5 , and if $H$ is transitive on points, then one of the following holds.

(a) $H \geqslant S L(n, q)$.

(b) $H \leqslant \Gamma L\left(1, q^{n}\right)$.

(c) $H \leqslant \Gamma L\left(2, q^{2}\right)<\Gamma L(4, q)$.

(d) $H^{(\infty)}=S p(4, q)^{\prime}<\Gamma L(4, q)$.

(e) $H=A_{7}$, inside $S L(4,2)$.

(f) $H$ has a normal extraspecial subgroup of order $2^{5}$, and 5||$H \mid$, where $H<G L(4,3)$.

Proof. If $n=5$, then $|H|$ is divisible by a primitive divisor $r$ of $q^{5}-1$. Here, $r>11$, and it is straightforward to use (5.2).

If $n=4$, then (5.3) applies. The possibilities listed in (5.3 vi) are easily checked.

(B) Subgroups of rank 2 classical groups. The results in (A) can clearly be used to study $\Gamma \operatorname{Sp}(4, q), \Gamma U(4, q), \Gamma U(5, q), \Gamma O(5, q)$ and $\Gamma O^{-}(6, q)$. We only need to consider the first three of these. $\Gamma S p(4, q)$ was handled long ago by Mitchell [41], when $q$ is odd. (Partial results for $q$ even were obtained by Flesner [18]; he essentially only dealt with the case of subgroups containing transvections.) 
THEOREM 5.6. Let $K \leqslant \Gamma \operatorname{Sp}(4, q)$. Then one of the following holds.

(i) $K^{(\infty)}=S p\left(4, q^{\prime}\right)^{\prime}$ with $G F\left(q^{\prime}\right) \subseteq G F(q)$.

(ii) $K$ fixes a point, a line, a pair of skew lines, or a pair of perpendicular hyperbolic lines.

(iii) $K \cap S p(4, q) \leqslant S p\left(2, q^{2}\right) \cdot 2$.

(iv) $K^{(\infty)}$ is $S L\left(2, q^{\prime}\right)$, in its representation afforded by homogeneous polynomials of degree 3 in 2 variables, where $G F\left(q^{\prime}\right) \subseteq G F(q)$ and $p>3$.

(v) $K \leqslant \Gamma O^{ \pm}(4, q)$, where $p=2$.

(vi) $K^{(\infty)}=S z(q)$, where $q>p=2$.

(vii) $K$ induces a subgroup of $\Gamma O(5, q)$ fixing an anisotropic 2-space.

(viii) $K$ fixes a quintuple of pairwise orthogonal nonsingular points when viewed inside $\Gamma O(5, q)$, and $q$ is odd.

(ix) $K^{(\infty)}$ is $2 \cdot A_{5}$ or $2 \cdot A_{6}$, in the representation obtained from the mod permutation representation of $A_{6}$ in $\Omega^{-}(6, q)$.

(x) $K^{(\infty)}$ is $2 \cdot A_{7}$, in the representation obtained from the mod 7 permutation representation of $S_{7}$ in $O(7, q)$.

Historical Remark. The first $q>2$ for which this was proved was $q=3$. Dickson [11] listed all 70 factors of 25920 , and then sifted through them, primarily using Sylow's theorem and lists of permutation groups of small degree.

THEOREM 5.7. Let $K \leqslant \Gamma U(4, q)$. Then one of the following holds.

(i) $K^{(\infty)}=S U\left(4, q^{\prime}\right)$ with $G F\left(q^{\prime}\right) \subseteq G F(q)$.

(ii) $K$ fixes a point or line, a nonsingular point or line, a pair of skew lines, a pair of perpendicular nonsingular lines, or a quadruple of pairwise perpendicular nonsingular points.

(iii) $\mathrm{K}$ induces a subgroup of $\mathrm{P \Gamma O}^{-}(6, q)$ fixing a nonsingular point or line, a pair of perpendicular nonsingular planes, or (when q is odd) a sextuple of pairwise perpendicular nonsingular points.

(iv) $K^{(\infty)}$ is $2 \cdot A_{7}$ or $S L(2,7)$. Here, $K^{(\infty)}$ arises from the permutation representation of $S_{7}$ in $O(7, q)$.

(v) $K^{(\infty)}=S p(4,3)$, and $q^{3} \equiv 1(\bmod 7)$. Here, $K^{(\infty)}$ arises from the natural representation of $W\left(E_{6}\right)$.

(vi) $K^{(\infty)}=4 \cdot \operatorname{PSL}(3,4)$, and $q$ is a power of 3 .

THEOREM 5.8. Let $K \leqslant \Gamma U(5, q)$. Then one of the following holds.

(i) $K^{(\infty)}=S U\left(5, q^{\prime}\right)$ with $G F\left(q^{\prime}\right) \subseteq G F(q)$.

(ii) $K$ fixes a point or line, a nonsingular point or line, or a quintuple of pairwise perpendicular nonsingular points.

(iii) $K \leqslant Z(K) \Gamma O(5, q)$, where the $O(5, q)$ is embedded naturally.

(iv) $K \leqslant \Gamma U\left(1, q^{5}\right)$.

(v) $K \leqslant Z(K) K_{1}$, where $K_{1}$ is an extension of an extraspcial group of order $5^{3}$ by $S L(2,5)$, and $p \neq 5$.

(vi) $H^{(\infty)}=\Omega(5,3)$, and $p>3$.

(vii) $H^{(\infty)}$ is $A_{5}, A_{6}$, or $A_{7}$, and is contained in $\Omega(5, q)$. Moreover, $p=7$ if $H^{(\infty)}=A_{7}$

(viii) $H^{(\infty)}=\operatorname{PSL}(2,11)$, where $p \neq 11$. 
Theorems 5.6-5.8 are almost immediate consequences of the following elementary result.

Lemma 5.9. Let $H$ be an absolutely irreducible subgroup of $G L(W)=G L(n, q)$. Let $(,)_{1}$ and $(,)_{2}$ be two nonsingular hermitian forms on $W$ (or two nonsingular alternating forms on $W$ ) preserved by $H$. Then there is a scalar $c$ such that $(,)_{1}=$ $c(,)_{2}$.

Proof. View $H$ as a group of matrices, and $(,)_{i}$ as arising from a matrix $A_{i}$. Consider the unitary case. Here, $A_{\mathrm{i}}^{-1} h A_{i}=\left(\bar{h}^{-1}\right)^{t}$ for all $h \in H$. Then $A_{2} A_{1}^{-1}$ centralizes $H$, and hence is a scalar by Schur's Lemma.

Proof of (5.6)-(5.8). We may assume that $K$ is primitive. Suppose first that $K_{0}=K \cap S L(V)$ is absolutely irreducible. By (5.9), if a group occurring in (5.1) or (5.2) can arise in (5.6), (5.7) or (5.8), then all occurrences are conjugate. The groups listed in the latter theorems all occur, for suitable $q[\mathbf{4 4}],[\mathbf{1 4}],[\mathbf{6 0}]$. Note that neither (5.1j) nor (5.2j) can occur: in each case, there is a 1-dimensional subspace $x$ such that $K_{x}^{(\infty)}$ does not fix any hyperplane.

If $K_{0}$ is not absolutely irreducible, then one of $(5.1 \mathrm{c}, \mathrm{f})$ or $(5.2 \mathrm{c})$ holds. Suppose that $K$ lies in both $\Gamma \operatorname{Sp}(4, q)$ and $\Gamma L\left(1, q^{4}\right)$. Then $K_{0}$ has an irreducible normal cyclic $r$-subgroup for some prime $r$ (cf. (2.18)) By Sylow's theorem, $K \leqslant \Gamma \operatorname{Sp}\left(2, q^{2}\right)$, with the latter group embedded naturally. The same argument applies if $K$ is in $\Gamma U(5, q) \cap \Gamma L\left(1, q^{10}\right)$, this time producing $(5.8$ iv). However, the case $\Gamma U(4, q) \cap \Gamma L\left(1, q^{8}\right)$ cannot occur (acting irreducibly), since $|S U(4, q)|$ is not divisible by the required prime $r$.

This leaves us with the possibility that $\operatorname{dim} V=4$ and $(5.1 \mathrm{f}$ ) holds (with $q$ replaced by $q^{2}$ in the unitary case). Then $K$ has a normal subgroup $S$ acting irreducibly on some 2-dimensional subspace $T$. By primitivity, $T$ has at least 3 images under $K$, all of which must be $S$-isomorphic.

If $T$ is a line, then (5.6 vii) or (5.7 iii) holds. (For, $S$ fixes at least 3 points of the corresponding 5- or 6-dimensional orthogonal space.)

If $T$ is nonsingular, then $T^{\perp}$ is also $S$-invariant. Moreover, $T$ and $T^{\perp}$ are $S$-isomorphic. This uniquely determines $S$ up to conjugacy. Then $S$ fixes a nonsingular plane of the corresponding $O(5, q)$ or $O^{-}(6, q)$ space. Consequently, (5.6 vii) or (5.7 iii) holds for $K$. This completes the proof of (5.6)-(5.8).

The remainder of this subsection parallels $(5 \mathrm{~A})$ : primitive divisors will be used in order to deduce transitivity properties.

Lemma 5.10. Let $K \leqslant \Gamma U(4, q)$, and assume that $|K|$ is divisible by a primitive divisor $r$ of $q^{2}+1$. Then one of the following holds.

(i) $K$ has a normal Sylow $r$-subgroup, and fixes a hyperbolic line when viewed inside $\Gamma O^{-}(6, q)$.

(ii) $K$ fixes a line.

(iii) $K^{(\infty)}=S U(4, q)$.

(iv) $K^{(\infty)}=S p(4, q)^{\prime}$.

(v) $K^{(\infty)}=S p\left(2, q^{2}\right)$.

(vi) $K^{(\infty)}=\Omega^{-}(4, q)$. 
(vii) $K^{(\infty)}=S z(q)$.

(viii) $K^{(\infty)}=4 \cdot P S L(3,4)$, and $q$ is a power of 3 .

(ix) $r=5$, and either $K^{(\infty)}$ is $A_{5}$ (with $q$ odd ), $2 \cdot A_{5}, 2 \cdot A_{6}, 2 \cdot A_{7}$ or $S p(4,3)$, or $K \cap S U(4, q)$ induces a monomial group inside $O^{-}(6, q)$.

Proof. Check the lists in (5.6) and (5.7) (using Dickson [12, Chapter 12] in order to deal with subgroups in the cases (5.6 iii, v)).

Lemma 5.11. If $K \leqslant \Gamma U(5, q)$, and if $|K / K \cap Z(\Gamma U(5, q))|$ is divisible by both $q^{2}+1$ and $q^{3}+1$, then either

(i) $K \geqslant S U(5, q)$,

(ii) $K \unrhd Z_{3}^{4} \rtimes A_{5}$, acting monomially inside $\Gamma U(5,2)$,

(iii) $K^{(\infty)}=S U(4, q)$,

(iv) $K^{(\infty)}$ is $A_{5}, A_{6}$ or $E \rtimes A_{5}$, with $E$ extraspecial of order 32 , where $K<\Gamma U(5,2)$ and $K$ fixes a nonsingular point, or

(v) $K^{(\infty)}=4 \cdot P S L(3,4)$, inside $\Gamma U(5,3)$.

Proof. Assume that (i) does not hold. If $q^{3}+1$ has a primitive divisor, then $K$ fixes a nonsingular point by (5.8), and hence (5.10) applies. If no primitive divisor exists, then $q=2$ by (2.18). Thus, $|K|$ is divisible by 45 . If (ii) does not hold, then $K$ fixes a nonsingular point by $(5.8)$, and hence $K \leqslant\left(Z_{3} \times G U(4,2)\right) \cdot 2$. The subgroups of $\Gamma U(4,2)$ of order divisible by 15 give rise to the examples in (iv).

Corollary 5.12. Let $K \leqslant \Gamma \operatorname{Sp}(4, q)$, $\Gamma U(4, q)$ or $\Gamma U(5, q)$. If $K$ is transitive on points or lines, then one of the following holds.

(i) $K^{(\infty)}=\operatorname{Chev}(V)^{\prime}$.

(ii) $K^{(\infty)}=\operatorname{Sp}\left(2, q^{2}\right)$, inside $\Gamma S p(4, q)$.

(iii) $K^{(\infty)}=S L(2,5)<S p\left(2,3^{2}\right)$, inside $\Gamma S p(4,3)$.

(iv) $K^{(\infty)}=\Omega^{-}(4, q)$, inside $\Gamma \operatorname{Sp}(4, q)$ with $q$ even.

(v) $K^{(\infty)}=S U(3, q)$, inside $\Gamma U(4, q)$.

(vi) $K \unrhd S U(3,2)^{\prime \prime}$, inside $\Gamma U(4,2)$ (and $K$ fixes a nonsingular point).

(vii) $K$ has a normal monomial subgroup $Z_{3}^{3} \rtimes A_{4}$, and $K<\Gamma U(4,2)$.

(viii) $K^{(\infty)}=\operatorname{PSL}(3,2)$, inside $\Gamma U(4,3)$ (and $K$ fixes a nonsingular point).

(ix) $K^{(\infty)}=3 \cdot A_{7}$, inside $\Gamma U(4,5)$ (and $K$ fixes a nonsingular point).

(x) $K \unrhd E \rtimes Z_{5}$, where $E$ is extraspecial of order $2^{5}, E \leqslant O_{2}(K), K<\Gamma S p(4,3)$, and $K$ acts monomially within $\Gamma O(5,3)$.

(xi) $K^{(\infty)}=4 \cdot \operatorname{PSL}(3,4)$, inside $\Gamma U(4,3)$.

Proof. If $K \leqslant \Gamma \operatorname{Sp}(4, q)$, then $(q+1)\left(q^{2}+1\right)|| K \mid$. Thus, (5.10) applies by (2.18). All possibilities are easily checked (although some computation is required in the monomial case of $(5.10 \mathrm{ix})$ ).

Let $K \leqslant \Gamma U(4, q)$. If $K$ is point-transitive, then $\left(q^{2}+1\right)\left(q^{3}+1\right)|| K \mid$, and (5.10) again applies. If $K$ is line-transitive, then $(q+1)\left(q^{3}+1\right)|| K \mid$. As in the proof of (5.11), if $q>2$ then a primitive divisor of $q^{3}+1$ can be used: if $K$ is irreducible then (i) or (xi) holds. For any $q,(2.8)$ can be applied in case $K$ fixes a nonsingular point. Finally, if $q=2$ then $P S U(4,2) \cong P S p(4,3)$ and $(q+1)\left(q^{3}+1\right)=27$, so that (5.6) and our transitivity lead to (vi) or (vii). 
If $K \leqslant \Gamma U(5, q)$, then $q^{5}+1|| K \mid$. By (2.18) and (5.8), only (i) can occur.

Definition. $K$ is an $(a, b, c)$-group if it has $a$ point-orbits, $b$ line-orbits and $c$ flag-orbits. The next two results contain complete determinations of all $(a, b, c)$-groups for some triples $(a, b, c)$ of special importance later.

Corollary 5.13. Let $K \leqslant \Gamma \operatorname{Sp}(4, q), \Gamma U(4, q)$ or $\Gamma U(5, q)$, and let $(a, b, c)$ be one of the triples $(1,2,2),(1,2,3),(2,1,2)$ or $(2,1,3)$. If $K$ is an $(a, b, c)$-group, then one of the following holds.

$(1,2,2)_{\mathrm{i}} K^{(\infty)}=\operatorname{Sp}\left(2, q^{2}\right)$, inside $\Gamma \operatorname{SP}(4, q)$.

$(1,2,2)_{\mathrm{ii}} K / Z(K)=Z_{2}^{4} \rtimes D$, where $D$ is dihedral of order $10, K<\Gamma \operatorname{Sp}(4,3)$, and $K$ acts monomially within $\Gamma O(5,3)$ with respect to an orthonormal basis.

$(1,2,2)_{\mathrm{iii}} K /\langle-1\rangle=S_{5}, A_{5} \times Z_{2}$ or $S_{5} \times Z_{2}, K<\Gamma S p(4,3)$, and $K$ acts monomially within $\Gamma O(5,3)$ with respect to an orthonormal basis.

$(1,2,2)_{\mathrm{iv}} K^{(\infty)}=4 \cdot \operatorname{PSL}(3,4)$, inside $\Gamma U(4,3)$.

$(1,2,3)$ There are no $(1,2,3)$-groups.

$(2,1,2)_{\mathrm{i}} K^{(\infty)}=\Omega^{-}(4, q)$, with q even, inside $\Gamma \operatorname{Sp}(4, q)$.

$(2,1,2)_{\mathrm{ii}} K^{(\infty)}=S U(3, q)$, inside $\Gamma U(4, q)$.

$(2,1,2)_{\mathrm{iii}} K<\Gamma U(4,2)$, and either $K \unrhd Z_{3}^{3} \rtimes A_{4}$ with the latter group monomial with respect to an orthonormal basis, or $K$ fixes a nonsingular point and $K \unrhd S U(3,2)^{\prime \prime} \rtimes Z_{8}$.

$(2,1,3)$ There are no $(2,1,3)$-groups.

PROOF. This is a consequence of (5.12) and some straightforward computations.

Lemma 5.14. Let $K \leqslant \Gamma \operatorname{Sp}(4, q), \Gamma U(4, q)$ or $\Gamma U(5, q)$. If $K$ is a $(2,2,3)$-group, then one of the following holds.

(i) $K^{(\infty)}=\operatorname{Sp}\left(4, q^{\prime}\right)$, inside $\Gamma U(4, q)$.

(ii) $K^{(\infty)}=S L(2, q) \times S L(2, q), K$ fixes a pair of perpendicular hyperbolic lines, and $K<\Gamma \operatorname{Sp}(4, q)$.

(iii) $K \unrhd \Omega^{+}(4, q)$, inside $\Gamma \operatorname{Sp}(4, q)$ with $q$ even.

(iv) $K<S p(4,2), K$ behaves as in (ii), and 18||$K \mid$.

(v) $K<\Gamma S p(4,3)$, $K$ behaves as in (ii), and $K \unrhd S L(2,3)^{\prime} \times S L(2,3)^{\prime}$.

(vi) $K / Z(K) \unrhd Z_{2}^{4} \rtimes A_{4}$, where $K<\Gamma \operatorname{Sp}(4,3)$ and $K$ acts monomially inside $\Gamma O(5,3)$ with respect to an orthonormal basis.

(vii) $K / Z(K) \unrhd Z_{2}^{4} \rtimes P S L(2,5)$ or $Z_{2}^{4} \rtimes A_{6}$, where $K<\Gamma U(4,3)$ and $K$ acts monomially inside $\Gamma O^{-}(6,3)$ with respect to an orthonormal basis.

(viii) $K^{(\infty)}=Z_{3}^{4} \rtimes A_{5}$, where the latter group acts monomially inside $\Gamma U(5,2)$ with respect to an orthonormal basis.

(ix) $K^{(\infty)}=S U(4, q)$, inside $\Gamma U(5, q)$.

Proof. There is a point $x$ and a line $L$ such that both $K_{x}^{x^{\perp} / x}$ and $K_{L}^{L}$ are transitive on points.

Suppose first that $K \leqslant \Gamma U(5, q)$, but that (viii) does not hold. Then (5.11) applies, so that $K$ fixes a nonsingular point $b$. Clearly, $K$ must be transitive on the points of $b^{\perp}$, as well as on the $q^{3}\left(q^{4}-1\right)$ points not in $b^{\perp}$. Thus, only (5.11 ii or iii) can occur. 
Next, suppose that $K \leqslant \Gamma U(4, q)$. This time, $|K|$ is divisible by both $q+1$ and $q^{2}+1$, and (5.10) applies. (5.10 i-vii) are easily handled. Consider (5.10 ix). The first four cases lie in $\Gamma \operatorname{SP}(4, q)$, but are not transitive on points there (cf. (5.12)). If $K^{(\infty)}=S p(4,3)$ then $q \geqslant 7$, and hence $q^{2}+1 \nmid|K|$.

Finally, suppose that $K \leqslant \Gamma \operatorname{Sp}(4, q)$. Then $q+1|| K \mid$. Consider the possibilities listed in (5.6). We may assume that $K$ is primitive. This takes care of (5.6 ii), and (5.6 iii) is easily eliminated. In ( $5.6 \mathrm{iv,} \mathrm{vii} \mathrm{and} \mathrm{ix)} \mathrm{there} \mathrm{are} \mathrm{more} \mathrm{than} 2$ point-orbits, while in (5.6 vi) there are 4 flag-orbits.

If $K \unrhd \Omega^{+}(4, q)$, and if $K$ has an element interchanging the two normal subgroups $S L(2, q)$ of $\Omega^{+}(4, q)$, then $K$ has the desired orbit structure. Similarly, this and (v) are the only possibilities arising from $(5.6 \mathrm{v})$.

Finally, in (5.6 viii) it is again straightforward to check the orbit structure.

(C) Rank $n=3$. We now turn to spaces $V$ of rank $n=3$. The only interesting result is (5.19), which characterizes both the groups $G_{2}(q)$ and (for $q=2$ or 8 ) the groups $\Gamma L\left(2, q^{3}\right)$.

The case of $V$ of type $\Omega^{+}(6, q)$ is especially easy, and will usually be ignored in view of the following result.

LEMMA 5.15. A point-transitive subgroup of $\mathrm{\Gamma O}^{+}(6, q)$ either contains $\Omega^{+}(6, q)$ or is $A_{7}$ or $S_{7}$ inside $O^{+}(6,2)$.

Proof. (5.1) (but compare (10.2)).

REMARK. The next two results concern groups $K$ which are transitive on points and planes. The numbers of points, lines and planes are given in $\S 6$, Table IV. By (2.12), if $V$ is not unitary then $K$ is $(1,3)$-transitive.

LEMma 5.16. If $n=3$, then $\Gamma(V)$ has no subgroup transitive on points and planes and having 2 orbits on lines and (1,3)-flags, and 3 orbits on (1,2)-flags.

Proof. Let $K$ be such a subgroup. As just noted, $V$ must be unitary. By hypothesis, there is a line $L$ such that $K_{L}^{L}$ is transitive. An element of $K_{L}$ of order a primitive divisor of $q^{2}+1$ (cf. (2.18)) will fix each plane $E \supset L$. On the other hand, $q^{2}+q+1|| K_{E}^{E} \mid$ by (2.12). By Mitchell [40] and Hartley [22], $K_{E}^{E} \geqslant S L\left(3, q^{2}\right)$, whereas $K$ is not $(1,3)$-transitive.

THEOREM 5.17. If $n=3$, and if $K$ is a subgroup of $\Gamma(V)$ which is transitive on points, lines and planes, then $K \geqslant \operatorname{Chev}(V)$ (or $K$ is $A_{7}$ or $S_{7}$ inside $\left.O^{+}(6,2)\right)$.

Proof. By (2.6), we may assume that $K$ is not flag-transitive. Also, we may assume that $K=O^{p^{\prime}}(K)$. Let $E$ be a plane and $x$ a point of $E$. Set $K^{*}=K_{x}^{x^{1} / x}$. We will consider the various possibilities for $V$ separately.

Case $K<\Gamma S p(6, q)$ or $\Gamma O(7, q)$. Since $K^{*}$ is line-transitive but not flag-transitive, (5.12) can be applied: $K<\Gamma O(7, q)$, and either $K^{*}$ fixes a nonsingular 4-space $F$ or $q=3$ and $K^{*} \unrhd Z_{2}^{4} \rtimes Z_{5}$.

Also, $K_{E}^{E}$ is point-transitive but not flag-transitive. By (2.7), if $\left|K_{x E}^{E}{ }^{x}\right|$ is divisible by $q$ then $q=3$ and $q^{2}$ cannot divide this order. Thus, if $F$ exists then $K^{* F}$ $\Omega^{-}(4, q)$. 
Consequently, $q=3$, and either $K^{*(\infty)}=A_{5}$ or $K^{*} \unrhd Z_{2}^{4} \rtimes Z_{5}$. If $K^{*} \unrhd Z_{2}^{4} \rtimes Z_{5}$, there is an involution $t \in C_{K}(x)$ such that $[V, t]$ has type $\Omega^{+}(4,3)$; then $t$ fixes a plane and acts nontrivially on it, and this contradicts the known behavior of $K_{E}^{E}$. Thus, $K^{*(\infty)}=A_{5}$. Let $A<K_{x}$, where $\left|A^{x^{\perp} / x}\right|=4, A<K^{(\infty)}$, and $A$ is a 2-group. Then 3||$N_{K}(A)_{x} \mid$, and $A$ centralizes $x$. Let $T \in S y l_{3} N_{K}(A)_{x}$. Then $A T$ centralizes nonsingular 2-spaces of both $V$ and $x^{\perp} / x$. Consequently, $T$ centralizes $y^{\perp} / y$ for some point $y$. There is a plane $E^{\prime}$ on $y$ not centralized by $T$. Then $T^{E^{\prime}}$ contains a transvection, and we again obtain a contradiction.

Case $K<\Gamma O^{-}(8, q)$. Since $K^{*}$ is line-transitive but not flag-transitive, it cannot be point-transitive by (5.12).

Let $r$ be a primitive divisor of $q^{2}+1$ (cf. (2.18)). Let $R \in S y l_{r} K$, and set $W=C_{V}(R)$ and $N=N_{K}(R)$. Then $W$ has type $\Omega^{+}(4, q)$, and $N^{W}$ is point-transitive by Sylow's theorem. Thus, $N_{L}^{L}$ is point-transitive for each line $L \subset W$. Now $K$ is $(1,2)$-transitive, and hence $K^{*}$ is point-transitive.

Case $K<\Gamma U(6, q)$. By (2.12), each line-orbit of $K_{E}^{E}$ has length divisible by $\left(q^{6}-1\right) /\left(q^{2}-1\right) d$ (where $\left.d=(q+1,3)\right)$, while each line-orbit of $K^{*}$ has length divisible by $q^{3}+1$. Exclude for the moment the possibility that $q=2$ and $K^{*}$ is monomial but irreducible. By (5.7) and (2.8), $K^{*}$ fixes a nonsingular point, and $K^{*} \unrhd S U(3, q), 3 \cdot A_{7}, P S L(3,2)$ or $S U(3,2)^{\prime \prime}$. In the first two cases, $q^{2}$ or 5 divides $\left|K_{x E}^{E}\right|$, and we find that $K_{E}^{E}$ is flag-transitive.

Assume that $K^{*(\infty)}=\operatorname{PSL}(3,2)$. By $(2.14 \mathrm{iii}), K_{x}$ has a subgroup $S=P S L(3,2)$ such that $\operatorname{dim} C_{V}(S)=3$. An element of $S$ of order 3 fixes a point $y \in[V, S]$ and induces a transvection on $y^{\perp} / y$. This contradicts the known action of $K^{*}$.

This leaves us with the case $q=2$. Note that $K=O^{2}(K) \leqslant G U(6,2)$. Let $R \in \mathrm{Syl}_{7} K$. Then $R$ fixes exactly 2 planes. Since $\left|C_{G U(6,2)}(R)\right|$ is odd, there is an involution $j \in N_{K}(R)$ interchanging these planes. If $v$ is a vector in one of these planes, then $\left(v+v^{j}, v+v^{j}\right)=0$. Thus, $C_{V}(j)$ is a plane, which we may assume is $E$. Set $P=C_{K}(E)$. In view of the known action of $K^{*}, P^{x^{1} / x}$ lies inside of a dihedral group of order 8. On the other hand, a Sylow 7-subgroup $R_{1}$ of $K_{E}$ acts fixed-point freely on $P$. If $|P|=8$, then all nontrivial elements of $P$ are $R_{1}$-conjugate to $j$, and hence $P$ acts faithfully on $x^{\perp} / x$. Thus, we must have $|P|>8$, and hence $|P| \geqslant 64$. If $x \neq y \in x^{R_{1}}$, it follows that $C_{P}\left(x^{\perp} / x\right) \cap C_{P}\left(y^{\perp} / y\right)$ contains an involution $i$. Thus, $\operatorname{dim} C_{V}(i) \geqslant 5$, which is clearly impossible.

Case $K<\Gamma U(7, q)$. By (2.12), each point-orbit of $K^{*}$ has length divisible by $q^{5}+1$. By $(5.8), K^{*} \leqslant \Gamma U\left(1, q^{5}\right)$. The latter group has order $\left(q^{5}+1\right) 10 e$, where $q=p^{e}$, and it contains all scalars in $\Gamma U(5, q)$. Thus, $q^{5}+1$ must be a divisor of $\left(q^{5}+1\right) 10 e /(q+1)$. This is only possible if $q$ is 9 or 4 . However, $\Gamma U\left(1,9^{5}\right)$ has a point-orbit of length $\left(9^{5}+1\right) / 2$. Thus, $q=4$. Since $K=O^{2^{\prime}}(K),\left|K^{*}\right|=\left(4^{5}+1\right) 5$. By (2.1), $\left|K_{x}\right|$ is odd. If $R \in S y l_{7} K$, then $R$ fixes 2 planes, and hence $\left|N_{K}(R)\right|$ is even; but then so is $\left|K_{x}\right|$. This contradiction completes the proof of (5.17).

LEMMA 5.18. If $n=3$, then $\Gamma(V)$ has no subgroup which is $(1,2)$-transitive and has 2 orbits of planes, $(1,3)$-flags and flags.

Proof. Let $K$ be such a subgroup. Let $E$ be a plane, let $x \in E$, and consider both $K_{E}^{E}$ and $K^{*}=K_{x}^{x^{\perp} / x}$. Since $K_{E}^{E}$ is flag-transitive, it contains $S L(E)$, except perhaps 
if $q=2$ or 8 (by (2.7)). On the other hand, $K^{*}$ is a $(1,2,2)$-group, so that (5.13) applies. Use of $K_{x E}^{E E^{x}}$ eliminates $(1,2,2)_{\mathrm{ii}}$. In every other case in (5.13), there is a plane $E$ such that $p||\left(K_{E}^{*}\right)^{E / x} \mid$, and then $K_{E}^{E} \geqslant S L(E)$.

Thus, $K_{x E}^{E} \geqslant A \rtimes S L(2, q)$, where $|A|=q^{2}$. Since $K^{*}$ is irreducible, (2.1) shows that $K^{*}$ must also contain a section $A \rtimes S L(2, q)$. By (5.13), either $K^{*} \unrhd S p\left(2,2^{2}\right)$ or $K^{*} \unrhd Z_{3}^{3} \rtimes A_{4}$, where $K<\Gamma S p(6,2)$ or $\Gamma O^{-}(8,2)$, respectively. In either case, the existence of an $S_{4}$ section in $K^{*}$ forces $\left|\left(K_{E}^{*}\right)^{E / x}\right|$ to be even for every plane $E$ on $x$. But then $K_{E}^{*}$ has an $S_{4}$ section for every $E$ (as above), and this is not the case.

THEOREM 5.19. Let $n=3$, and let $K$ be a point-transitive subgroup of $\Gamma(V)$ having 2 orbits of lines, planes and (1,2)-flags, 3 of (1,3)- and (2,3)-flags, and 4 of flags. Then either

(i) $K \unrhd G_{2}(q)^{\prime}$, embedded naturally in $\Gamma O(7, q)$ or (if $q$ is even) in $\Gamma S p(6, q)$; or

(ii) $K \unrhd \Gamma \operatorname{Sp}\left(2, q^{3}\right), q=2$ or 8 , embedded naturally in $\Gamma \operatorname{Sp}(6, q)($ or $\Gamma O(7, q))$.

Proof. We will proceed in several steps.

(I) There are planes $E$ and $F$ such that $K_{E}^{E}$ is point-transitive while $K_{F}^{F}$ has 2 point-orbits $\Omega, \Omega^{\prime}$ and 2 line-orbits. Each line meeting both $\Omega$ and $\Omega^{\prime}$ produces 2 flag-orbits. Hence, $K_{F}^{F}$ has 3 flag-orbits, $K_{E}^{E}$ is flag-transitive.

By (2.7), $K_{E}^{E} \geqslant S L(3, q)$ except, perhaps, if $q=2$ or 8 and $K_{E}^{E}$ has order $\left(q^{2}+q+1\right)(q+1)$.

Let $x$ be a point. By hypothesis, $K^{*}=K_{x}^{x^{\perp} / x}$ is a $(2,3,4)$-group.

(II) For each line $L, K_{L}^{L}$ is transitive. Suppose that $V$ is unitary. Let $r$ be a primitive divisor of $q^{2}+1$ (cf. (2.18)). An element of order $r$ in $K_{L}$ centralizes $L^{\perp} / L$. Thus, if $L \subset F$ then $r|| K_{L F}^{L} \mid$. Then $K_{F}^{F}$ is line-transitive by Sylow's theorem. Thus, $V$ is not unitary.

Suppose that $q \neq 2, K<\Gamma O(7, q)$ or $\Gamma O^{-}(8, q)$, and $K_{E}^{E} \geqslant S L(3, q)$. Then $C_{K}\left(E^{\perp} / E\right)^{E} \geqslant S L(3, q)$. By $(2.14), K_{E}$ has a subgroup $H$ inducing $S L(3, q)$ on $V / E^{\perp}$ and such that $V / E$ has an $H$-complement $W / E$ to $V / E^{\perp}$. Here, $W$ has type $\Omega^{+}(6, q)$. Passing to $S L(4, q)$ and again applying $(2.14)$, we find that $H$ may even be assumed to fix a second plane of $W$. Now $H$ contains long root groups, and (2.4) applies. Only (2.4 ii) can occur.

(III) Since $F$ contains representatives from both line-orbits, there is a line $L$ contained only in planes from $F^{K}$, and having $K_{L}$ transitive on these planes. We may assume that $L \subset F$, and that $E \cap F=M$ is a line. Note that $K_{M}$ is intransitive on $M^{\perp} / M$.

Suppose that $K<\Gamma O^{-}(8, q)$, where $q=2$ or 8 . Since $q^{2}+1|| K_{L} \mid$, if $D \in \operatorname{Syl}_{5} K$ then $D \neq 1$ and $C_{V}(D)$ has type $\Omega^{+}(4, q)$. Then $N_{K}(D)$ is point-transitive on $C_{V}(D)$, and hence $(q+1)^{2}|| N_{K}(D) \mid$. Since $|D|=5,(q+1)^{2}|| C_{K}(D) \mid$. It follows that $C_{K}(D)$ has an element $r$ of order 3 such that $C_{V}(r)$ has type $\Omega^{+}(6, q)$.

Let $R$ be a 3-group maximal with respect to centralizing a line. Set $W=C_{V}(R)$, $N=N_{K}(R)$ and $C=C_{N}\left(W^{\perp}\right)$. Then $N_{L^{\prime}}^{L^{\prime}}$ is transitive for each line $L^{\prime} \subset W$ (by the Frattini argument). Also, $W$ has type $\Omega^{+}(6, q)$. (If $W$ had type $\Omega^{+}(4, q)$ or $\Omega^{-}(6, q)$ then $R^{W^{\perp}}$ would fix a point.) By $(5.1), N^{W} \geqslant \Omega^{+}(6, q)$ or $A_{7}$. Thus, $C^{W} \geqslant \Omega^{+}(6, q)$ or $A_{7}$. By (2.4), $C^{W} \geqslant A_{7}$ and $q=2$. 
There is a nonsingular vector $w \in W$ such that $C_{W} \geqslant A_{6}$. Let $0 \neq v \in W^{\perp}$, and set $y=\langle v+w\rangle$. Then $K_{y}^{y^{\perp} / y}$ contains a subgroup $A_{6}$ fixing a nonsingular vector. Since $K^{*}$ is a $(2,3,4)$-group, this is impossible.

(IV) From now on, we will assume that $\Gamma<\Gamma \operatorname{Sp}(6, q)$, where $q$ is odd, 2 or 8 .

Suppose that $q$ is odd. There is an involution $t \in K_{M E}$ inducing -1 on $M$. We may assume that $-1 \in K$. Let $R \in S y l_{2} C_{K}(M)$, and define $W, N$ and $C$ as above. Then $\operatorname{dim} W=4$, so $R$ cannot be properly contained in a larger 2-group centralizing a line. Note that $N_{M}^{M} \geqslant G L(2, q)$, while $N_{L^{\prime}}^{L^{\prime}}$ is transitive for any line $L^{\prime} \subset W$ and $N^{W}$ has at most 2 line-orbits. By (5.12), $C^{W}$ is transitive on points. This contradicts the intransitivity of $K^{*}$.

(V) Thus, $q=2$ or 8 .

Next, suppose that $K^{*}$ has an element of order 3 centralizing a point. Let $R$ be a 3-group maximal with respect to centralizing a line, define $W, N$ and $C$ as above, and again find that $C^{W}$ is point-transitive. Thus, $K^{*}$ has no such element.

However, $K^{*}$ is a $(2,3,4)$-group. By $(5.6), K^{*}$ fixes a line. Thus, $K_{x}$ fixes a unique plane $F(x)$ on $x$.

We must distinguish between the cases $F(x) \in E^{K}$ and $F(x) \in F^{K}$.

(VI) If $F(x) \in E^{K}$, note that $F\left(x^{g}\right)=F(x)^{g}=F(x)$ whenever $g \in K_{F(x)}$. Thus, $\left|E^{K}\right|=q^{3}+1$. The cosets of the members of $E^{K}$ form an affine translation plane $Q$ (Dembowski [10, p. 133]).

Since $K^{*}$ is transitive on the $q^{3}$ lines opposite $F(x) / x, \mathcal{Q}$ is desarguesian and $K \leqslant \Gamma L\left(2, q^{3}\right)[\mathbf{1 0}$, pp. $130-131]$. Since $|K|$ is divisible by

$$
q^{3}(q+1) \cdot\left(q^{6}-1\right) /(q-1),
$$

it follows that $K \geqslant \Gamma L\left(2, q^{3}\right)$.

(VII) Now suppose that $F(x) \in F^{K}$. Note that $K_{x}$ is transitive on the lines of $F(x)$ through $x$. If $L^{\prime}$ is such a line, then $\left|L^{\prime K}\right|=\left|x^{K}\right|(q+1) /(q+1)=$ $\left(q^{6}-1\right) /(q-1)$. The points of $V$, together with $L^{\prime K}$, form a generalized hexagon with parameters $q$, $q$. (For, $K^{*}$ has just 2 point-orbits, so all lines in $L^{\prime K}$ through $x$ lie in $F(x)$. If $x_{1}, \ldots, x_{5}$ are distinct points such that $\left\langle x_{i}, x_{i+1}\right\rangle \in L^{\prime K}$ for all $i(\bmod 5)$, then all $x_{i}$ are perpendicular since, for example, $\left\langle x_{1}, x_{2}\right\rangle,\left\langle x_{1}, x_{3}\right\rangle \subset F\left(x_{1}\right)$; thus, all $F\left(x_{i}\right)$ coincide, which is absurd. Thus, there are no triangles, quadrangles or pentagons, and a count shows that we have a generalized hexagon.) By Yanushka $[63,(1.1)]$, this is the $G_{2}(q)$ hexagon.

Since $K$ has just 2 plane-orbits, $\left|E^{K}\right|=q^{3}\left(q^{3}+1\right)$. Then $|K|=q^{3}\left(q^{3}+1\right)\left|K_{E}\right|$. If $q=2$ then $K$ contains a Sylow 3-subgroup of $G_{2}(2)^{\prime} \cong \operatorname{PSU}(3,3)$, and hence $K \unrhd G_{2}(2)^{\prime}$.

Finally, if $q=8$ then we may assume that $K$ does not contain any nontrivial scalars, and hence that $|K|=8^{3}\left(8^{3}+1\right)\left(8^{2}+8+1\right)(8+1)$. Set $H=K \cap G_{2}(8)$. Then $|H|=|K| / 3$ (since $K_{E}^{E} \$ G L(3,8)$ ).

$G_{2}(8)$ has a class of $8^{3}\left(8^{3}-1\right)$ subgroups $S \cong S U(3,8)$. Choose $S$ so that $S \cap H$ contains a Sylow 3-subgroup of $H$. Note that $|S \cap H| \geqslant|H| / 8^{3}\left(8^{3}-1\right)=$ $\left(8^{3}+1\right) 3 / 7>2(8+1)^{2}$. Then $S \cap H=S$ by Hartley [22], and (2.4) yields the desired contradiction in this case. This completes the proof of (5.19). 
REMARK 5.20. (i) In its natural embedding in $\Gamma S p(6, q)$ with $q=2$ or 8 , the group $K=\Gamma L(2, q)=\Gamma \operatorname{Sp}(2, q)$ actually satisfies the hypotheses of (5.19). Indeed, $K$ produces a partition $E^{K}$ as in (VII), consisting of planes since isotropic 1-spaces over $G F\left(q^{3}\right)$ have dimension 3 over $G F(q)$. Also, $\left|K_{E}\right|=q^{3}\left(q^{3}-1\right)(q+1)$ and $\left|K_{E}^{E}\right|=\left(q^{3}-1\right)(q+1)$, where $K_{E}^{E}$ is flag-transitive. Let $K^{*}$ be the group induced by $K_{x}$ on $x^{\perp} / x$. Certainly $K^{*}$ stabilizes $E / x$, and permutes its points transitively. Since $O_{2}\left(K_{x}\right)$ is transitive on $E^{K}-\{E\}, K^{*}$ is transitive on the lines missing $E / x$. A subgroup of $K^{*}$ of order $q+1$ fixes a line missing $E / x$ and acts transitively on that line. Thus, $K^{*}$ has 2 orbits on points, 3 on lines and 4 on flags. Then $K$ has just 2 line-orbits. Finally, if a plane meets $E$ in a line, it meets each other plane in $E^{K}$ in at most one point, so $K$ has 2 plane-orbits.

However, the permutation representation of $\Gamma S p(6, q)$ on the set $X$ of cosets of $\Gamma L\left(2, q^{3}\right)$ does not have rank 3 . For, if it did, the permutation character would be $1+\chi+\zeta$ with $\chi=(1 \mid 2)$, by Table II of $\S 3$. However, $|X|-1-\chi(1)$ does not divide $|\Gamma \operatorname{Sp}(6, q)|$.

(ii) The groups $K=G_{2}(q)^{\prime}$ also satisfy the hypotheses of (5.19), since $K_{x}$ behaves as indicated in (VII).

It is known that $S p(6,2)$ has rank 3 on its class of subgroups $G_{2}(2)$ (Frame [19], Edge [15]). In view of (1.1), we must consider the rank for other values of $q$ as well.

The group $\Omega(7, q)$ has 2 classes of subgroups $G_{2}(q)$, which are all in a single class under $\Gamma O(7, q)$. These can be viewed as follows. Consider $\Omega^{+}(8, q)$, with underlying vector space $V$. There is a subgroup $G$ of $\Omega^{+}(8, q)$ such that $-1 \in G$, $G /\langle-1\rangle \cong \Omega(7, q)$, and $G^{*}=(G /\langle-1\rangle)^{\tau}$ fixes a nonsingular point $b$, where $\tau$ is a triality automorphism of $P \Omega^{+}(8, q)$. Since $G^{*}$ is transitive on each class of 4-spaces, $G$ is point-transitive. Moreover, $G^{*} \cap G^{* \tau^{-1}}=C_{G^{*}}(\tau)=G_{2}(q)$. Note that $b$ can be chosen to be in any $\Omega^{+}(8, q)$-orbit of nonsingular points of $V$. There are $(2, q-1)$ such orbits, conjugate in $N_{\Gamma O^{+}(8, q)}(G)$. Checking orders, we find that $G$ is transitive on each class $X$ of nonsingular points of $V$.

If $b, c \in X, b \neq c$, then $\langle b, c\rangle$ may be anisotropic or contain a unique singular point; it can also contain 2 singular points if $q>3$. Thus, if $q>3$ then $G$ cannot have rank 3 on $X$.

Suppose that $q=3$. If $\langle b, c\rangle$ is anisotropic, then $G_{b c}=S U(3,3) \cdot 2$. If $\langle b, c\rangle$ contains a singular point $r$, then $G_{b r}$ is a parabolic subgroup of $G_{b}=G_{2}(3)$, has an element inverting $r$, and hence acts nontrivially on $\langle b, c\rangle$. Since $G_{b}$ is transitive on the points of $b^{\perp}$, it is transitive on $\{c \in X \mid\langle b, c\rangle$ is an anisotropic 2-space $\}$. Consequently, $G$ has rank 3 on $X$. (Of course, the case $q=2$ can be handled similarly.)

(D) Subgroups of $\mathrm{\Gamma O}^{+}(8, q)$. The case of $\mathrm{\Gamma O}^{+}(8, q)$ is somewhat similar to the rank 3 cases already considered. The only interesting result is (5.22), which deals with $W\left(E_{8}\right)^{\prime}$. We begin with an analogue of (5.15).

LEMMA 5.21. If $K$ is a subgroup of $\mathrm{TO}^{+}(8, q)$ which is transitive on both points and lines, then $K \geqslant \Omega^{+}(8, q)$. 
Proof. By (2.12), $K_{x}^{x^{\perp} / x}$ is either point-transitive or has 2 point-orbits of length $\frac{1}{2}\left(q^{2}+1\right)\left(q^{2}+q+1\right)$. By (5.1), $K_{x}$ is flag-transitive, and hence (2.6) applies.

Proposition 5.22. Let $K<\Gamma \mathrm{O}^{+}(8, q)$, and assume that $K$ stabilizes each class of 4-spaces, is transitive on each class of (1,4)-flags, has 2 orbits of lines, (1,2)-flags, and each class of $(2,4)-,(1,2,4)$ - and $(1,3)$-flags, and has 3 orbits of flags. Then $K$ is $W\left(E_{8}\right)^{\prime}$, embedded naturally in $\Omega^{+}(8,3)$.

Proof. Set $K^{*}=K_{x}^{x^{\perp} / x}, A(x)=C_{K}(x) \cap C_{K}\left(x^{\perp} / x\right)$ and $V(x)=[V, A(x)]$. By hypothesis, $K^{*}$ is transitive on each class of planes, and has 2 orbits of points, lines and $(1,3)$-flags and 3 orbits of flags. By $(5.1), K^{*(\infty)}=\Omega^{-}(4, q)$ or $\Omega(5, q)^{\prime}$, embedded naturally in $\Omega^{+}(6, q)$.

Case $K^{*(\infty)}=\Omega^{-}(4, q)$. Let $F$ be a 4 -space. By $(5.1),\left(K_{F}^{F}\right)^{(\infty)}=S p(4, q)^{\prime}$ or $S L\left(2, q^{2}\right)$. Computing $|K|$ using points or a class of 4 -spaces, we find that $\left(K_{F}^{F}\right)^{(\infty)}=S L\left(2, q^{2}\right)$.

Suppose that $A(x) \neq 1$. By $(2.1,4), V(x) / x$ is anisotropic. If $x \in F$ then $F \not \subset V(x)^{\perp}$. Thus, $A(x)^{F}$ is nontrivial and contains transvections, which is not the case.

Consequently, $A(x)=1$ and $K_{x}^{(\infty)} \cong \Omega^{-}(4, q)$. If $q \neq 3$, then $K_{x}^{(\infty)}$ centralizes a 4-dimensional space, by (2.14); but then $A(y) \neq 1$ for some point $y$.

Thus, $q=3$ and $\operatorname{dim} W(x)=3$, where $W(x)=C_{V}\left(K_{x}^{(\infty)}\right)$. Here, $W(x) / x$ is anisotropic.

Let $f \in K_{x}$ have order 5. Then $\operatorname{dim} C_{V}(f)=4$, and $f$ centralizes $W(y)$ for each $y \in C_{V}(f)$. Thus, $T=W(x) \cap W(y)$ is an anisotropic 2-space if $y \in C_{V}(f)$ but $y \neq x$. Then $H=\left\langle K_{x}^{(\infty)}, K_{y}^{(\infty)}\right\rangle$ acts on $T^{\perp}$. By $(5.7), H$ is $2 \cdot \operatorname{PSL}(3,4)$. Set $\langle j\rangle=Z(H)$.

Let $T_{1}$ be a second anisotropic 2-space in $W(x)$, and let $y_{1}$ denote the point other than $x$ in $T_{1}^{\perp} \cap C_{V}(f)$. This produces a second group $2 \cdot \operatorname{PSL}(3,4)$, and a second involution $j_{1}$. Both $j$ and $j_{1}$ invert $W(x)^{\perp}$, and both centralize $W(x) / x$. Hence $j j_{1} \in A(x)=1$, and $K^{*(\infty)}$ cannot be $\Omega^{-}(4, q)$.

Case $K^{*(\infty)}=\Omega(5, q)^{\prime}$. If $q>3$, then (2.14) applies: $K_{x}$ has a subgroup $\Omega(5, q)$ centralizing a 3 -dimensional subspace. Then (2.4) yields a contradiction.

Suppose that $q=3$. Then $K_{x}^{(\infty)}$ has a subgroup $J=Z_{2}^{4} \rtimes A_{5}$, where $\operatorname{dim} C_{V}\left(O_{2}(J)\right)=3$. Thus, $\operatorname{dim} C_{V}(J)=3$, and $J$ acts monomially on $[V, J]$. In particular, $A(y) \neq 1$ for some $y$, so that $|A(y)|=3$ and $\operatorname{dim} V(y)=2$ by $(2.1,4)$ and the action of $K^{*}$. Moreover, there are points $y, z \in[V, J]$ such that $V(y) \cap V(z)$ is a nonsingular point $b$.

Since $K_{y}^{(\infty)}$ centralizes $V(y) / y$, it centralizes $V(y)$. Then $K_{b} \geqslant\left\langle K_{y}^{(\infty)}, K_{z}^{(\infty)}\right\rangle$, where $K_{y}^{(\infty)} \neq K_{z}^{(\infty)}$ (as otherwise $K_{y}^{(\infty)}$ would act on the nonsingular 3-space $\langle b, y, z\rangle$, in which case $|A(y)|$ would be at least $\left.3^{3}\right)$. By (2.16), $K_{b}^{b^{\perp}} \geqslant W\left(E_{7}\right)^{\prime}$.

Note that $A(y)$ moves $b$ to another nonsingular point $c$ in $V(y)$. Then $K_{c}^{c^{\perp}} \geqslant W\left(E_{7}\right)^{\prime}$. Moreover, $\left(K_{y}^{(\infty)}\right)^{b^{\perp}}$ lies in a unique subgroup $W\left(E_{7}\right)^{\prime}$ (see the proof of (2.16), Case 8); a similar statement holds for $\left(K_{c}^{(\infty)}\right)^{c^{\perp}}$. Thus, $\left\langle K_{b}^{(\infty)}, K_{c}^{(\infty)}\right\rangle$ is contained in a group $W\left(E_{8}\right)^{\prime}$, and hence is $W\left(E_{8}\right)^{\prime}$. Also, $b^{K}$ contains all "roots" for the latter group. Consequently, $K=W\left(E_{8}\right)^{\prime}$, as desired. 
Finally, if $q=2$ then $K^{*}>\Omega^{-}(4,2)$. By $(2.14), K_{x}$ has a subgroup $\Omega^{-}(4,2)$ centralizing a 4-dimensional subspace. This time, $|A(x)| \geqslant 4$ in view of the action of this $\Omega^{-}(4,2)$, and $(2.1,4)$ yield a contradiction.

LEMMA 5.23. $\mathrm{TO}^{+}(8, q)$ has no subgroup stabilizing each class of 4-spaces, transitive on points and each class of 4-spaces, and having 2 orbits on lines, planes and each class of $(1,4)$-flags, 3 orbits on (1,2)-flags and each class of $(2,4)$-flags, 5 orbits on $(1,3)$ and (2,3)-flags and each class of $(1,2,4)$-flags, and 9 orbits of flags.

Proof. Let $K$ be such a group. Let $F$ be a 4-space, and set $H=K_{F}^{F}$. Then $H$ has 2 point-orbits, 3 line-orbits, 5 orbits of (1,2)-flags and 9 flag-orbits. Corresponding statements hold for $K^{*}=K_{x}^{x^{1} / x}$. (In fact, our hypotheses are invariant under triality.)

First consider the possibility that $H^{(\infty)}=\Omega^{-}(4, q) \cong K^{*(\infty)}$. If $q$ is odd, then $K^{*(\infty)}$ is $\Omega\left(3, q^{2}\right)$, in its natural embedding into $\Omega^{+}(6, q)$. By (2.1), (2.4) and (2.14), $K_{x}^{(\infty)}$ centralizes a hyperbolic line. If $P \in S y l_{p}\left(K_{x} \cap \Omega^{+}(8, q)\right)$, then $\operatorname{dim} C_{V}(P)=4$, $\operatorname{dim} \operatorname{rad} C_{V}(P)=2$, and $N_{K}(P)$ cannot be transitive on the fixed points of $P$.

If $q$ is even, then $K^{*(\infty)}$ fixes a nonsingular 1 -space $b$, and induces at least $S L\left(2, q^{2}\right)$ on $b^{\perp} / b$. By (2.1) and (2.4), $A(x)=C_{K}(x) \cap C_{K}\left(x^{\perp} / x\right)$ has order at most $q$. Let $x \neq y \in C_{V}(A(x))$. Then $A(x)^{y^{y^{\perp} / y}}$ lies inside an $S L\left(2, q^{2}\right)$, and hence has order 1 or at least $q^{2}$. Consequently, $A(x)=1$. By $(2.14), H^{1}\left(K^{*(\infty)}, x^{\perp} / x\right)=0$. Thus, $K_{x}^{(\infty)}$ again centralizes a hyperbolic line, and we obtain the same contradiction as before.

It remains for us to show that there are no possibilities for $H^{(\infty)}$ other than $\Omega^{-}(4, q)$. This involves checking all the possibilities in (5.1). Most are eliminated by the following facts.

$H$ has 2 point-orbits.

There is a line $L \subset F$ such that $H_{L}^{L}$ is transitive.

There is a plane $E$ such that $H_{E}^{E}$ fixes a point, a line, or (if $q=4$ ) a hyperoval; moreover, $H_{E}^{E}$ contains either an elementary abelian group of order $q^{2}$ or $A_{6}$. (For, let $E^{H}$ and $E^{\prime H}$ be the plane-orbits of $H$, where $H_{E}^{E}$ has fewer point-orbits than $H_{E^{\prime}}^{E^{\prime}}$. If $H_{E}^{E}$ has 2 point-orbits, then it has at least 3 flag-orbits; by (2.7 iii), $H_{E^{\prime}}^{E^{\prime}}$ has at least 6 flag-orbits, and hence $H_{E}^{E}$ behaves as desired. If $H_{E}^{E}$ is transitive let $\Omega_{1}, \Omega_{2}, \Omega_{3}, \Omega_{4}$ be the point-orbits of $H_{E^{\prime}}^{E^{\prime}}$. If no line of $E^{\prime}$ meets all orbits $\Omega_{i}$, then joining the $\Omega_{i}$ in pairs accounts for at least 9 flag-orbits, and leaves $H_{E}^{E}$ with none. Thus, some line meets $\Omega_{i}$, and accounts for 4 flag-orbits. This leaves only 4 flag-orbits for the remaining 3 line-orbits of $K_{E^{\prime}}^{E^{\prime}}$, and (2.7 iv) yields a contradiction.)

Applying (5.1) we find only one situation requiring further comment: that in which $H$ fixes a line $L$. Here, $H$ is transitive on $L$, on the $q^{2}(q+1)$ points $y \notin L$, on the $q(q+1)^{2}$ lines meeting $L$ in a point, and on the $q^{4}$ lines skew to $L$. As in (2.12), $H_{y}$ has just 2 orbits of lines on $y$. The same holds for $H_{x}, x \in L$. Then $H$ has only 4 orbits of $(1,2)$-flags, and this situation cannot occur.

Lemma 5.24. $\mathrm{\Gamma O}^{+}(8, q)$ has no subgroup stabilizing each class of 4-spaces and having 2 orbits on points and each class of 4-spaces, 4 orbits on planes and each type of (1,4)-flags, 5 orbits on (1,2)-flags and each type of $(2,4)$-flags, 7 orbits on $(1,3)$-flags and each type of $(1,2,4)$-flags, and 10 orbits of flags. 
Proof. Let $K$ be such a subgroup. As in the proof of (5.23), if $E$ is a plane such that $K_{E}^{E}$ has $i$ orbits of points and lines, then $K_{E}^{E}$ has at least $2 i-1$ flag-orbits. The only partitions of 7 with 4 parts are

$$
7=4+1+1+1=3+2+1+1=2+2+2+1 .
$$

Since there are only 10 flag-orbits, it follows that $K_{E}^{E}$ is flag-transitive for some plane E.

Let $F$ be a 4 -space containing $E$. By $(2.15), K_{F}^{F}$ contains $S L(4, q)$ or $A_{7}$, or else fixes a point or a plane.

If $K_{F}^{F}$ contains $S L(4, q)$ or $A_{7}$, let $\tau$ denote a triality automorphism of $P \Omega^{+}(8, q)$. Then (2.16) applies to $(K / Z(K))^{\tau}$; but then $K$ either fixes $F$ or contains $\Omega^{+}(8, q)$ or $A_{9}$, and hence cannot satisfy the required orbit conditions.

Thus, $K_{F}^{F}$ fixes a point or a plane. If $K_{F}^{F}$ fixes both a point and a plane, then it has at least 10 flag-orbits, which is impossible. Thus, $K_{F}^{F}$ has 2 orbits of points and lines, and 3 orbits of $(1,2)$-flags.

Let $F^{\prime} \in F^{\Omega^{+}(8, q)}-F^{K}$. Then $K_{F^{\prime}}^{F^{\prime}}$ has 2 orbits of points, lines and $(1,2)$-flags. Then $K_{L F^{\prime}}^{L}$ is transitive for each line $L$ of $F^{\prime}$, and hence $K_{F^{\prime}}^{F^{\prime}}$ has only one point-orbit, which is not the case.

LEMMA 5.25. $\mathrm{\Gamma O}^{+}(8, q)$ has no point-transitive subgroup having 2 plane-orbits and 3 orbits of (1,3)- and (1,4)-flags.

Proof. Let $K$ be such a subgroup. There is a plane $E$ such that $K_{E}^{E}$ is transitive. By (2.7), if $F$ is a 4-space containing $E$ then $K_{E F}^{E}$ is still transitive, as is $K_{x E}^{E}$ for some point $x$ of $F$. By (2.15), $K_{x}^{x^{1} / x}$ fixes a plane or a pair of planes. In either case $K_{x}^{x^{\perp} / x}$ has more than 3 plane-orbits, contrary to hypothesis.

6. Subspace transitivity. For rank $n \geqslant 4$, our study of highly transitive subgroups of $\Gamma(V)$ will employ the elegant method of Perin [46]. This method was, in fact, already used several times in $\S 5 \mathrm{C}$. As in $\S 5$, we will require numerous technical results. The only general results are $(6.1,6.4,6.5)$.

The following notation will be used throughout this section, as well as in $\S 12$.

$K \leqslant \Gamma L(V)$, with $K$ point-transitive.

$x$ is a point.

$r$ is a prime and $R$ is an $r$-subgroup of $K_{x}$.

$W=C_{V}(R), N=N_{K}(R)$ and $C=C_{N}\left(W^{\perp}\right)$.

Usually, $R \in S y l_{r} K_{x}$. Then, by Sylow's theorem, $N^{W}$ is point-transitive. Perin's method involves comparing $N^{W}$ and $N^{W^{\perp}}$ in order to show that $C^{W}$ is relatively large. We begin with a simple example.

THEOREM 6.1. Let $K$ be a point-transitive subgroup of $\Gamma U(d, q)$, where $d \geqslant 4$. Assume that $K$ is transitive on lines; if $d=6$ or 7 , assume further that $K$ is also $(1,2)$-transitive. Then $K \geqslant S U(d, q)$.

Proof. By (5.12), we may assume that $d \geqslant 6$. Let $r$ be a primitive divisor of $q^{d-3}+(-1)^{d}$ (cf. $\left.(2.18)\right)$; we will exclude the cases $\Gamma U(6,2)$ and $\Gamma U(9,2)$ until the end of the proof. Let $R \in S y l_{r} K_{x}$. By Table IV, $R \neq 1$. Since $r$ divides the number of nonzero vectors of $W^{\perp}$, from Table IV it also follows that $W$ is a nonsingular 3-space. 


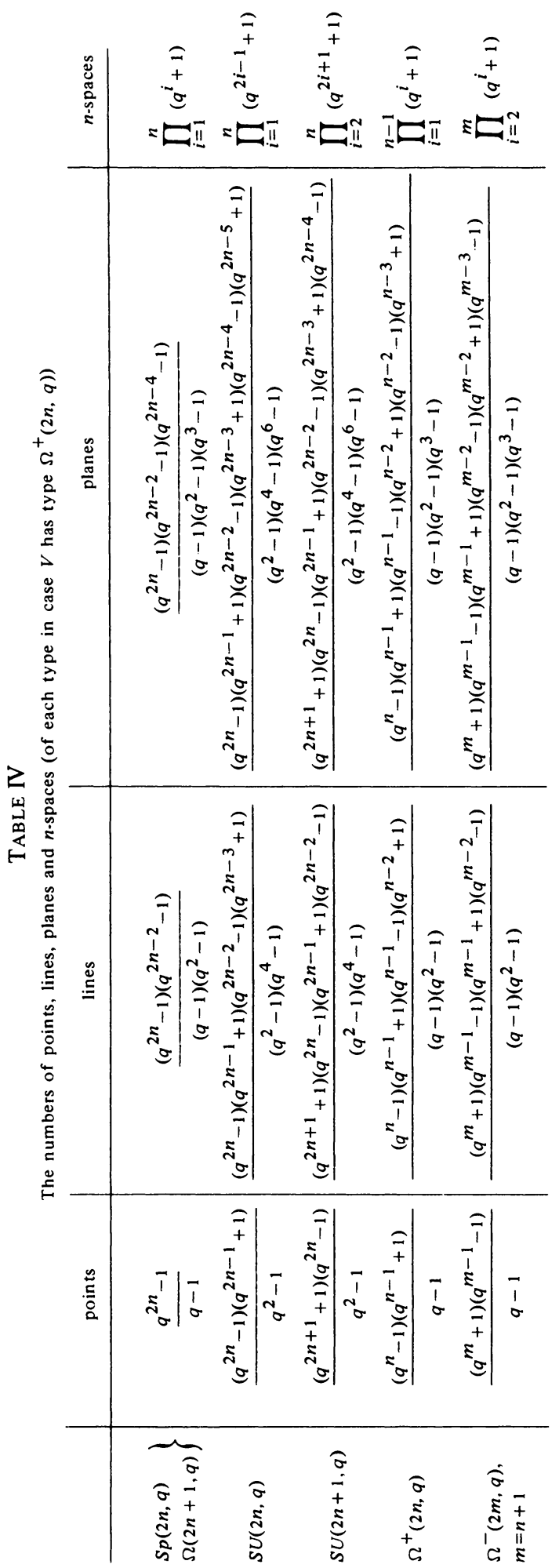


By (2.12), $N^{W} \unrhd S U(3, q), P S L(3,2), 3 \cdot A_{7}$, or an extraspecial group of order 27. Also, $N^{W^{\perp}}$ is a subgroup of $\Gamma L\left(2, q^{d-3}\right)$.

If $N^{W}$ contains $S U(3, q), P S L(3,2)$ or $3 \cdot A_{7}$, then so does $C^{W}$. If $C^{W} \geqslant S U(3, q)$, then (2.4) applies.

We claim that $K_{x}$ acts irreducibly on $x^{\perp} / x$. This is clear if $d=6$ or 7. If $d \geqslant 8$, then $\left|K_{x}\right|$ is divisible by primitive divisors of $q^{d-2}-(-1)^{d}$ and $q^{d-3}+(-1)^{d}$, from which irreducibility follows immediately. (If $K<\Gamma U(8,2)$, this argument fails. However, in this case $K$ is $(1,2)$-transitive by (2.12), and then the irreducibility of $K_{x}$ is obvious.)

In particular, by (2.1) and (2.4), we may assume that $C \cap Q=1$, where $Q=O_{p}\left(S U(V)_{x}\right)$. But in both of the exceptional cases $q=3$ and 5, $C^{W} \cap Q^{W} \neq 1$. Thus, we are left with the case $q=2$.

Now all we know is that $N^{W}$ has a normal extraspecial subgroup of order 27 and exponent 3. Since $N^{W^{\perp}} \leqslant \Gamma L\left(2,2^{d-3}\right)$, it follows that $C^{W}$ contains the center $\langle t\rangle$ of $S U(3,2)$. Note that $t$ centralizes the hyperplane $\left(W^{\perp}+x\right) / x$ of $x^{\perp} / x$. Thus, Wagner [60] applies to the group $H$ induced by $K_{x}$ on $x^{\perp} / x$. Using $r$, we find that $H \geqslant S U(d-2,2)$. Consequently (2.6) applies.

Finally, suppose that $K \leqslant \Gamma U(6,2)$ or $\Gamma U(9,2)$. In the former case, $K_{x}^{x^{\perp} / x} \geqslant S U(4,2)$ by (5.12). Consider the case $\Gamma U(9,2)$. By $(2.12), K$ is (1,2)-transitive. Let $R \in S y l_{7} K_{x}$, and define $W, N$ and $C$ as before. Then $N^{W}$ is transitive on points, and $\operatorname{dim} W=3$ or 6 . If $\operatorname{dim} W=3$, we can proceed as before. If $\operatorname{dim} W=6$, then $N^{W}$ is $(1,2)$-transitive, so that $N^{W} \geqslant S U(6,2)$. Then $C^{W} \geqslant S U(6,2)$, and (2.4) completes the proof of (6.1).

REMARK. By Table II and (4.2), a subgroup of $\Gamma U(m, q), m \geqslant 6$, is point-transitive if it is line-transitive.

Lemma 6.2. Suppose that $K$ is a point-transitive subgroup of $\Gamma(V)$ which is either transitive on lines or has 2 line-orbits and at most 3 orbits of $(1,2)$-flags. (Moreover, if there are 2 line-orbits and $V$ has type $\Gamma O(2 m+1,3)$ or $\Gamma O^{-}(2 m, 2)$, assume that $K$ has 2 plane-orbits and at most 4 orbits of $(2,3)$-flags.) Define $s$ by the following table.

\begin{tabular}{c|c|c|c|c|c} 
Type of $V$ & $S p(2 m, q)$ & $\Omega(2 m+1, q)$ & $\Omega^{ \pm}(2 m, q)$ & $S U(2 m, q)$ & $S U(2 m+1, q)$ \\
\hline$s$ & $q^{m-2}+1$, & $q^{m-2}+1$, & $q^{m-3} \pm 1($ either sign $)$, & $q^{2 m-5}+1$, & $q^{2 m-3}+1$, \\
& $m \geqslant 4$ & $m \geqslant 4$ & $m \geqslant 6$ & $m \geqslant 4$ & $m \geqslant 4$ \\
& & & $q^{2}+1, m=5$ & &
\end{tabular}

Assume that, for some line $L,\left|K_{L}\right|$ is divisible by a primitive divisor $r$ of $s$. Then $K \geqslant \operatorname{Chev}(V)$.

REMARK 6.3. (i) The parenthetical hypothesis in (6.2) is only used in cases (h) and (j) of the proof.

(ii) Several cases are automatically excluded in (6.2): those in which $s$ has no primitive divisor. For future reference, these excluded cases are listed in the following continuation of the preceding table

Type of $V|S p(10,2)| \Omega(11,2)\left|\Omega^{ \pm}(12,2), \Omega^{ \pm}(18,2)\right| S U(8,2) \mid$ none 
Proof OF 6.2. The argument falls into several steps.

(I) Let $R \in S y l_{r} K_{L}$. In each case, $W=C_{V}(R)$ is a nonsingular subspace; its type is given in the following continuation of the previous tables.

$$
\text { Type of } W|S p(4, q)| \Omega(5, q)\left|\Omega^{ \pm}(6, q)\right| S U(5, q) \mid S U(4, q) .
$$

Clearly, $R \in S y l_{r} K_{x L}$ for $x \in L$. Also, $r \nmid\left|\Gamma(W)_{x}\right|$ except when $W$ has type $\Omega^{-}(6, q)$ and $V$ has type $\Omega^{-}(10, q)$. Thus, if $R \notin S y l_{r} K_{x}$ then $W$ has type $\Omega^{-}(6, q)$, and $r|| N_{y}^{W} \mid$ for each point $y$ of $W$, in which case (5.10) applies. On the other hand, if $R \in S y l_{r} K_{x}$ then $N^{W}$ is point-transitive. In any event, $N^{W}$ has at most 2 line-orbits and at most 3 orbits of $(1,2)$-flags, by Sylow's theorem. Note that if $N^{W}$ has 2 line-orbits then so does $K$.

In view of $(5.12,5.13,5.15)$, one of the following holds (and is handled in the step indicated):

(a) $N^{W} \geqslant \operatorname{Chev}(W)$ (II);

(b) $N^{W} \unrhd S U(3, q)$ for $W$ of type $\Omega^{-}(6, q)$ (II);

(c) $N^{W} \unrhd S L\left(2, q^{2}\right)$ for $W$ of type $S p(4, q)(\mathrm{V})$;

(c) $N^{W} \unrhd S L(2,5)$ for $W$ of type $S p(4,3)(\mathrm{V})$;

(d) $N^{W}$ is $A_{6}$, for $W$ of type $S p(4,2)(\mathrm{VI})$;

(e) $N^{W}$ is $A_{7}$ or $S_{7}$, for $W$ of type $\Omega^{+}(6,2)(\mathrm{VI})$;

(f) $N^{W} \unrhd 4 \cdot P S L(3,4)$ for $W$ of type $S U(4,3)$ (VII);

(g) $N^{W} \unrhd 2 \cdot P S L(3,4)$ for $W$ of type $\Omega^{-}(6,3)$ (VIII);

(h) $O_{2}\left(N^{W}\right)$ is elementary abelian of order 16 or 32 , and 20||$N^{W} / O_{2}\left(N^{W}\right) \mid$, for $W$ of type $\Omega(5,3)$ (IX);

(i) $O_{2}\left(N^{W}\right)$ is extraspecial of order 32 , and 5||$N^{W} \mid$, for $W$ of type $\operatorname{Sp}(4,3)(\mathrm{X})$; or

(j) $\left|O_{3}\left(N^{W}\right)\right| \geqslant 27$, for $W$ of type $\Omega^{-}(6,2)(\mathrm{XI})$.

(II) By (2.4), we may assume that $K$ contains no long root elements. (In (2.4 iv), there are 3 line-orbits, 2 of which arise from lines of the unitary geometry.)

If $s=q^{i} \pm 1$, then the normalizer of $R^{W^{\perp}}$ in $\Gamma\left(W^{\perp}\right)$ is contained in $\Gamma L\left(2, q^{i}\right)$. Consequently, this statement holds for $N^{W^{\perp}}$. However, $N^{W}$ is not contained in any $\Gamma L\left(2, q^{i}\right)$.

If (a) or (b) holds (excluding $S p(4,2)$ and $S U(3,2)$ ), then $\left(N^{W}\right)^{\prime \prime}$ has no homomorphic image in any $\Gamma L\left(2, q^{i}\right)$, so that $\left(C^{W}\right)^{\prime \prime}=\left(N^{W}\right)^{\prime \prime}$ and $C$ contains long root elements.

If $N^{W} \unrhd S U(3,2)$, then $C^{W}$ contains a transvection (since $\Gamma L\left(2,2^{i}\right)$ never has a quaternion subgroup). Once again, $C$ contains long root elements.

Finally, if $N^{W}$ is $S p(4,2)$, or if one of (c)-(g) holds for $N^{W}$, then one of (c)-(g) also holds for $C^{W}$ in place of $N^{W}$. We will have to consider these possibilities for $C^{W}$ separately. Note that, if (h) or (i) holds for $N^{W}$, it might not hold for $C^{W}$ : only $\left|O_{2}\left(C^{W}\right)\right|=16$ or 32 is clear at this point. Similarly, if (j) holds for $N^{W}$ it need not hold for $C^{W}$.

(III) Set $Q=O_{p}\left(\Gamma(V)_{x}\right), A(x)=K \cap Q$ and $V(x)=[V, A(x)]$. Clearly, $K_{x}$ acts on both $A(x)$ and $V(x)$. Recall that $A(x)$ is assumed to contain no long root 
elements. However, in several of our cases, $C \cap A(x)$ is nontrivial:

(c) $|C \cap A(x)|=q$ and $V(x)$ is a line (since a Sylow $p$-subgroup of $C_{x}$ has order $q^{2}$ and acts on the $q+1$ lines through $x$, centralizing one of these lines: $V(x)$ );

(c') $|C \cap A(x)|=q$ and $V(x)$ is a line;

(d) $|C \cap A(x)|=4$ and $V(x) / x$ is an anisotropic 2-space when $K$ is regarded as lying in $O(2 n+1,2)$ (since $W$ is an obvious section of the standard mod 2 permutation module for $A_{6}$ );

(e) $|C \cap A(x)|=4$ and $V(x) / x$ is again an anisotropic 2-space;

(f) $|C \cap A(x)|=9$ and $V(x)=W \cap x^{\perp}$;

(h) If 3||$N^{W} \mid$ then $|C \cap A(x)|=3$ and $V(x) / x$ is a nonsingular 1-space;

(j) $|C \cap A(x)| \leqslant 2$.

In cases (g) and (i), $C \cap A(x)=1$. In all cases, we will use (2.1) in order to show that $|A(x)|$ is small.

(IV) We claim that $A(x) \leqslant C$. For, suppose not. Then $A(x) \$ C_{K}(R)$. In particular, $|A(x)|>q^{2}$. By (2.1), $V$ is neither symplectic of characteristic 2 nor orthogonal.

If $V$ is of type $S p(2 m, q)$, then $r \mid q^{m-2}+1$, so $|A(x)| \geqslant q^{2 m-4}$. By (2.1), $A(x)$ is abelian and $A(x) Q^{\prime} / Q^{\prime}$ contains $q^{2 m-4}$ mutually orthogonal vectors in a $2 m-2$-dimensional symplectic space. This is clearly impossible.

Thus, $V$ is of type $S U(d, 3)$. By hypothesis, $s=3^{d-4}+1$ or $3^{d-5}+1$. Thus, $|A(x)| \geqslant 3^{2 d-10}$. But $\left|Q / Q^{\prime}\right|=3^{2(d-2)}$ and $2 d-10>d-2$ if $d>8$. Consequently, (2.1) produces the same contradiction as above if $d>8$. This leaves us with the case of $V$ of type $S U(8,3)$. Here, $|A(x)|=3^{6}$ by the above argument. However, $|C \cap A(x)|=3^{2}$ in case (f), whereas our group $R$ of order 7 cannot act fixed-point-freely on a group of order $3^{4}$.

This contradiction proves our claim. In particular, $A(x)=1$ in cases (g) and (i).

(V) In (c) and (c'), use of $N^{W}$ shows that $V(x)=V\left(x^{\prime}\right)$ whenever $x^{\prime} \in V(x)$. Let $L$ be a line on $x$ such that $L \subset V(x)^{\perp}$ but $L \neq V(x)$. If $y \in L$ and $y \neq x$, then $y \in V(x)^{\perp}$ implies that $A(x)$ acts on $V(y)$. Since $V(x) \cap V(y)=0$ and $V(x)=[V, A(x)]$, it follows that $V(y) \subset V(x)^{\perp} \subset x^{\perp}$. Thus, $L=\langle x, y\rangle \subset V(y)^{\perp}$.

Consequently, there are at least 3 types of lines: those of the form $V(x)$, those lines $L$ contained in $V(x)^{\perp}$ for all $x \in L$ (but not of the form $V(x)$ ), and all other lines. Since $K$ has at most 2 line-orbits, this is impossible.

(VI) We treat cases (d) and (e) together. Recall that $|A(x)|=4$ and $V(x) / x$ is an anisotropic 2-space. We first show that, if $y \in V(x)^{\perp}$, then $V(y) \subseteq V(x)^{\perp}$. For, let $B(x)$ be the subgroup of $C$ such that $A(x) \nless B(x) \cong A_{4}$ and $C_{V}(B(x))=C_{V}(A(x))=V(x)^{\perp}$. Then $B(x)$ acts on $A(y)$, and hence $A(x)$ centralizes $A(y)$. In particular, $A(x)$ acts on $V(y)$, fixing each subspace through $y$. If $v \in V(y), a \in A$ and $v^{a} \neq v$, then $v^{a}-v$ must be the third nonzero vector in $\left\langle v, v^{a}\right\rangle$, and hence is in $y \cap V(x)$. Thus, if $y \neq x$ then $A(x)$ centralizes $V(y)$, and hence $V(y) \subset V(x)^{\perp}$.

Let $M$ be a line on $x$ such that $M \varnothing V(x)^{\perp}$. Then $A(x)^{M} \neq 1$. If $x \neq y \in M$ then $y \notin V(x)^{\perp}$, so $x \notin V(y)^{\perp}$ by the preceding paragraph, and hence $A(y)^{M} \neq 1$. Thus, $K_{M}^{M}$ is transitive. 
Since $K$ has at most 2 line-orbits, it has 2 such orbits: those lines $M$ as above, and all remaining lines. In particular, $K$ is transitive on the $(1,2)$-flags of the form $(x ; M)$ with $M \varnothing V(x)^{\perp}$. Then $K_{x}$ is transitive on the points on $x^{\perp} / x$ not in $V(x)^{\perp} / x$. Consequently, $K_{x}$ is transitive on the nonsingular vectors in $V(x)^{\perp} / x$. In particular, $\operatorname{rad} V=0$.

Fix $x$, let $x \neq y \in V(x)^{\perp}$, and set $V(\langle x, y\rangle)=\left\langle V(y), V\left(y^{\prime}\right)\right\rangle$, where $y^{\prime}$ is the third point of $\langle x, y\rangle$. Since $V(y)$ and $V\left(y^{\prime}\right)$ are perpendicular, $V(\langle x, y\rangle)$ is a 6-dimensional subspace of $V(x)^{\perp}$, and $V(\langle x, y\rangle) /\langle x, y\rangle$ has type $\Omega^{+}(4,2)$.

Consider the pairs $(\langle x, y\rangle,\langle x, d\rangle)$ with $y \in V(x)^{\perp}, d \in V(\langle x, y\rangle)$, and $d$ nonsingular. Each point of $V(x)^{\perp} / x$ arises as a first component; $V(\langle x, y\rangle)$ determines $6 \cdot 2$ second components; and $K_{x}$ acts transitively on the set of second components. Thus, the number of nonsingular points of $V(x)^{\perp} / x$ divides twelve times the number of singular points, which is not the case (since $\operatorname{rad} V=0$ ).

(VII) In (f), $V(x) / x$ is a nonsingular 2-space. There are thus 4 types of lines on $x$ : those in $V(x)$, those in $V(x)^{\perp}$, those perpendicular to exactly one line of $V(x)$ through $x$, and all others. (If $M$ is one of the latter lines, then $\left(M^{\perp} \cap V(x)\right) / x$ is nonsingular.) Thus, $K$ has more than 3 orbits of $(1,2)$-flags.

(VIII) In (g), $C_{x}$ acts irreducibly on $\left(x^{\perp} \cap W\right) / x$ : it induces at least $\Omega^{-}(4,3)$ there. We claim that $K_{x}$ acts irreducibly on $x^{\perp} / x$. For, let $M$ be a proper $K_{x}$-invariant subspace of $x^{\perp} / x$ of least dimension. If $\operatorname{dim} M \leqslant 4$, then $R$ centralizes $M$, so that $M=\left(x^{\perp} \cap W\right) / x$ and $K_{x}$ has at least 4 point-orbits on $x^{\perp} / x$ (namely, those in $M$, those in $M^{\perp}$, those on lines meeting $M$ and $M^{\perp}$, and those on no such line). Thus, $\operatorname{dim} M>4$. Also, $\operatorname{dim} M \leqslant \frac{1}{2} \operatorname{dim}\left(x^{\perp} / x\right)=m-1$ (by minimality). Since $R$ acts on $M$, we cannot have $s=3^{m-3}+1$. Thus, $s=3^{m-3}-1$. Using $C_{x}$ again, we see that $\operatorname{dim} M \neq m-2, m-1$. Consequently, $M$ is a totally singular $m-3$-space. The lines on $x$ must fall into $K_{x}$-orbits of lengths $\frac{1}{2}\left(3^{m-3}-1\right)$, $10 \cdot 3^{m-3}$ and $\frac{1}{2} 3^{m}\left(3^{m-3}-1\right)$. Let $L^{\prime}$ be any line such that $K_{L^{\prime}}^{L^{\prime}}$ is intransitive. Counting suitable $(1,2)$-flags in four ways, we find that $\left|L^{\prime K}\right| e_{i}=\left|x^{K}\right| l_{i}$ with $l_{1}$ and $l_{2}$ two of the above numbers and $e_{1}, e_{2}$ integers $\leqslant 3$. However, the equation $e_{1} l_{2}=e_{2} l_{1}$ is easily seen to have no solution.

Thus, $K_{x}$ acts irreducibly. Set $E=C_{K}(L) \cap C_{K}\left(L^{\perp} / L\right)$, so that $|E \cap C|=9$. If $e \in E$ then $\operatorname{dim}\left[x^{\perp} / x, e\right] \leqslant 2$ by $(2.1)$.

We next show that $K_{x}$ acts primitively on $x^{\perp} / x$. For, suppose that $K_{x}$ preserves a nontrivial decomposition $x^{\perp} / x=W_{1} \oplus \cdots \oplus W_{k}$. The above property of $E \cap C$ shows that $E \cap C$ cannot act faithfully on $\left\{W_{1}, \ldots, W_{k}\right\}$. Since $\left(C_{x}\right)^{\prime} \cong A_{6}$, we may assume that $\left(x^{\perp} \cap W\right) / x \subseteq W_{1}$. Then $R$ fixes $W_{1}$. Since $r \mid 3^{m-2} \pm 1$, it follows that $x^{\perp} \cap W / x=W_{1}$. But $K_{x}$ has at most 3 point-orbits on $x^{\perp} / x$. Thus, $k=2$, and $V$ has type $\Omega^{+}(10,3)$. Now the 3 point-orbits have lengths $2 \cdot 10,10 \cdot 10 \cdot 2$ and $30 \cdot 15 \cdot 2$. This leads to an impossible equation $e_{1} l_{2}=e_{2} l_{1}$ as before.

Now $K_{x}$ is primitive, $|E| \geqslant 9$, and $E$ acts faithfully on $x^{\perp} / x$ (since $A(x)=1$ by (IV)). By $(2.5), K_{x}$ induces at least $\Omega\left(x^{\perp} / x\right)$ or $2 \cdot \Omega(7,3)$ on $x^{\perp} / x$. The first case is eliminated by (2.6) or (2.14). By $(\alpha)$ in (2D), the second case leads to a subgroup $2 \cdot \Omega(7,3)$ of $K_{x}$ containing long root elements. Thus, (g) cannot occur. 
(IX) In (h), $\left|O_{2}(C)\right| \geqslant 2^{4}$. There is an involution $j \in O_{2}(C)$ inverting $L$ such that $F=[V, j]$ has type $\Omega^{+}(4,3)$. Then $j$ centralizes $L^{\perp} / L$. Since

$$
K_{L} \unrhd\langle j\rangle\left(C_{K}(L) \cap C_{K}\left(L^{\perp} / L\right)\right),
$$

by the Frattini argument we have $K_{L}^{L^{\perp}}{ }^{\perp} / L=H_{I}^{L^{\perp} / L}$, where $H=C_{K}(j)_{L}$. Note that $L^{\perp} / L$ and $F^{\perp}$ are $H$-isomorphic.

If $L^{\prime}$ is any line on $x$ such that $L^{\prime} \cap F^{\perp} \neq 0$, then $j$ acts nontrivially on $L^{\prime}$. However, if $L^{\prime \prime}$ is any line of $W$ then $N_{L^{\prime \prime}}^{L^{\prime \prime}}$ acts regularly on points since $N^{W}$ is monomial; moreover $N_{L^{\prime \prime}}^{I^{\prime \prime}}=K_{L^{\prime \prime}}^{L^{\prime \prime}}$ by the Frattini argument. Then $L^{\prime} \notin L^{K}$, and hence $L^{\prime \prime} \in L^{K}$ since $K$ has at most 2 line-orbits. In particular, $N^{W}$ is line-transitive.

Note that $K_{L^{\prime}}^{L^{\prime}} / L^{\prime}$ cannot be point-transitive. (For, if it were, then we would have $r|| K_{L^{\prime}} \mid$, and then $L^{\prime}$ would belong to $L^{K}$ by Sylow's theorem.) Thus, by the parenthetical hypotheses of (6.2), $H^{F^{\perp}}$ has at most 2 point-orbits. There are two subcases to consider: $(\alpha) H$ fixes $W$, and $(\beta) H$ moves $W$.

$(\alpha)$ Here, $H$ is transitive on the $\left(3^{m-2}+1\right)\left(3^{m-3}-1\right) / 2$ points of $F^{\perp}$. Suppose that $m \geqslant 6$. Let $r_{1}$ be a primitive divisor of $3^{m-3}-1$ (cf. (2.18)), let $R_{1} \in S y l_{r_{1}} H$, and set $Z=C_{V}\left(R_{1}\right), N_{1}=N_{K}\left(R_{1}\right)$ and $C_{1}=C_{N_{1}}\left(Z^{\perp}\right)$. Then $N_{1}^{Z}$ is point-transitive, $W \subset Z$, and $N_{1 W}^{W}=K_{W}^{W}=N^{W}$ by the Frattini argument. Moreover, $\operatorname{dim} Z=7$. Set $N_{1}^{*}=\left(N_{1}^{Z}\right)^{x^{\perp} / x}$. Then 5||$N_{1}^{*} \mid$. Also, $N_{1}^{*}$ contains an elementary abelian group of order 8 none of whose involutions induces -1 on $Z \cap x^{\perp} / x$. (Namely, $\left(N_{1 W}\right)^{Z}$ has a subgroup $Z_{2}^{3}$ centralizing $W^{\perp} \cap Z$ and fixing a member of the distinguished orthogonal basis of $W$.) Since $Z$ contains a line $L^{\prime}$ meeting both $F$ and $F^{\perp} \cap Z, N_{1}^{*}$ has 2 or 3 point-orbits, and it follows from (5.6) that $N_{1}^{*} \geqslant Z_{2}^{4} \rtimes$ $Z_{5}$. The latter group is line-transitive, so $N_{1}^{Z}$ is $(1,3)$-transitive. But then $N_{1}^{Z}$ is also $(2,3)$-transitive, whereas it is not even line-transitive (since $L, L^{\prime} \subset Z$ ). Thus, $m \leqslant 5$.

If $m=5$, then $H^{F^{\perp}}$ is a point-transitive subgroup of $\Gamma O^{-}(6,3)$. By (5.12), $C_{K}(F) \unrhd \Omega^{-}(6,3)$ or $2 \cdot \operatorname{PSL}(3,4)$. In particular, 5||$C_{K}(L) \mid$. Setting $r_{1}=5$ and proceeding as before, we again obtain a contradiction.

If $m=4$ then $H^{W^{\perp}}$ is transitive on the points of $W^{\perp}$, and on those of $F^{\perp}$ not in $W^{\perp}$. Thus, $C_{K}(F) \unrhd \Omega^{-}(4,3)$ or $A_{5}$. By (IV), $|A(x)|=3$ and $C_{K}(F) \unrhd A_{5}$. Since $A(x)<C_{K}(R)$, we have $C \unrhd Z_{2}^{4} \rtimes A_{5}$. If $y$ is a point of $W^{\perp}$, then $C$ acts on $y^{\perp} / y$. Also, $V(x)^{\perp} / x$ has type $\Omega^{+}(6,3)$. (For, $V(x)^{\perp} \cap W / x$ and $W^{\perp}$ have respective types $\Omega^{-}(2,3)$ and $\Omega^{-}(4,3)$.) Thus, $K_{y}$ induces a subgroup of Aut $P \Gamma L(4,3)$ having at most 2 line-orbits and containing the point-transitive group induced by $C$. By (5.12), $K_{y}^{y^{\perp} / y} \unrhd \Omega^{+}(6,3), \Omega(5,3)$ or $Z_{2}^{4} \rtimes A_{5}$. The latter 2 cases require that $K_{y}^{y^{\perp} / y}$ fixes more than one nonsingular point, and hence that it has more than 3 point-orbits. In the first case, $(2.14)$ produces long root groups.

( $\beta$ ) Let $h \in H$ with $W^{h} \neq W$. Then $F \subset W^{h}$. Set $Z=\left\langle W, W^{h}\right\rangle$ and $D=\left\langle C, C^{h}\right\rangle$. Then $\operatorname{dim} Z=6, D$ acts on $Z$, and $D$ centralizes $Z^{\perp}$.

Since $C_{W}(j)$ and $C_{W}(j)^{h}$ are isometric, $C_{Z}(j)$ is either anisotropic or has a nontrivial radical.

Assume that $z=\operatorname{rad} Z \neq 0$. Since $C$ and $C^{h}$ agree on $Z / z$, while centralizing $Z^{\perp}$, they are conjugate under $O_{3}(D)$. Thus $\left|O_{3}(D)\right| \neq 1$. If $1 \neq d \in O_{3}(D)$, then $\operatorname{dim} C_{Z}(d)=5$; since $d$ centralizes $Z^{\perp}$, we have $d \in A(z)$. Now $C \unrhd Z_{2}^{4} \rtimes A_{5}$ once again. Thus, $|A(z)|>3$ (since $C$ acts on $A(z)$ ), which contradicts (IV). 
Consequently, $C_{Z}(j)$ is an anisotropic 2-space. Since $C_{W}(j)$ and $C_{W}(j)^{h}$ are isometric, it follows that $Z=F \perp C_{W}(j) \perp C_{W}(j)^{h}$. However, $O_{2}(C)^{F}=O_{2}\left(C^{h}\right)^{F}$. Thus, $\mathrm{O}_{2}(C)$ and $\mathrm{O}_{2}\left(C^{h}\right)$ commute.

Now $N=\left\langle\mathrm{O}_{2}(\mathrm{C})^{H}\right\rangle$ is an elementary abelian normal subgroup of $H$, and acts nontrivially on $F^{\perp}$. Then $H$ acts monomially on $\left[F^{\perp}, N\right]$, while having at most 2 point-orbits on $F^{\perp}$. Thus, $2 m-3=5$ and $H^{F^{\perp}} \geqslant Z_{2}^{4} \rtimes Z_{5}$. Then $C_{K}(F)^{F^{\perp}} \geqslant Z_{2}^{4} \rtimes Z_{5}$.

Let $\left\{e_{i}\right\}$ be an orthogonal basis of $\left\langle F^{\perp}, x\right\rangle / x$ with respect to which $C_{K}(F)$ is monomial. We may assume that $\left(e_{i}, e_{i}\right)=1$. Let $\{e, f\}$ be a basis of $x^{\perp} \cap F / x$ with $(e, e)=1=-(f, f)$. We may assume that $R^{F^{\perp}}$ is generated by $g=(1,2,3,4,5)$. Then $C_{\bar{V}}(g)=\left\langle e, f, \Sigma_{i} e_{i}\right\rangle$, where $\bar{V}=x^{\perp} / x$. Consequently, $N_{x}$ acts transitively on $\left\{\langle e+f\rangle,\langle e-f\rangle,\left\langle e+\sum_{i} e_{i}\right\rangle,\left\langle e-\sum_{i} e_{i}\right\rangle\right\}$. Set $K^{*}=K_{x}^{x^{1} / x}$ and $\Omega=\langle e+f\rangle^{K^{*}}$. Then $C_{K}(F)$ acts on $\Omega$, and hence $\Omega$ contains all the points $\left\langle e+\sum_{i} \varepsilon_{i} e_{i}\right\rangle$ with each $\varepsilon_{i}= \pm 1$. Then $\Omega$ contains two perpendicular points, such as $\left\langle e+\sum_{i} e_{i}\right\rangle$ and $\left\langle e+e_{1}+e_{2}-e_{3}-e_{4}-e_{5}\right\rangle$. Also, $C_{K}(F)$ is transitive on the lines of $\bar{V}$ through $\langle e+f\rangle$. By (2.10), $K^{*}$ fixes a hyperplane. The only one available is $f^{\perp}$, which has type $\Omega^{+}(6,3)$. This produces the same contradiction as in the last part of $(\alpha)$.

(X) In (i), $C^{W}$ has a normal extraspecial subgroup of order $2^{5}$. Let $j$ be an involution in $C$ such that $[V, j]$ is a hyperbolic line, and let $x \in[V, j]$. Then $j$ inverts $x$ and centralizes $x^{\perp} / x$.

By (IV), $Q \cap K=1$. Thus, $\langle j\rangle=C_{K}\left(x^{\perp} / x\right) \leqslant Z\left(K_{x}\right)$. Write $j_{x}=j$ and $U(x)=\left[V, j_{x}\right]$. If $y \in U(x)$ then $U(y)=U(x)$. If $y \in U(x)^{\perp}$, then $j_{x}$ centralizes $j_{y}$ and $U(x) \cap U(y)=0$, so that $U(y) \subseteq U(x)^{\perp}$.

Let $y \in U(x)^{\perp}$ and $z \in U(x)^{\perp} \cap U(y)^{\perp}$, and consider $S=\langle U(x), U(y), U(z)\rangle$. If $s \in S^{\perp}$ then $U(s) \subseteq S^{\perp}$. Thus, $S^{\perp}=\left\langle U(s) \mid s \in S^{\perp}\right\rangle$. Similarly, $S=\langle U(s) \mid s \in S\rangle$. There are $\left(3^{6}-1\right) /\left(3^{2}-1\right)=91$ hyperbolic lines $U(s)$ in $S$, of which $\left(3^{4}-1\right) /\left(3^{2}-1\right)=10$ lie in $U(s)^{\perp} \cap S$. Call each such $U(s)$ a Point, and each of these sets of 10 Points of $S$ a Line. Then two distinct Points are in at most one Line. A standard counting argument shows that we have a projective plane of order 9. Since $U(s) \rightarrow U(s)^{\perp} \cap S$ induces a polarity without absolute Points, this contradicts a result of Baer [10, p. 152].

(XI) In (j), there are two possibilities for $N^{W}$, which are dual to examples $(2.1,2)_{\mathrm{iii}}$ of $(5.13)$. In both cases, $\left|O_{3}(C)\right| \geqslant 3^{3}$. Let $\langle i\rangle$ be a subgroup of order 3 in $O_{3}(C)$ such that $\langle i\rangle^{W}$ is central in a Sylow 3-subgroup of $\Omega^{-}(6,2)$. Then $[V, i]=W$, and there are exactly 9 lines of $V$ fixed but not centralized by $i$, all of which lie in $W$.

There is also an element $j$ of $O_{3}(C)$ such that $F=[V, j]$ has type $\Omega^{+}(4,2)$ and for which there are exactly 3 lines of $V$ fixed but not centralized by $j$, all of which lie in $F$. We may assume that $x \in L \subset F$, and that $L$ is the fixed line of $j$ through $x$. Note that $\langle j\rangle$ is transitive on $L$, while $\langle i\rangle$ is transitive on the second line $L^{\prime}$ of $F$ through $x$. Also, $L^{K} \neq L^{\prime K}$ since $L^{N} \neq L^{\prime N}$.

If $E$ is any plane containing $L$, then $j$ fixes $E$ and $K_{L E}^{L}$ is transitive. If $E^{\prime}$ is any plane containing $L^{\prime}$ and fixed by $i$, then $K_{L^{\prime} E^{\prime}}^{L^{\prime}}$ is transitive. Here, $E^{\prime} \notin E^{K}$. (For otherwise, some conjugate $i^{\prime}$ of $i$ acts nontrivially on $E$. By Sylow's theorem, $\left[E, i^{\prime}\right]$ 
and $[E, j]$ are in the same line-orbit of $K_{E^{\prime}}^{E^{\prime}}$, and hence of $K$, which is not the case.) Thus, $E^{K}$ and $E^{\prime K}$ are the two plane-orbits of $K$ (cf. (6.3 i)).

In particular, all planes containing $L$ lie in $E^{K}$. Moreover, $K_{E}^{E}$ is transitive on the members of $L^{K}$ lying in $E$ (again by Sylow's theorem). By considering $(L, E)^{K}$, it follows that $K_{L}$ is point-transitive on $L^{\perp} / L$.

Since $\langle j\rangle\left(C_{K}(L) \cap C_{K}\left(L^{\perp} / L\right)\right) \unlhd K_{L}$, we have $K_{L}^{L^{\perp} / L}=H^{L^{\perp} / L}$ by the Frattini argument, where $H=N_{K}(\langle j\rangle)_{L}$. Then $H$ is point-transitive on $L^{\perp} / L$, and hence also on $F^{\perp}$. Since $\langle j\rangle$ is transitive on $L$, it follows that $H_{x}$ is also point-transitive on $F^{\perp}$. Here, $H_{x}$ fixes both $L$ and $L^{\prime}$. Consequently, $K_{L^{\prime} F}$ is transitive on $F^{\perp}$, and hence on $L^{\prime \perp} / L^{\prime}$.

Some plane contains lines from both of the orbits $L^{K}$ and $L^{\prime K}$ (cf. (2.13)). In view of the transitivities we have found, $K$ must be transitive on planes, which is not the case.

This completes the proof of (6.2).

THEOREM 6.4. Let $K$ be a subgroup of $\Gamma(V)$, where $V$ has rank $n \geqslant 3$. If $K$ is transitive on points, lines and planes, then either $K \geqslant \operatorname{Chev}(V)$ or $K$ is $A_{7}$ or $S_{7}$ inside $\mathrm{O}^{+}(6,2)$.

Proof. By $(5.15,5.17,5.21)$, we may assume that $n \geqslant 4$ and that $V$ does not have type $\Omega^{+}(8, q)$. By $(6.1)$, we may also assume that $V$ is not unitary.

By Table IV, a suitable prime divisor $r$ of the number of planes can be used in (6.2), except for cases listed in (6.3), and except when a candidate for $r$ already divides the number of lines. A straightforward check shows that we must only consider the cases $\Omega^{\star}(10, q), \Omega^{ \pm}(12, q), \operatorname{Sp}(8, q), \Omega(9, q), \Omega^{-}(14, q)$ and $\Omega^{-}(18, q)$.

For $m=5$ and 6 , the number of planes of an $\Omega^{+}(2 m, q)$ space is divisible by $\left(q^{m-3}+1\right)^{2}$. Thus, (6.2) applies, except in the case of $\Omega^{+}(12,2)$.

Similarly, in the cases $\operatorname{Sp}(8, q), \Omega(9, q)$, and $\Omega^{-}(14, q),\left(q^{2}+1\right)^{2}|| K \mid$; while for $\Omega^{-}(18, q),\left(q^{3}+1\right)^{2}|| K \mid$. Thus, (6.2) applies, except in the case $\Omega^{-}(18,2)$.

For $\Omega^{-}(12, q)$, the number of planes is divisible by $\left(q^{2}+1\right)^{2}$. Let $r$ be a primitive divisor of $q^{2}+1$, and let $R \in S y l_{r} K_{L}$. Define $W$ and $C$ as usual. Since $R \neq 1, W$ has type $\Omega^{-}(6, q)$ or $\Omega^{+}(8, q)$. The first possibility is dealt with exactly as in (6.2). If $W$ has type $\Omega^{+}(8, q)$, let $r^{*}$ be a primitive divisor of $q^{3}-1$. Then $N$ contains an element of order $r^{*}$. Since $r^{*}|| N^{W^{\perp}} \mid$, we can apply (6.2) with $r^{*}$ in place of $r$.

For $\Omega^{-}(10, q)$, the number of lines is divisible by $\left(q^{3}-1\right) /(q-1)$. Let $d$ be a primitive divisor of $q^{3}-1$. If $D \in S y l_{d} K$, then $C_{V}(D)$ has type $\Omega^{-}(4, q)$. Let $r$ be a primitive divisor of $q^{2}+1$. Since $N_{K}(D)$ is point-transitive on $C_{V}(D)$, its order is divisible by $r$. An element of order $r$ in $N_{K}(D)$ must centralize $C_{V}(D)^{\perp}=[V, D]$ (since $r|| N_{K}(D)^{[V \cdot D]} \mid$ ). Consequently, (6.2) applies.

Similarly, in the case $\Omega^{+}(12,2)$ we find that $|K|$ is divisible by $d=2^{4}+1$. If $D \in S y l_{d} K$, then $C_{V}(D)$ has type $\Omega^{-}(4,2)$, and an element of order $r=5$ in $N_{K}(D)$ centralizes $C_{V}(D)^{\perp}$. If $E$ is a plane of $C_{V}(D)^{\perp}$, then $r|| C_{K}(E) \mid$. Let $R \in S y l_{r} C_{K}(E)$, and define $W, N$ and $C$ as usual. Then $W$ has type $\Omega^{-}(8,2)$. By (2.12), $K$ is $(1,3)$-transitive. Thus, $N^{W}$ is $(1,3)$-transitive. By (5.17) and Table II, $N^{W} \geqslant \Omega^{-}(8,2)$. Then also $C^{W} \geqslant \Omega^{-}(8,2)$, and (2.4) applies. 
In the case of $\Omega^{-}(18,2)$ set $d=2^{7}-1$ and $r=2^{2}+1$. If $D \in S y l_{d} K$, then $C_{V}(D)$ has type $\Omega^{-}(4,2)$. Once again, some element of order $r$ centralizes $C_{V}(D)^{\perp}$. Let $R \in S y l_{r} K_{L}$. Then $W$ has type $\Omega^{+}(6,2), \Omega^{-}(10,2)$ or $\Omega^{+}(14,2)$, and $N^{W}$ is transitive on lines and planes (by Table IV). In the second and third cases, $N^{W}$ is also transitive on points (since $\theta_{2} \supset \theta_{1}$ by Table II); then $N^{W} \geqslant \Omega(W)$, and we can proceed as usual. Suppose that $\operatorname{dim} W=6$. Then $N^{W} \leqslant O^{+}(6,2) \cong S_{8}$. It follows that $C^{W} \geqslant A_{7}$ or $A_{8}$, and we can proceed as in steps (II) and (VI) of (6.2) in order to obtain a contradiction.

Finally, in the case of $S p(10,2), K$ is $(1,2)$-transitive by (2.12). Let $R \in \mathrm{Syl}_{3} C_{K}(L)$. Then $R$ has index $3^{2}$ in a Sylow 3-subgroup of $K$, and $R \neq 1$ since $3^{3}$ divides the number of planes. Note that $\operatorname{dim} W$ is 4,6 or 8 . If $\operatorname{dim} W>4$ then $N^{W}$ is transitive on points, lines and planes; but then $N^{W} \geqslant S p(W)$, and we obtain the usual contradiction. Thus, $\operatorname{dim} W=4$ and $N^{W}$ is flag-transitive. It follows that $C^{W} \geqslant A_{6}$, and we can again proceed as in steps (II) and (VI) of (6.2) in order to complete the proof of (6.4).

REMARK. By Tables II and III, if $n \geqslant 5$ then plane-transitivity implies both pointand line-transitivity.

THEOREM 6.5. Let $V$ have rank $n \geqslant 3$. If $K$ is a subgroup of $\Gamma(V)$ which is transitive on points, lines and $n$-spaces (of at least one type, when $V$ has type $\Omega^{+}(2 n, q)$ ), then either $K \geqslant \operatorname{Chev}(V)$ or $K$ is $A_{7}$ or $S_{7}$ inside $O^{+}(6,2)$.

Lemma 6.5'. Let $V$ have rank $n \geqslant 4$, but not have type $\Omega^{+}(8, q)$. Then $\Gamma(V)$ has no subgroup $K$ having the following properties:

(i) $K$ is transitive on points and $n$-spaces (of each type, when $V$ has type $\Omega^{+}(2 n, q)$ );

(ii) $K$ has 2 orbits of lines, at most 3 orbits of (1,2)-flags, and at most 2 orbits of planes and $(1, n)$-flags ( for each type on $n$-space, when V has type $\Omega^{+}(2 n, q)$ ); and

(iii) if $K$ has more than 3 orbits of $(2,3)$-flags, then it has 4 such orbits, as well as 2 orbits of planes and $(1, n)$-flags (of each type); if, in addition, $n=4$, then there are 7 orbits of (1,2,3)-flags and 9 orbits of flags.

Proof OF (6.5) AND (6.5'). The proof parallels that of (6.4). By $(5.15,5.17,5.21)$, we may assume that $n \geqslant 4$ and that $V$ does not have type $\Omega^{+}(8, q)$. By $(2.18)$ and Table IV, we can use the number of $n$-spaces in order to find a prime $r$ required in (6.2), except for cases occurring in (6.3), or cases in which a candidate for $r$ already divides the number of lines. Thus, this time we must consider the cases $\Omega^{ \pm}(10, q)$, $\Omega^{+}(12, q), S U(8, q), S U(10, q), S p(10,2), \Omega^{-}(12,2), S p(8, q)$ and $\Omega(9, q)$.

For $\Omega^{+}(10, q), q \neq 2$, use primitive divisors $d$ of $q^{3}+1$ and $r$ of $q^{2}+1$. If $D \in S y l_{d} K$ then $C_{V}(D)$ has type $\Omega^{-}(4, q)$, and hence $N_{K}(D)$ has an element of order $r$. That element cannot act nontrivially on $C_{V}(D)^{\perp}$. Thus, (6.2) applies.

The case $S U(8, q), q \neq 2$, is handled in the same manner, using primitive divisors $d$ of $q^{5}+1$ and $r$ of $q^{3}+1$. Also, for $S U(10, q)$ we find that $\left(q^{3}+1\right)^{2}$ divides $|K|$ (Table IV), so that (6.2) applies when $q \neq 2$.

For $S U(2 n, 2), n=4$ or 5 , let $M$ be an $n$-space. By (2.12), $K_{M}^{M}$ is point-transitive if $n=4$, and has order divisible by $2^{5}-1$ if $n=5$. By hypothesis, $K_{M}^{M}$ has at most 2 
point-orbits. Now $(5.1,5.2)$ imply that $K_{M}^{M}$ is transitive unless $n=5$ and $K_{M}^{M} \geqslant S L(5,2)$. If $n=5$ and 7||$K \mid$, then (6.2) applies. Thus, we may assume that $K$ is $(1, n)$-transitive. By $(6.1)$ and $\left(6.5^{\prime} \mathrm{ii}\right.$, iii), there is a line $L$ such that $K_{L}$ is point-transitive on $L^{\perp} / L$. If $n=4$, then $2^{2}+1|| C_{K}(L) \mid$ by $(5.12)$; if $n=5$ then $2^{5}+1|| K_{L} \mid$. In either case, (6.2) yields a contradiction.

For $\Omega^{-}(10, q)$, assume first that $K$ is line-transitive. Then primitive divisors of $q^{3}-1$ and $q^{2}+1$ can be used as before. Now assume that we are in the situation of $\left(6.5^{\prime}\right)$. Then $K$ has 2 orbits of $(1,4)$-flags, and hence also 2 of $(3,4)$-flags and at most 2 of planes. If $K$ is plane-transitive then it is also line-transitive (by Table II), and (6.4) applies. If $K$ has 2 plane-orbits, then $K_{E}^{E^{\perp} / E}$ is point-transitive for each plane $E$; thus, $q^{2}+1$ divides $\left|K_{E}\right|$, and (6.2) applies.

In the cases $\Omega^{+}(10,2), \Omega^{-}(12,2)$ and $S p(10,2), K$ is $(1,5)$-transitive (by Table IV and (2.12)). Let $M$ be a 5-space. If $\left|K_{M}^{M}\right|$ is even, then $K_{M}^{M}=S L(5,2)$ by $(5.5)$. Thus, $\left|K_{M}^{M}\right|$ is odd. However, $|K|$ is even. (A Sylow 31-subgroup $S$ fixes exactly two 5-spaces, which $N_{K}(S)$ permutes transitively.) Let $t$ be an involution in $K$. Then we may assume that $t$ fixes $M$, and hence centralizes $M$. But now it is easy to find another fixed 5-space of $t$ not in $C_{V}(t)$.

For $\Omega^{+}(12, q)$, let $d$ and $r$ be primitive divisors of $q^{4}+1$ and $q^{2}+1$, respectively. Using a Sylow $d$-subgroup of $K$, we find (as earlier in the present proof) an element of order $r$ centralizing a subspace $W$ of type $\Omega^{-}(8, q)$. Let $R \in S y l_{r} C_{K}(W)$. If $R \in S y l_{r} K$, then $N^{W}$ is transitive on the $\left(q^{4}+1\right)\left(q^{3}-1\right) /(q-1)$ points of $W$, and (6.2) applies with $s=q^{3}-1$. Thus, we may assume that $R$ is not Sylow in $K$ (and that $N^{W}$ is not point-transitive).

If $K$ is line-transitive, then $r|| N_{L}^{W} \mid$ for every line $L$ of $W$. Then also $r|| C_{N}(L)^{W} \mid$. Fix $x \in W$, and set $N^{*}=N^{x^{\perp} \cap W / x}$. Each point of $\left(x^{\perp} \cap W\right) / x$ is fixed by an element of $N^{*}$ of order $r$. By $(5.10), N^{*(\infty)}=\Omega^{-}(6, q)$ or $2 \cdot \operatorname{PSL}(3,4)$. In the former case, (2.14) produces long root elements in $K$; while in the latter case, (6.2) applies with $s=q^{3}+1$.

Thus, we must now consider $\left(6.5^{\prime}\right)$ for $\Omega^{+}(12, q)$. Here, $N^{W}$ has at least 2 point-orbits and at most 4 orbits of $(1,3)$-flags. As in the preceding paragraph, we find that there are 2 point-orbits and 4 orbits of $(1,3)$-flags, both for $N^{W}$ and for $K$. In particular, each plane-orbit of $K$ has a member in $W$. Since $q^{2}+q+1$ divides the number of points of $V$ but not the number of planes, there is a plane $E$ such that $\left|K_{E}^{E}\right|$ is divisible by a primitive divisor $r^{*}$ of $q^{2}+q+1$. We may assume that $E \subset W$. By the Frattini argument, $K_{E}^{E}=N_{E}^{E}$. Thus, $r^{*}|| N_{E}^{W} \mid$. An element of order $r^{*}$ in $N_{E}$ centralizes $W^{\perp}$, and hence also centralizes a line of $V$. Once again, (6.2) applies with $s=q^{3}-1$, and finishes the case $\Omega^{+}(12, q)$.

The cases $\operatorname{Sp}(8, q)$ and $\Omega(9, q)$ are somewhat different from the preceding ones, in that a suitable prime is harder to find. Suppose first that we are in $\left(6.5^{\prime}\right)$. If $L$ is a line, we may assume that $q^{2}+1|| K_{L} \mid$, as otherwise (6.2) applies. In particular, $K_{L}^{L^{\perp} / L}$ is intransitive on both points and lines. Thus, we must be in $\left(6.5^{\prime}\right.$ iii). Let $\left(x_{i}, L_{i}\right), i=1,2,3$, be representatives of the orbits of $(1,2)$-flags; let $K_{i}$ be the corresponding group induced on $L_{i}^{\perp} / L_{i}$. Since $K$ has 7 orbits of $(1,2,3)$-flags, we may assume that $K_{1}$ and $K_{2}$ have 2 point-orbits, while $K_{3}$ has 3 point-orbits. Since 
$K_{1}$ has at least 2 line-orbits, it has at least 3 flag-orbits; and so does $K_{2}$. Thus, $K_{3}$ has at most 3 flag-orbits. Then $\left(K_{3}\right)_{y}$ is transitive on $y^{\perp} / y$ for each point $y$ of $L_{3}^{\perp} / L_{3}$. Consequently, $K_{3}$ is line-transitive, which is not the case.

Thus, we must be in the situation of (6.5): $K$ is transitive on the $\left(q^{4}+1\right)\left(q^{3}+1\right)\left(q^{2}+1\right)\left(q^{2}+q+1\right)$ lines of $V$. By $(2.12)$, if $F$ is a 4-space then $q^{2}+q+1$ divides the length of each line-orbit of $K_{F}^{F}$. Since $K$ is not flag-transitive, $K_{F}^{F}$ fixes a point or a plane (by (5.1)). In either case, there is a plane $E \subset F$ such that $K_{E F}^{E}$ is transitive. By Sylow's theorem, $K_{F}^{F}$ is transitive on the set of such planes. Hence, $K_{E}^{E^{\perp} / E}$ is also transitive.

Consequently, there is an orbit of $\left|L^{K}\right| \alpha(q+1)=\left|F^{K}\right| \varepsilon\left(q^{2}+q+1\right)$ triples ( $L, E, F$ ), where $\alpha$ and $\varepsilon$ are the numbers of planes $E$ containing $L$ and contained in $F$, respectively. Here, $\varepsilon$ is 1 or $q^{3}$ (cf. (2.7 i) and (2.14)), and hence so is $\alpha$ (by Table IV). Then $\alpha=1$. (For, if $\alpha=q^{3}$, a Sylow $p$-subgroup of $K_{L}$ acts transitively on the corresponding $q^{3}$ points of $L^{\perp} / L$. Then these are the points not in some line of $L^{\perp} / L$. Thus, $K_{L}$ fixes a 4 -space containing $L$, and this contradicts (2.12).)

Note that $K_{E}^{E} \neq S L(3, q)$ if $q>2$. For, suppose that $K_{E}^{E} \geqslant S L(3, q)$ and $q \neq 2$. Then $C_{K}\left(E^{\perp} / E\right)^{E} \geqslant S L(3, q)$. By $(2.14), K_{E}$ has a subgroup $S \cong S L(3, q)$ fixing two planes. Then $S_{x}$ has an element of order $p$ centralizing $x^{\perp} / x$. This is impossible by (2.1) and (2.4).

Now suppose that $q \neq 2,8$. Recall that $K_{L}=K_{L E}$, and that $K_{L E}^{E^{\perp} / E}$ is transitive. There is a prime $r \neq 3$ dividing $q+1$ but not dividing $\log _{p} q$. Let $R \in S y l_{r} K_{L}$. Then $R^{E}=1$ by the preceding paragraph and $(2.7 \mathrm{i})$, so that $\operatorname{dim} W=6$ or $7, N^{W}$ is a line-transitive subgroup of $\Gamma \operatorname{Sp}(6, q), \Gamma O^{+}(6, q)$ or $\Gamma O(7, q), q^{2}+1|| N^{w} \mid$, and hence (6.2) applies.

Thus, $q$ is 2 or 8 . Let $d$ be a primitive divisor of $q^{3}-1$, and let $D \in S y l_{d} K_{E}$. Then $N_{K}(D)$ is transitive on the $2(q+1)$ fixed 4-spaces of $D$. Thus, $|K|$ is even, and hence so is $\left|K_{L}^{L}\right|=\left|K_{L E}^{L}\right|$. Then $K_{E}^{E} \geqslant S L(3, q)$. A Sylow 3-subgroup of $K_{E F}$ centralizes a line. If $R \in \mathrm{Syl}_{3} C_{K}(L)$, we obtain the same contradiction as before.

This completes the proof of $(6.5)$ and $\left(6.5^{\prime}\right)$.

The remainder of this section degenerates into those types of technical results which dominated $\S 5$ (and resemble $\left(6.5^{\prime}\right)$ ). They appear here only because they will be needed in (8.3): there, $(6.6)$ and (6.8) are used to deal with $(n-2 \mid 2)$, while (6.7) and (6.9) are used for $(n-3 \mid 3)$.

Lemma 6.6. Suppose that $n \geqslant 4$ but $V$ does not have type $\Omega^{+}(8, q)$. Then $\Gamma(V)$ has no subgroup $K$ with the following properties:

(i) $K$ is point-transitive;

(ii) $K$ has 2 orbits of lines, planes and $(1,2)$-flags;

(iii) $K$ has 3 orbits of (1,3)- and (2,3)-flags; and

(iv) if $n=8$ then $K$ has 2 plane-orbits and 3 orbits of $(3,4)$-flags.

Proof. By (ii) and (iii), there is a line $L$ such that $K_{L}$ is transitive on $L^{\perp} / L$. By Table IV and (2.18), $\left|K_{L}\right|$ is divisible by a prime required in (6.2), unless $V$ has type $S p(10,2), \Omega^{+}(12,2)$ or $\Omega^{-}(18,2)$ (cf. $\left.(6.3)\right)$. Thus, by $(6.2)$ we only need to consider these three cases. 
If $V$ has type $S p(10,2)$, then 7||$K_{L}^{L^{\perp} / L} \mid$, and the proof of (6.2) can be repeated in order to obtain a contradiction.

If $V$ has type $\Omega^{+}(12,2)$, set $d=2^{4}-1$ and $r=3$. Since $d|| K_{L} \mid$, a Sylow $d$-subgroup of $K$ centralizes a subspace $W_{0}$ of type $\Omega^{+}(4,2)$. There is then a group of order 9 centralizing $W_{0}^{\perp}$. Let $E$ be a plane of $W_{0}^{\perp}$; if possible, choose $E$ so that $K_{E}^{E}$ is transitive (such planes exist in $V$, by (ii) and (iii)). Let $R \in S y l_{3} C_{K}(E)$. Then $W=C_{V}(R)=W_{0}^{\perp}$. If 7 divides $\left|N^{W}\right|$, then an element of order 7 in $N$ centralizes $W^{\perp}$, and (6.2) applies. Since $K_{E}^{E}=N_{E}^{E}$ by the Frattini argument, $K_{E}^{E}$ cannot be transitive. Thus (by (ii)), all planes of $W$ lie in the same $W$-orbit. Consequently, $N^{W}$ is transitive on planes; but then 7 again divides $\left|N^{W}\right|$.

If $V$ has type $\Omega^{-}(18,2)$, then (iv) provides us with a plane $E$ such that $K_{E}$ is point-transitive on $E^{\perp} / E$. Then $2^{6}+1|| K_{E} \mid$, and (6.2) yields a contradiction.

Lemma 6.7. Suppose that $n \geqslant 4$ but $V$ does not have type $\Omega^{+}(8, q)$. Then $\Gamma(V)$ has no subgroup $K$ with the following properties:

(i) $K$ is (1,2)-transitive;

(ii) $K$ has 2 orbits of planes, (1,2,3)-flags and 4-spaces; and

(iii) $K$ has 3 orbits of $(1,4)$ - and $(3,4)$-flags.

Proof. By (6.1), $V$ is not unitary. Set $K^{*}=K_{x}^{x^{\perp} / x}$. Then $K^{*}$ is transitive on points and has 2 orbits of lines and of $(1,2)$-flags. Clearly, $(6.2)$ applies to neither $K$ nor $K^{*}$. However, by (ii) and (iii) there is a plane $E$ such that $K_{E}$ is point-transitive on $E^{\perp} / E$. By Table IV and (2.18), the failure of (6.2) implies that $V$ has type $S p(12,2), \quad \Omega^{-}(12,2), \quad \Omega^{+}(14,2), S p(8, q), \quad \Omega(9, q), \Omega^{+}(10, q), \quad \Omega(2 n+1,3)$ or $\Omega^{-}(2 n+2,2)$.

If $V$ has type $\Omega^{+}(14,2)$, then $r=2^{2}+1|| K_{E} \mid$. Let $R \in S y l_{r} K_{L}$. Then $N^{W}$ is a $(1,2)$-transitive subgroup of $\mathrm{\Gamma O}^{-}(10,2), \mathrm{\Gamma O}^{+}(8,2)$ or $\mathrm{\Gamma O}^{+}(6,2)$. In the first case, $2^{4}+1$ divides the number of lines of $W$, and (6.2) applies to $K$. In the remaining cases, $N^{W}$ contains $\Omega^{+}(W)$ or $A_{7}$ (by (5.15), (5.21)), and we can proceed exactly as in steps (II) and (VI) of the proof of (6.2).

If $V$ has type $S p(12,2)$, a Sylow 3-subgroup of $K_{E}$ has all its point-orbits on $E^{\perp} / E$ of length at least 9. Thus, there is a 3-group centralizing $E$ but fixing no point of $E^{\perp} / E$. Let $R \in S y l_{3} K_{x L}$ for $x \in L$. Then $N^{W}$ is a $(1,2)$-transitive subgroup of $S p(4,2)$ or $S p(6,2)$. Either $N^{W}$ or $\left(N_{x}\right)^{\left(x^{\perp} \cap W\right) / x}$ contains $S p(2,4)$, and we can again proceed exactly as in (6.2).

If $V$ has type $\Omega(2 n+1,3), n \geqslant 5$, let $r$ be a primitive divisor of $3^{n-3}+1$, and note that $r|| C_{K}(E) \mid$. Let $R \in S y l_{r} C_{K}(L)$. Then $N^{W}$ is a $(1,2)$-transitive subgroup of $\Gamma O(7,3)$. By $(6.3 \mathrm{i})$, it is even flag-transitive, and we obtain a contradiction from (2.6) just as in step (II) of (6.2).

If $V$ has type $\Omega^{-}(2 n+2,2), n \neq 5$, let $r$ be a primitive divisor of $2^{n-2}+1$, and let $R \in \operatorname{Syl}_{r} C_{K}(L)$. Then $N^{W}$ is a $(1,2)$-transitive subgroup of $\Gamma O^{-}(8,2)$. By $(6.3 \mathrm{i})$ and (2.1), $\left|O_{3}\left(N_{x}^{W}\right)\right| \geqslant 27$. Thus, $N_{x}^{W}$ fixes a second point $y$, and is point-transitive on $\langle x, y\rangle^{\perp}$. Then $y$ is uniquely determined, and $\{x, y\}$ is an imprimitivity block for the action of $N$ on the points of $W$. Since $W$ has an odd number of points, this is impossible. 
Consider the remaining cases. If $E$ is any plane then $K_{E}^{E}$ is flag-transitive. Thus, by (2.7 i), $C_{K}\left(E^{\perp} / E\right)^{E}$ is transitive on $E$. Let $F$ be any 4-space. If $E \subset F$ then $K_{E F}^{E}$ is transitive. Thus, $K_{F}^{F}$ is transitive. This contradicts (ii) and (iii).

LemMa 6.8. $\mathrm{\Gamma O}^{+}(10, q)$ has no subgroup transitive on points and having 2 orbits of each type of 5-spaces, 3 orbits of planes and of each type of $(1,5)$-flags, and 4 orbits of $(1,3)$-flags.

Proof. Let $K$ be such a subgroup. Let $r$ be a primitive divisor of $q^{3}-1$. There are two plane-orbits $E_{i}^{K}, i=1,2$, such that $K_{E_{i}}$ is transitive on $E_{i}$. In particular, $r|| K_{E_{i}} \mid$. Let $R \in S y l_{r} K$; note that a Sylow $r$-subgroup of $\mathrm{\Gamma O}^{+}(10, q)$ is cyclic, and fixes exactly 2 planes.

We may assume that $R$ fixes $E_{1}$ and $E_{2}$. Clearly, $R$ centralizes $E_{i}^{\perp} / E_{i}$. Let $M$ be a 5 -space containing $E_{1}$. Since $R$ fixes only one plane of $M$, Sylow's theorem shows that $M$ contains no member of $E_{2}^{K}$. But there are only 2 orbits of 5 -spaces having the same type as $M$. Thus, we can choose $E_{1}$ so that $K_{M}^{M}$ is transitive (recall that there are 3 orbits of each type of (1,5)-flags). Now (5.5) implies that $K_{M}^{M} \geqslant S L(5, q)$.

Set $Q=O_{p}\left(\Gamma O^{+}(V)_{M}\right)$. Then $Q$ is elementary abelian of order $q^{10}$, and $K_{M}^{M}$ acts on $Q$ as it does on skew-symmetric 2-tensors. Hence $K_{M}$ acts irreducibly. Since $Q$ contains long root groups, we have $Q \cap K=1$ by (2.4). Since $H^{1}(S L(5, q), Q)=0$ (Jones and Parshall [30]), $K_{M}$ must fix a second 5-space. But then $K_{M}$ contains long root groups, which is not the case.

Lemma 6.9. If $K$ is a $(1,2)$-transitive subgroup of $\Gamma O^{+}(14, q)$, then $K \geqslant \Omega^{+}(14, q)$.

Proof. Let $d$ and $r$ be primitive divisors of $q^{5}+1$ and $q^{2}+1$, respectively. Note that $d$ divides the number of lines. If $D \in S y l_{d} K$, then $C_{V}(D)$ has type $\Omega^{-}(4, q)$. By Sylow's theorem, $r|| N_{K}(D) \mid$. An element of order $r$ in $N_{K}(D)$ cannot act nontrivially on $[V, D]$. Thus, $r|| K_{L} \mid$ for some line $L$.

Let $R \in S y l_{r} K_{L}$, and define $W$ and $N$ as usual. Then $W$ has type $\Omega^{+}(6, q)$ or $\Omega^{-}(10, q)$, and $N^{W}$ is $(1,2)$-transitive. The first possibility for $N^{W}$ is handled as in (6.2) (cf. (5.15)). If $W$ has type $\Omega^{-}(10, q)$ then $\left(q^{3}-1\right) /(q-1)$ divides the number of lines of $W$, so that (6.2) applies with $s=q^{3}-1$.

Remark. A similar argument deals with $(1,2)$-transitive subgroups of $\Omega *(2 m, q)$ whenever $m$ is relatively small.

7. Theorem 1.1: Reductions. Let $G$ and $K$ be as in (1.1), but assume that the pair $G, K$ is not listed in that theorem. We will assume, until $\S 9$, that $G$ actually acts on the vector space $V$ as a group of semilinear maps. We may also assume that $\operatorname{rad} V=0$. The case of $V$ of type $\Omega^{+}(6, q)$ will be postponed until $\S 10$.

Note that $K$ is a maximal subgroup of $G$.

Write $\varphi=1_{K}^{G}=1+\chi+\zeta$ with $\chi$ and $\zeta$ irreducible characters.

Let $B$ be a Borel subgroup of $G$. To each irreducible character $(\alpha \mid \beta)$ or $(\alpha \mid \alpha)^{+}$ of the Weyl group of $G$ there naturally corresponds an irreducible constituent of $1_{B}^{G}$, also called $(\alpha \mid \beta)$ or $(\alpha \mid \alpha)^{ \pm}$, as explained in $\S 4$. 
Throughout $\S \S 7-9$, frequent reference will be made to Tables II and III of $\S 3$. Recall from $\S 4$ the fact that, for example, the permutation character $1\left(1^{2} ; n-2\right)$ appearing in Table II is just the permutation character $\theta_{12}$ of $G$ on the set of $(1,2)$-flags of $V$. The proof of (1.1) will involve computing inner products of $\varphi$ with several such permutation characters. Some such characters not explicitly listed in a table are implicitly in the table, because of (4.4); for example, $\theta_{23}=\theta_{13}$. Most relevant inner products for $D_{n}$ are in Table II, not Table III, because of $(3.5,6,7)$; consequently, we will be able to deal with $\Omega^{+}(2 n, q)$ and $O^{+}(2 n, q)$ simultaneously in most situations.

LEMMA 7.1. Every noncentral normal subgroup of $K$ is irreducible on $V$.

Proof. Let $N$ be a noncentral reducible normal subgroup, and let $W$ be a minimal proper $N$-subspace. Then $\operatorname{rad} W=0$ or $W$. If $N=K$, it is straightforward to check whether $G$ has rank 3 on $W^{G}$; only instances on our list can occur (namely, (1.1 i, ii, iii, iv) arise in the present context). Thus, $K$ is irreducible and (by Clifford's theorem) $V=W_{1} \oplus \cdots \oplus W_{r}$ with $W_{1}=W$ and each $W_{i}$ an $N$-subspace conjugate to $W_{1}$ under $K$. The maximality of $K$ forces $K$ to be the stabilizer of the set $\left\{W_{1}, \ldots, W_{r}\right\}$. Once again it is easy to check whether $G$ has rank 3 on $W^{G}$.

Lemma 7.2. If $n \geqslant 2$ and $K$ is transitive on points then $K$ contains no long root element.

Proof. Deny! Then the subgroup $N$ of $K$ generated by long root elements is irreducible by (7.1), and $N=\left\langle t^{N}\right\rangle$ for some long root element $t$ by (2.3). Thus, (2.4) applies. (This time, (1.1 viii, ix, $\mathrm{x})$ arise.)

Lemma 7.3. We may assume that $\chi \in 1_{B}^{G}$.

Proof. If $\left\langle\varphi, 1_{B}^{G}\right\rangle=1$, then Seitz's result (2.6) applies if $n \geqslant 2$. If $n=1$, then $K$ is transitive on points, so that (2.7), (2.8) and Dickson [12, Chapter 12] can be used to find $K$. In all cases, it is straightforward to check whether or not $K$ is maximal and $G$ has rank 3 on $G / K$. (The cases ( $1.1 \mathrm{v}$, vi, vii) occur here.)

Lemma 7.4. $\zeta \notin 1_{B}^{G}$, except perhaps if $G$ is $S p(2 n, 2)$ and $\zeta$ is $(n-1,1 \mid 0)$, $(n-1 \mid 1)$ or $(0 \mid n)$.

Proof. Suppose $\varphi \subset 1_{B}^{G}$. Then, excluding the stated possibilities for $\zeta$ in the case $\operatorname{Sp}(2 n, 2)$, we have $|G: K|=\varphi(1) \equiv 1(\bmod p)$ by $(4.7)$. By $(2.2), K$ is then reducible on $V$. (The cases $(1.1 \mathrm{i}$, ii) appear here.)

Convention. Throughout $\S \S 7,8$, we will assume that $\zeta \notin 1_{B}^{G}$. The inevitable $S p(2 n, 2)$ case will be dealt with in $\$ 9$.

LEMMA 7.5. $n>2$.

Proof. By $(7.3,4), K$ has 2 point-orbits, so that $(2.8 \mathrm{~b})$ applies when $n=1$. If $n=2$, then $\chi \in \theta_{1}$ or $\theta_{2}$, or $\chi=1_{B}^{G}-\theta_{1}-\theta_{2}+1_{G}$ (by 4.5(i) and Table II). Then using Table II in order to compute $\left\langle\varphi, 1_{B}^{G}\right\rangle$, we find that (5.13) or (5.14) applies. The rank on $G / K$ is straightforward but tedious to check geometrically. (The cases ( $1.1 \mathrm{v}$, vi, vii) occur here.) 
8. Transitivity. The most enjoyable part of the proof of (1.1) is the following crucial fact.

THEOREM 8.1. $K$ is transitive on points.

Proof. Assume that $K$ is not point-transitive. Then $\left\langle\chi, \theta_{1}\right\rangle=1$, so that $\chi=$ $(n-1, e \mid 1-e)$ for $e=0$ or 1 , and $K$ has just $\left\langle\varphi, \theta_{1}\right\rangle=2$ point-orbits $\Omega, \Omega^{\prime}$. By (4.5) and the argument following it, we have $\left\langle\varphi, \theta_{n-e}\right\rangle=2$ and $\left\langle\varphi, \theta_{1, n-e}\right\rangle=3$. Thus, $K$ has 2 orbits on $\left(1, n-e\right.$ )-flags (of each type if $G$ has Weyl group $W\left(D_{n}\right)$ ). There must then be an $n-e$-space whose stabilizer is transitive on its points; we may assume that $\Omega$ contains this $n-e$-space. By (2.13) there is an $n-e$-space $W$ not in $\Omega$ which contains an $n-e-1$-space of $\Omega$.

Since flags behave differently for types $B_{n}$ and $D_{n}$, we will consider these cases separately.

Type $B_{n}$. Each flag has the form $\left(V_{1}, V_{2}, \ldots, V_{n}\right)$, and there is an $i$ with $V_{i} \subseteq \Omega$, $V_{i+1} \nsubseteq \Omega$. Flags having different values of $i$ cannot be in the same $K$-orbit. Every value of $i$ between 0 and $\operatorname{dim} W=n-e$ can occur (using an $i$-space inside $W \cap \Omega$ if $i<n-e)$. However, by (4.5) we have $\left\langle\varphi, 1_{B}^{G}\right\rangle=1+n-e$, so any two flags with the same $i$ are in the same $K$-orbit. Set $i=0$, and apply (2.16) with $x=V_{1}$ in $\Omega^{\prime}$, in order to see that $K$ is listed in (1.1).

Type $D_{n}$. Each flag has the form $\left(V_{1}, \ldots, V_{n-2}, V_{n}, V_{n}^{\prime}\right)$, where $V_{n}$ and $V_{n}^{\prime}$ are $n$-spaces on an $n-1$-space $V_{n-1} \supset V_{n-2}$, and are in different $G$-orbits.

Once again, $K$ has $\left\langle\varphi, 1_{B}^{G}\right\rangle=1+n-e$ flag-orbits. As above, at least $n-2$ of these are accounted for by flags having $V_{n-2} \nsubseteq \Omega$. We will exhibit $3-e$ orbits of flags having $V_{n-2} \subseteq \Omega$.

By (4.5), there are $\left\langle\varphi, \theta_{2}\right\rangle=2$ orbits of lines: those contained in $\Omega$ and those meeting both $\Omega$ and $\Omega^{\prime}$ (the latter type can be seen in $W$ ). In particular, $\Omega^{\prime}$ contains no $n-1$-space. By (4.5), there are $\left\langle\varphi, \theta_{n-1}\right\rangle=3-e$ orbits of $n-1$-spaces and $\left\langle\varphi, \theta_{1, n-1}\right\rangle=4-e$ orbits of $(1, n-1)$-flags. There are thus $2-e$ orbits of $n-1$-spaces contained in $\Omega$, and one further orbit of $n-1$-spaces. One of the latter $n-1$-spaces contains an $n-2$-space in $W \cap \Omega$. Thus, each of the $3-e$ orbits of $n-1$-spaces has a member containing a $V_{n-2}$ contained in $\Omega$. This produces at least $3-e$ further flag-orbits.

As before, (2.16) applies, and completes the proof of (8.1).

Remark. Cases (iii)-(v) of $(2.16)$ clearly cannot occur in $(1.1)$. Also, $\Omega(7,3)$ has rank 4 on the cosets of $W\left(E_{7}\right)^{\prime}$, with 4 distinct subdegrees (Fischer [17, (15.3.16)]).

LeMma 8.2. $\chi \in \theta_{2}$ or $\theta_{3}$.

Proof. If $\chi \notin \theta_{2}, \theta_{3}$ then (by Tables II and III) $K$ is transitive on points, lines and planes, so that (6.4) applies. (Recall that the case of $\Omega^{+}(6, q)$ has been postponed until §10.)

Lemma 8.3. Either $n \leqslant 3$ or $V$ has type $\Omega^{+}(8, q)$.

Proof. Assume that $n \geqslant 4$ and $V$ does not have type $\Omega^{+}(8, q)$. Suppose first that $\chi \neq(n-2 \mid 2),(n-3 \mid 3)$. By Tables II and III, $\chi \notin \theta_{n}$, so that $K$ is transitive on 
$n$-spaces (of each type, if $G$ has type $D_{n}$ ). Again by those tables, $K$ has at most 2 orbits of lines and 3 orbits of $(1,2)$-flags. Then by $(6.5), K$ has 2 line-orbits. Thus, $\chi$ is $(n-2,2 \mid 0)$ or $(n-2,1 \mid 1)$. By Tables II and III, $K$ has at most 2 orbits of planes and $(1, n)$-flags (for each type of $n$-spaces in the case of $\Omega^{+}(2 n, q)$ ). If $\chi=(n-2,2 \mid 0)$ then $K$ has 3 orbits of $(2,3)$-flags. If $\chi=(n-2,1 \mid 1)$ then $K$ has 2 orbits of planes and (1,n)-flags (for each type of $n$-spaces), 4 orbits of $(2,3)$-flags and 7 orbits of $(1,2,3)$-flags; moreover, if $n=4$, there are 9 orbits of flags by Table II and (4.5 i). Thus, $\left(6.5^{\prime}\right)$ eliminates this possibility for $K$.

Now suppose that $\chi=(n-2 \mid 2)$. Again by Tables II and III, $K$ is point-transitive, has 2 orbits of lines, planes and (1,2)-flags, and 3 orbits of (2,3)-flags (except when $G$ has Weyl group $W\left(D_{5}\right)$, in which case there are 4 such orbits); and if $n=8$, then $K$ has 2 orbits of planes and 3 of $(3,4)$-flags. By (6.6), $G$ must have Weyl group $W\left(D_{5}\right)$. But here, Table III implies that $K$ has 2 orbits of each type of 5 -spaces, 3 orbits of planes and each type of $(1,5)$-flags, and 4 orbits of $(1,3)$-flags. Thus, $(6.8)$ eliminates this case.

Finally, suppose that $\chi=(n-3 \mid 3)$. We may assume that $G$ does not have Weyl group $W\left(D_{5}\right)$ (as otherwise we are back in the case $(3 \mid 2)$ by Table III). This time, $K$ is $(1,2)$-transitive, has 2 orbits of planes, $(1,2,3)$-flags and 4-spaces, and 3 orbits of $(3,4)$-flags (except that there are 4 such orbits if $G$ has Weyl group $W\left(D_{7}\right)$ ). Now (6.7) and (6.9) eliminate this case.

LEMMA 8.4. $n \neq 3$.

Proof. Suppose that $n=3$. By (8.1), (8.2) and Table II, $\chi=(1,1 \mid 1),(1 \mid 2)$ or $(0 \mid 3)$.

If $\chi=(1,1 \mid 1)$, then (by Table II) $K$ is transitive on points and planes, has 2 orbits of lines and (1,3)-flags and 3 orbits of $(1,2)$-flags. This is impossible by $(5.16)$.

If $\chi=(1 \mid 2)$, then $K$ is transitive on points, has 2 orbits of lines, planes and (1,2)-flags, 3 orbits of $(1,3)$-flags and 4 orbits of flags. Then (5.19) describes all possible groups $K$, and (5.20) discusses which produce rank 3 permutation representations.

Finally, if $\chi=(0 \mid 3)$, then $K$ is $(1,2)$-transitive and has 2 orbits of planes, (1,3)-flags and flags. This is impossible by (5.18).

LEMMA 8.5. $V$ does not have type $\Omega^{+}(8, q)$.

Proof. Suppose that $V$ has type $\Omega^{+}(8, q)$. Define $G^{+}=\Omega^{+}(8, q), K^{+}, \varphi^{+}$and $\chi^{+}$as in (4.1). Then $\left\langle\varphi^{+}-1-\chi^{+}, 1_{B}^{G^{+}}\right\rangle=0$.

If $\left\langle\chi^{+}, \theta_{2}\right\rangle=0$, then $K^{+}$is transitive on points and lines. This contradicts (5.21).

Thus, $\chi^{+} \subset \theta_{2}$. If $\chi^{+} \in \theta_{1}$, the proof of (8.1) applies with $K^{+}$in place of $K$. Consequently, $\chi^{+} \subseteq \theta_{2}-\theta_{1}$.

Suppose that $\chi^{+}=(2 \mid 2)^{ \pm}$. Let $\tau$ denote a triality automorphism of $P \Omega^{+}(8, q)$, and consider $\left(K^{+}\langle-1\rangle /\langle-1\rangle\right)^{\tau}$. This group behaves as in the preceding paragraph, if $\chi^{+\tau}=(3,1 \mid 0)$.

Thus, $\chi^{+}=(2,2 \mid 0),(2,1 \mid 1)$ or $(2 \mid 2)$. 
If $\chi^{+}=(2,2 \mid 0)$ then (by Table III) $K^{+}$is transitive on each class of $(1,4)$-flags, and has 2 orbits of lines, $(1,2)-,(1,3)$ - and $(2,3)$-flags and 3 of flags. Since $\chi^{+}$is $\tau$-invariant, $K^{+}$also has 2 orbits of each class of $(2,4)$ - and $(1,2,4)$-flags. By $(5.22)$, $K^{+}$is $W\left(E_{8}\right)^{\prime}$, embedded in $\Omega^{+}(8,3)$. Then $G$ has rank at least 4 on the cosets of $K$ (Fischer [17, (18.3.12)]).

If $\chi^{+}=(2,1 \mid 1)$ then $\chi^{+}$is again $\tau$-invariant. Now Table III and (5.23) yield a contradiction.

Finally, if $\chi^{+}=(2 \mid 2)$ then $G$ has type $B_{4}$, and $\chi=(2 \mid 2)$. This time, Table II and (5.25) yield a contradiction.

9. Proof of Theorem 1.1: Conclusion. In view of $\S \S 7,8$, the proof of $(1.1)$ is complete except for two situations: (A) $G=S p(2 n, 2)$ and $\varphi \subseteq 1_{B}^{G}$; and (B) $G$ has elements not arising from semilinear transformations of $V$. (Recall however, that the case of $V$ of type $\Omega^{+}(6, q)$ has been postponed until $\S 10$.)

(A) $G=S p(2 n, 2), n \geqslant 3$. (Of course, $S p(4,2) \cong S_{6}$.) By $(2.2),|G: K|$ is even. Thus, by (4.7), $\varphi=1+\chi+\zeta$ with $\zeta=(n-1,1 \mid 0),(n-1 \mid 1)$ or $(0 \mid n)$.

LEMMA 9.1. $2^{2 n-3} \mid \varphi(1)$. In particular,

(i) $2^{n-1}$ is the highest power of 2 dividing $\chi(1)$ if $\zeta$ is $(n-1,1 \mid 0)$ or $(n-1 \mid 1)$, and

(ii) 4 is the highest power of 2 dividing $\chi(1)$ if $\zeta=(0 \mid n)$.

Proof. Set $Q=C_{G}\left(x^{\perp} / x\right)$, and consider its action on the $\varphi(1)$ cosets of $K$. The orbit of $K$ has length $|Q: K \cap Q|$. By (7.1), (2.3) and [33], $K \cap Q$ does not contain long root elements of $\Omega(2 n+1,2)$. By (2.1), $|K \cap Q| \leqslant 4$. Thus, $|Q| / 4=2^{2 n-3}$ divides $\varphi(1)=1+\chi(1)+\zeta(1)$. By (4.7), $\varphi(1)=2^{n-1}\left(2^{n} \pm 1\right)+\chi(1)$ or $4\left(2^{2 n-3}+2^{n-2}+2^{n-3}+1\right) / 3+\chi(1)$. Assertions (i) and (ii) are now immediate.

LEMMA 9.2. If $n \geqslant 4$ then $\chi \in \theta_{23}$.

Proof. Suppose that $\chi \notin \theta_{23}$. If $\zeta=(0 \mid n)$ then (6.4) applies (by Table II). Thus, $\zeta=(n-1,1 \mid 0)$ or $(n-1 \mid 1)$. By Table II, $K$ has 2 orbits of points, lines and planes, and 3 orbits of $(1,2)-,(1,3)$ - and $(2,3)$-flags. Call the point-orbits $\Omega$ and $\Omega^{\prime}$. We may assume that $\Omega$ contains a plane. Then each line meets $\Omega^{\prime}$ in 0 or $k$ points, for some $k \geqslant 1$. By (2.11), $k=1$.

Let $x \in \Omega^{s}$. Then $K_{x}$ is transitive on the planes through $x$. If $r$ is a primitive divisor of $2^{n-2}+1$ (use $r=2^{3}-1$ if $n=5$ ), then $r|| K_{x} \mid$. Let $R \in S y l_{r} K_{x}$, and set $W=C_{V}(R), N=N_{K}(R)$ and $C=C_{N}\left(W^{\perp}\right)$ as in $\S 6$. Then $N^{W}$ is a subgroup of $S p(4,2)$ having at most 2 orbits of points and lines. Since $k=1$, it follows that $|W \cap \Omega|=5$ and $N^{W} \unrhd \Omega^{-}(4,2)$. Then $C^{W} \unrhd \Omega^{-}(4,2)$ as well.

Set $Q=C_{G}\left(x^{\perp} / x\right)$. Then $|C \cap Q|=4$. Since $K_{x}^{x^{\perp} / x}$ is point-transitive, (2.1) implies that $K \geqslant Q$, which is ridiculous.

LEMMA 9.3. $n \leqslant 4$.

Proof. Suppose that $n \geqslant 5$. Using Mayer [35, (1.1)], we find that $\theta_{13}$ has 15 irreducible constituents, 12 of which have even degree. Of these, only the character 
$(n-3 \mid 3), n \geqslant 6$, has degree $\equiv 4(\bmod 8)$, by (4.8). Suppose that $\zeta=(0 \mid n)$. By $(9.1), \chi=(n-3 \mid 3)$. By $(4.8)$,

$|G: K|=4\left(2^{2 n-3}+2^{n-2}+2^{n-3}+1\right) / 3+4\left(2^{2 n}-1\right)\left(2^{2 n-2}-1\right)\left(2^{n-2}+1\right) / 315$ is not divisible by $2^{2 n-3}$. Thus, $\zeta \neq(0 \mid n)$ by $(9.1)$.

By (9.2), $\chi \in \theta_{23}$, and by $(9.1), \chi(1) \equiv 2^{n-1}\left(\bmod 2^{n}\right)$. By $(4.8), \chi$ must be $\left(3,1 \mid 1^{2}\right),\left(3,1^{2} \mid 1\right)$ or $(2 \mid 2,1)$. However, $1+\zeta(1)+\chi(1) \nmid|G|$ in each of these cases.

Proposition 9.4. There is no counterexample to (1.1) when $G=S p(2 n, 2)$.

Proof. We must eliminate the cases $n=3,4$. Let $n=4$. As in (9.3), we find that $\varphi$ is $(4 \mid 0)+(3,1 \mid 0)+\left(2,1^{2} \mid 0\right)$ or $(4 \mid 0)+(3 \mid 1)+\left(2,1^{2} \mid 0\right)$. In neither case is there a divisor $d$ of $|G|$ such that $\varphi(1) d(\varphi(1)-d-1) / \chi(1) \zeta(1)$ is a square, contrary to Frame's theorem $[62,(30.1)]$.

Similarly, if $n=3$, a routine calculation shows that there are only 3 characters $\varphi \subseteq 1_{B}^{G}$ such that $\langle\varphi, 1\rangle=1,\langle\varphi, \varphi\rangle=3$ and $\varphi(1)|| G \mid$. Once again, none of these satisfies Frame's theorem. This proves (9.4).

(B) Graph automorphisms. There are two cases to consider: $S p\left(4,2^{e}\right)$ and $P \Omega^{+}(8, q)$. (The graph automorphisms of $\Omega^{+}(2 n, q), n>4$, of order 2 were already considered when we discussed $O^{+}(2 n, q)$.)

As usual, we have $\varphi=1+\chi+\zeta$. Define $K^{+}, \varphi^{+}, \chi^{+}, \zeta^{+}$and $B$ as in (4.1). By (2.6), we may assume that $\left\langle\varphi^{+}, 1_{B}^{G^{+}}\right\rangle>1$. By (4.1d), we may assume that $\chi^{+} \subseteq 1_{B}^{G^{+}}$. If $\varphi^{+} \subseteq 1_{B}^{G^{+}}$then (2.2) applies by (4.7), except in the easy case $S p(4,2)$. We may thus also assume that $\left\langle\zeta^{+}, 1_{B}^{G^{+}}\right\rangle=0$.

Suppose that $S p\left(4,2^{e}\right)<G \leqslant$ Aut $S p\left(4,2^{e}\right)$. If $\chi^{+}$is irreducible, use (5.6). If $\chi^{+}$is reducible then $\chi^{+}=(1,1 \mid 0)+(0 \mid 2)$. Here, $K^{+}$has 3 point-, line- and flag-orbits. No such group exists. (If $L$ is any line then $\left(K^{+}\right)_{L}^{L}$ would be transitive, and hence $K^{+}$would be point-transitive.)

Next suppose that $P \Omega^{+}(8, q)<G \leqslant$ Aut $P \Omega^{+}(8, q)$. If $\chi^{+}$is irreducible, the arguments in $\S \S 7,8$ apply with $K^{+}$replacing $K$. We may thus assume that $\chi^{+}$is reducible. By Table III, $\theta_{2}$ is $G$-invariant, so $\chi^{+} \subseteq \theta_{2}$ by (5.22). Again by Table III, $\chi^{+}$must be a sum of 2 or 3 of the characters $(3,1 \mid 0),(2 \mid 2)^{+}$and $(2 \mid 2)^{-}$.

By $(5.25)$, the case $\chi^{+}=(3,1 \mid 0)+(2 \mid 2)^{+}+(2 \mid 2)^{-}$is impossible. If $\chi^{+}$has just 2 irreducible constituents, we can use Aut $P \Omega^{+}(8, q)$ in order to assume that $\chi^{+}=(2 \mid 2)^{+}+(2 \mid 2)^{-}$. In this case, $G \leqslant P \Gamma O^{+}(8, q)$, and $G$ has type $B_{4}$. Thus, this case was handled in $\S \S 7,8$.

This completes the proof of (1.1).

10. Proof of Theorem 1.2. The more complicated results appearing in $\$ \S 2-5$ are not required for (1.2), as the following remarks indicate.

REMARK 10.1. We will require (3.8) when $\theta_{i}$ is regarded as the permutation character on $i$-spaces of a group lying between $\operatorname{Si}(n, q)$ and $\Gamma L(n, q)$, and when $\theta_{i j}$, etc., are viewed similarly. The assertions in (3.8) can then be proved directly and easily as follows. For (i), consider $\left\langle\theta_{i-1}, \theta_{i-1}\right\rangle,\left\langle\theta_{i}, \theta_{i-1}\right\rangle$ and $\left\langle\theta_{i}, \theta_{i}\right\rangle$. Statements (ii) and (iii) are especially easy. For (iv)-(vi), proceed as in the following example. In order to compute $\left\langle\theta_{3}-\theta_{2}, \theta_{34}\right\rangle$, calculate the number of orbits of the stabilizer of a $(3,4)$-flag in its actions on both 3 -spaces and 2 -spaces, and subtract. 
REMARK 10.2. From $\S 2$, we will require the relatively elementary results $(2.7,2.15)$. All transitivity results in $\$ 5$ will be avoided. Instead, we will refer to [23], [58], [46], [31], [32] for transitivity statements. It should be noted that the results quoted from [32] are merely simple cases of Perin's method, and should be straightforward exercises for anyone who has read [46] or examined $(6.1,2)$.

We now begin the proof of (1.2). We are given $\operatorname{PSL}(n, q) \leqslant G \leqslant$ Aut $\operatorname{PSL}(n, q)$ and $K \leqslant G$. Define $G^{+}=G \cap P \Gamma L(n, q), K^{+}, \varphi, \varphi^{+}, \chi, \chi^{+}, \zeta, \zeta^{+}$and $B$ as in (4.1).

If $\left\langle\varphi^{+}, 1_{B}^{G^{+}}\right\rangle=1$ then $K^{+}$is flag-transitive. Thus, Higman [23] can be applied if $n>2$, while Dickson [12, Chapter 12] provides a short list to check if $n=2$.

Similarly, if $n=2$ and $\left\langle\varphi, 1_{B}^{G}\right\rangle \neq 1$ then $\left\langle\varphi, 1_{B}^{G}\right\rangle=2$ and $K$ has exactly 2 point-orbits. This time a longer list of possibilities for $K \cap S L(2, q)$ can be obtained from Dickson [12, Chapter 12]. In most cases there are elementary geometric arguments showing that the rank of $G$ on $G / K$ is greater than 3 . The remaining ones are dealt with by arithmetic arguments. (For example, $|G: K|-1-q$ is divisible by the degree of an irreducible character of $\operatorname{PSL}(2, q)$, namely, $q \pm 1$ or $\frac{1}{2}(q \pm 1)$.)

If $\varphi^{+} \subseteq 1_{B}^{G^{+}}$, then $\left|G^{+}: K^{+}\right| \equiv 1(\bmod p)$ by (4.7). (This simple case of (4.7) is implicit in Steinberg [55]; cf. [9, (5.9)].) Thus, (2.2) applies.

In view of (4.1), we may assume that $\chi^{+} \subseteq 1_{B}^{G^{+}}$, and $\left\langle\zeta^{+}, 1_{B}^{G^{+}}\right\rangle=0$. Note that $\chi^{+}$ is irreducible. For, by $(4.1 \mathrm{c}), \chi^{+}$is a sum of characters conjugate in $G$, while each irreducible constituents of $1_{B}^{G^{+}}$is $G$-invariant. (In order to see this, note that the permutation character of $G^{+}$on $\left(i_{1}, \ldots, i_{t}\right)$ - and $\left(n-i_{t}, \ldots, n-i_{1}\right)$-flags coincide, as in (4.3). Since every constituent of $1_{B}^{G^{+}}$is a linear combination of such permutation characters by Steinberg [55] or (4.2), this implies the desired invariance.)

At this stage, we no longer require information of a rank 3 nature. All that is needed is the following more general result (in which our notation has been altered somewhat).

THEOREM 10.3. Let $S L(n, q) \leqslant G \leqslant \Gamma L(n, q)$, where $n>2$. Let $B$ be a Borel subgroup of $G$, and let $K<G$. Assume that $1_{B}^{G}$ and $1_{K}^{G}$ have a common nonprincipal irreducible constituent $\chi$ such that $\left\langle 1_{K}^{G}-1-\chi, 1_{B}^{G}\right\rangle=0$. Then one of the following holds ( with the obvious embedding):

(i) $K$ fixes a point or hyperplane;

(ii) $K \unrhd 3 \cdot A_{6}$, inside $\Gamma L(3,4)$;

(iii) $K \unrhd S p(4, q)$, inside $\Gamma L(4, q)$;

(iv) $K=A_{6}$, inside $\Gamma L(4,2)$; or

(v) $K \triangleright S L\left(2, q^{2}\right)$, inside $\Gamma L(4, q)$.

Remark. In the context of (1.2), only (ii), $S p(4,2)$ and $S p(4,3)$ can arise, corresponding to (1.2 iv, v, vi). For, (i) and (iv) clearly cannot occur, by primitivity. Examples (iii) and (v) are best viewed by passing to $\mathrm{\Gamma O}^{+}(6, q)$, where they correspond to subgroups of the stabilizer of an anisotropic 1- or 2-space; from this perspective, it is easy to check for the rank 3 property.

Proof of (10.3). We will proceed in several steps. Let $V$ denote the underlying vector space. 
(I) Suppose that $\chi=\theta_{1}-1$ is the reflection character. By (3.8), $K$ then has 2 orbits of points and hyperplanes, and 3 orbits of $(1, n-1)$-flags. Thus, $K$ has a point-orbit $\Omega$ containing a hyperplane. By (4.5) (or a simple argument as in (10.2)), there are $1+(n-1)$ flag-orbits. Each flag has the form $\left(V_{1}, \ldots, V_{n-1}\right)$ with $V_{i} \subset \Omega$ and $V_{i+1} \nsubseteq \Omega$, for some $i$. This accounts for $n$ possibilities, one for each $i$ between 0 and $n-1$. Thus, if $x \notin \Omega$ then $K_{x}$ is flag-transitive on $V / x$. By (2.15), (i) or (ii) holds.

(II) From now on, we may assume that $K$ is transitive on points. By ( $2.7 \mathrm{i}), n \geqslant 4$.

Assume that $\chi \neq \theta_{2}-\theta_{1}$. Then $K$ is line-transitive. By Kantor [31], [32], $K$ is 2 -transitive on points and $n \geqslant 10$. (The only possible counterexample given in [31] is a Sylow 31-normalizer in $S L(5,2)$, which has 7 orbits of $(1,3)$-flags.) By Perin [46], $K$ is intransitive on 4-spaces, and even on planes if $q>2$. Thus, $\chi$ is $\theta_{3}-\theta_{2}$ or $\theta_{4}-\theta_{3}$.

Suppose $\chi=\theta_{3}-\theta_{2}$. By (3.8), $K$ has 2 plane-orbits and 3 orbits of $(3,4)$-flags. Thus, there is a plane $E$ such that $K_{E}$ is transitive on the $\left(q^{n-3}-1\right) /(q-1)$ points of $V / E$. By (2.18), $q^{n-3}-1$ has a primitive divisor. Thus, $K \geqslant S L(V)$ by [32, (5.2)].

Similarly, if $\chi=\theta_{4}-\theta_{3}$ and $q=2$ then $2^{n-4}-1|| K \mid$, and [32] again shows that $K \geqslant S L(V)$.

(III) Thus, $\chi=\theta_{2}-\theta_{1}$.

Suppose $n=4$. By (3.8) and (5.5), (iii), (iv) or (v) holds.

This completes the proof of $(1.1)$ when $K \leqslant \Gamma O^{+}(6, q)$.

Remark 10.4. In view of remarks $(10.1,2)$, it seems desirable to outline a more direct argument when $n=4$, in order to avoid using (5.5). Note that $K$ is transitive on points and planes, has 2 orbits of lines, $(1,2)$ - and (1,3)-flags, and 3 orbits of flags. Let $E$ be a plane. By (2.7), $K_{E}^{E}$ fixes a point, line or (if $q=4$ ) a hyperoval. If $K_{E}^{E}$ fixes a hyperoval, then $\left|Z\left(K_{E}^{E}\right)\right|=3$, and Wagner [60] applies.

Suppose that $K_{E}^{E}$ fixes a point $x$. Then $K_{E}=K_{x}$. Here, $x \leftrightarrow E$ defines a symplectic polarity. (For, $K_{x}$ is transitive on the $q+1$ lines of $E$ through $x$, and on the remaining $q^{2}$ lines through $x$. Let $\langle x, y\rangle$ be one of the latter lines. Then $K_{x,\langle x, y\rangle}$ is still transitive on the aforementioned $q+1$ lines, by (2.12). Thus, $K_{x y}$ cannot fix any plane on both $x$ and $y$.) Since $K_{x}^{E}$ has just 2 line-orbits, it contains all transvections with direction $x$. Thus, if $x \in L \subset E$ then $K_{x}^{L}$ is transitive on $L-x$. Now $K_{L}^{L} \geqslant S L(2, q)$. Also, $q|| C_{K}(L) \mid$. Applying $(2.1)$ to $\Gamma O(5, q)$, we find that $C_{K}(L) \geqslant O_{p}\left(G_{L}\right)$ if $q>2$. Thus, (iii) or (iv) holds.

Next suppose that $K_{E}^{E}$ fixes a line $L$. Then $K_{E}^{E}$ is transitive on the remaining lines of $E$, so that $L$ is the only member of $L^{K}$ lying in $E$. Consequently, $\left|L^{K}\right|=q^{2}+1$, and $L^{K}$ partitions the points of $V$. Note that $O_{p}\left(C_{K}(L)_{E}\right)$ is transitive on $E-L$, and hence on $L^{K}-\{L\}$. It follows that $L^{K}$ is uniquely determined up to $G L(4, q)$-conjugacy $[10$, pp. 130-131]. Hence, (v) holds.

(IV) From now on we will assume that $n \geqslant 5$ in order to eventually obtain a contradiction.

Since $\chi=\theta_{2}-\theta_{1},(3.8)$ implies that there are 2 orbits of lines, planes and $(1,2)$-flags, 3 orbits of $(2,3)$-flags and 4 orbits of $(1,2,3)$-flags. Thus, there is a plane $E$ such that $K_{E}^{E}$ is transitive, and a plane $E^{\prime}$ such that $K_{E^{\prime}}^{E^{\prime}}$ has 2 point-orbits. Then $K_{E^{\prime}}^{E^{\prime}}$ has at least 3 flag-orbits, and hence $K_{E}^{E}$ is flag-transitive. 
There is a line $L$ contained in $E^{\prime}$ but contained in no member of $E^{K}$. Then $K_{L}$ is transitive on the points and hyperplanes of $V / L$. Consequently, if $H$ is a hyperplane, then $K_{H}$ is transitive on those members of $L^{K}$ lying in $H$.

Similarly, there are 2 orbits of $(1,2,3)$-flags on the form $\left(x, L, E^{\prime}\right)$, and hence also 2 of the form $(x, L, H)$. Thus, $K_{L H}^{L}$ has 2 point-orbits.

By (3.8), $K_{H}^{H}$ has 2 point-orbits (called $\Omega$ and $\Omega^{\prime}$ ), 3 line-orbits and 4 orbits of (1,2)-flags. Thus, $L \cap \Omega$ and $L \cap \Omega^{\prime}$ are the orbits of $K_{L H}^{L}$, and only lines in $L^{K}$ can meet both $\Omega$ and $\Omega^{\prime}$.

Again by (3.8), $K$ has 3 orbits of $(1,3)$-flags and at most 5 orbits of $(1,3,4)$-flags. Thus, $K_{x T}$ is point-transitive on $V / T$ for some $(1,3)$-flag $(x, T)$.

(V) Assume that $K_{x}$ fixes a proper subspace $U(x) \supset x$. Since $K_{L}$ is transitive on $V / L, U(x)$ is either a line or a hyperplane.

If $U(x)$ is a line, then $U(x)=L$ and no two members of $U(x)^{K}$ meet nontrivially. Then $T$ meets $q^{2}$ or $q^{2}+q+1$ members of $L^{K}$ in just one point, and $K_{x T}$ has a point-orbit on $V / T$ of length at most $q^{2}$. Since $K_{x T}$ is transitive on $V / T$, this is impossible.

Thus, $U(x)$ is a hyperplane. As in (10.4), $x \leftrightarrow U(x)$ defines a symplectic polarity. However, $K_{T}^{V / T}$ cannot be transitive in a symplectic geometry.

Thus, $K_{x}^{V / x}$ is irreducible. Dually, so is $K_{H}^{H}$.

(VI) That $n \neq 5$ follows from (5.5).

Alternatively, since $K_{E}^{E}$ is flag-transitive, $K_{E H}^{E}$ is transitive whenever $H \supset E$ (by (2.7 i)). Thus, (2.15) shows that $K_{H}^{H}$ cannot be irreducible.

(VII) Thus, $n \geqslant 6$. By (3.8), $K_{H}^{H}$ has 3 plane-orbits, 5 orbits of $(1,3)$-flags and 7 orbits of $(1,2,3)$-flags. By $(2.7$ iii), two of these plane-orbits produce subgroups of $\Gamma L(3, q)$ having 2 point-orbits and 3 flag-orbits, while the third produces a flag-transitive subgroup.

We may assume that $L \subset E^{\prime} \subset H$. By (2.7 ii), $K_{L E^{\prime} H}^{L}$ has orbit lengths 1 and $q$, except perhaps if $q=4$ and the lengths are 2 and 3 .

(VIII) If $|L \cap \Omega|=1$, then any line meeting $\Omega$ twice must be contained in $\Omega$. (For, we have seen that $K_{H}^{H}$ has a unique orbit of lines meeting both $\Omega$ and $\Omega^{\prime}$.) Thus, $\Omega$ is a subspace, and this contradicts (V).

Consequently, $q=4$ and $K_{E^{\prime}}^{K^{\prime}} \unrhd 3 \cdot A_{6}$. Also, $K_{E}^{E} \geqslant S L(3,4)$. Let $r$ be a primitive divisor of $4^{n-3}-1$, and let $R \in S y l_{r} K_{x T}$. Then $R$ centralizes $T$, and $N_{K}(R)^{T}=K_{T}^{T}$ by the Frattini argument. It follows that $K_{C}([V, R])^{T}$ contains transvections, and hence so does $K$, which is ridiculous.

This contradiction completes the proof of (10.3), and hence also of (1.2).

\section{Remarks concerning Theorems 1.1 and 1.2.}

(A) Just as in the case of (1.2) and (10.1), the proof of (1.1) did not make full use of the rank 3 hypothesis. The crux of the argument was the following companion to Seitz's flag-transitive theorem (2.6).

THEOREM 11.1. Suppose that $V$ has rank at least 2, but is not of type $\Omega^{+}(4, q)$ or $\Omega^{+}(6, q)$. Let $\operatorname{Chev}(V) \leqslant G \leqslant \Gamma(V)$, and let $K<G$. Assume that there is an irreducible character $\chi$ common to $1_{K}^{G}$ and $1_{B}^{G}$ such that $\left\langle 1_{K}^{G}-\chi, 1_{B}^{G}\right\rangle=1$, where $B$ is a Borel 
subgroup of $G$. Then one of the following holds, with $K$ embedded in $\Gamma(V)$ in the natural manner:

(i) $K \geqslant \operatorname{Chev}(H)^{\prime}$ for a nonsingular hyperplane $H$, where $V$ is orthogonal or unitary and $\chi=(n-1 \mid 1)$ or $(n-1,1 \mid 0)$;

(ii) $V, K$ and $\chi$ are given in Table $\mathrm{V}$; or

(iii) $K$ arises from Table $\mathrm{V}$ via one of the isomorphisms $\operatorname{PSp}(4, q) \cong P \Omega(5, q)$, $P S U(4, q) \cong P \Omega^{-}(6, q)$, or $\Omega\left(2 n+1,2^{i}\right) \cong S p\left(2 n, 2^{i}\right)$.

The proof proceeds exactly as in $\S \S 7,8$. However, more care is needed in checking the examples occurring in the course of the proof. This is accomplished as follows. $K$ has $\left(1_{K}^{G}, \theta_{1}\right) \leqslant 2$ point-orbits; this eliminates most instances. In order to then verify that a prospective example behaves as desired, one computes the number of flag-orbits geometrically. If $K$ is not point-transitive, (4.5 i) is used; for example, (11.1 i) is checked by reversing the proof of (8.1).

(B) Imprimitive rank 3 groups. In order to underscore the previous remark we observe that Theorems 1.1 and 1.2 can now be generalized to include imprimitive rank 3 groups. For example, if $G \leqslant \Gamma(V)$ as in (1.1) and $G$ acts on $G / K$ as an imprimitive rank 3 group, then one of (2.6), (11.1) or (2.2) applies depending upon the number of parabolic characters in the associated permutation character. Comparing the resulting list of groups with those in the main theorem of [9] (and using the fact that $G$ acts 2-transitively on a system of imprimitivity) we have:

TheOREM 11.2. Let $G$ be as in (11.1) and let $K \leqslant G$. Assume that $G$ acts as an imprimitive rank 3 group on $G / K$. Then $\Omega^{ \pm}(2 n, 2)=K<S p(2 n, 2)=G$.

In order to see that these are rank 3 representations, view $\operatorname{Sp}(2 n, 2)$ as the stabilizer of a nonsingular point in $O^{ \pm}(2 n+2,2)$ and let $K$ be the centralizer of a nonsingular 2-space on $x$ not in $x^{\perp}$. In a similar manner we obtain:

THEOREM 11.3. Let $G$ be as in (10.1) and let $K \leqslant G$. Assume that $G$ acts as an imprimitive rank 3 group on $G / K$. Then either $K=A_{7}<S_{8} \cong$ Aut $\operatorname{PSL}(4,2)$ or $K$ has index 2 in the stabilizer of a point or hyperplane.

(C) Other Chevalley groups. It would be worthwhile to extend the methods presented here to the other Chevalley groups. Clearly, transitivity questions would have to be handled somewhat differently, due to the seeming lack of a convenient module. The known examples of rank 3 representations are as follows: (i) $E_{6}(q)$ on either of two classes of parabolic subgroups; (ii) $G_{2}(2)=P S U(3,3) \cdot 2$ as in (1.1 x); and (iii) $G_{2}(4)$ on a class of Hall-Janko subgroups (Suzuki [56]).

(D) Although we have dealt primarily with $\Gamma(V)$, it may be helpful to observe that our main theorems could have been proven by working entirely inside $\operatorname{Chev}(V)$. While we have chosen the present exposition hoping that some of our numerous intermediate technical results might find greater application, such "linearization" might be necessary to deal with the other Chevalley groups. This kind of restriction is facilitated by (4.1), (4.4) and the following lemma. 
TABLE V

\begin{tabular}{|c|c|c|c|}
\hline Chev(V) & A normal subgroup of $K$ & $\underline{x}$ & Reference \\
\hline \multirow[t]{2}{*}{$\Omega^{+}(8, q)$} & $\begin{array}{l}\Omega(7, q) \text { or } 2 \cdot \Omega(7, q) \\
A_{9}, q=2\end{array}$ & $\begin{array}{l}(2 \mid 2)^{ \pm} \text {or } \\
(3,1 \mid 0)\end{array}$ & $\begin{array}{l}(2.16) \text { and } \\
\text { triality }\end{array}$ \\
\hline & $2 \cdot \Omega^{+}(8,2), q=3$ & $(2,2 \mid 0)$ & $(5.22)$ \\
\hline \multirow[t]{2}{*}{$\Omega(7, q)$} & $\begin{array}{l}G_{2}(q)^{\prime} \\
P \Gamma L\left(2, q^{3}\right), q=2 \text { or } 8\end{array}$ & $(1 \mid 2)$ & $(5.19)$ \\
\hline & $S p(6,2), q=3$ & $(2,1 \mid 0)$ & $(2.16)$ \\
\hline$S U(6,2)$ & $3 \cdot P S U(4,3)$ & $(2 \mid 1)$ & $(2.16)$ \\
\hline$S U(5,2)$ & $Z_{3}^{4} \rtimes A_{5}$ & $(1 \mid 1)$ & $(5.14)$ \\
\hline \multirow[t]{3}{*}{$S U(4, q)$} & $\begin{array}{l}S p(4, q)^{\prime} \\
4 \cdot Z_{2}^{4} \cdot P S L(2,5) \text { or } 4 \cdot Z_{2}^{4} \cdot A_{6}, q=3\end{array}$ & $(1 \mid 1)$ & $(5.14)$ \\
\hline & $4 \cdot P S L(3,4), q=3$ & $(0 \mid 2)$ & $(5.13)$ \\
\hline & $\begin{array}{l}Z_{2}^{3} \rtimes A_{4}, q=2 \\
S U(3,2)^{\prime \prime} \rtimes Z_{8}, q=2\end{array}$ & $(1,1 \mid 0)$ & $(5.13)$ \\
\hline \multirow[t]{3}{*}{$S p(4, q)$} & $\begin{array}{l}S L(2, q) \times S L(2, q) \\
\Omega^{+}(4, q), q \text { even } \\
S L(2,3)^{\prime} \times S L(2,3)^{\prime}, q=3 \\
2 \cdot Z_{2}^{4} \cdot A_{4}, q=3 \\
Z_{3}^{2}, q=3\end{array}$ & $(1 \mid 1)$ & $(5.14)$ \\
\hline & $\begin{array}{l}S p\left(2, q^{2}\right) \\
2 \cdot Z_{2}^{4} \rtimes D_{10}, S L(2,5), q=3\end{array}$ & $(0 \mid 2)$ & $(5.13)$ \\
\hline & $\Omega^{-}(4, q), q$ even & $(1,1 \mid 0)$ & $(5.13)$ \\
\hline
\end{tabular}

Lemma 11.4. Suppose $G \unrhd G^{+}$and $K^{+}=K \cap G^{+}$for some $K \leqslant G$. Suppose $\chi$ is an irreducible character of $G$ such that $\left\langle\chi, 1_{K}^{G}\right\rangle \neq 0$. If $\chi^{+}=\left.\chi\right|_{G^{+}}$is irreducible then

$$
\left\langle 1_{K}^{G}, \chi\right\rangle=\left\langle 1_{K^{+}}^{G^{+}}, \chi^{+}\right\rangle \text {. }
$$


Proof. By Frobenius reciprocity and the fact that $1_{K}^{G}=\left(1_{K}^{G^{+} K}\right)^{G}$, it suffices to consider the case $G=G^{+} K$. In this case,

$$
\left\langle 1_{K^{+}}^{G^{+}}, \chi^{+}\right\rangle=\left\langle 1_{K}^{G^{+}}, \chi\right\rangle=\left\langle\sum \omega(1) \omega^{G}, \chi\right\rangle=\sum \omega(1)\left\langle\omega,\left.\chi\right|_{H}\right\rangle
$$

where the summations range over the irreducible characters $\omega$ of $K$ with kernel containing $K^{+}$. The irreducible constituents of $\left(\chi^{+}\right)^{G}$ are of the form $\chi^{+} \varphi$ where $\varphi$ is an irreducible character of $K$ with kernel containing $K^{+}$(Isaacs [28, (6.17)]). Since $\chi$ is an extension of $\chi^{+}$, Frobenius reciprocity implies $\chi=\chi^{+} \varphi$ for some such $\varphi$. However, an easy calculation shows

$$
\left\langle\left.\chi\right|_{K}, \omega\right\rangle=\left\langle\left.\chi\right|_{K^{+}}, 1_{K^{+}}\right\rangle\langle\varphi, \omega\rangle
$$

for any $\omega$ as above, and $0 \neq\left\langle\chi, 1_{K}^{G}\right\rangle=\left\langle\left.\chi\right|_{K}, 1_{K}\right\rangle\left\langle\theta, 1_{K}\right\rangle$ by hypothesis and the preceding equation. Therefore $\varphi=1_{K}$, and the same equation shows $\left\langle\left.\chi\right|_{K}, \omega\right\rangle=\left\langle\left.\chi\right|_{K}, 1_{K}\right\rangle \delta_{\omega, 1_{H}}$. Thus

$$
\left\langle 1_{K^{+}}^{G}, \chi^{+}\right\rangle=\sum \omega(1)\left\langle\omega,\left.\chi\right|_{H}\right\rangle=\left\langle 1_{K}^{G}, \chi\right\rangle,
$$

as desired.

12. Proof of (1.3). Since $K$ is $(1,2)$-transitive, by (2.6) and (5.15) we may assume that $d \geqslant 7$. Set $Q=O_{p}\left(\Gamma O(V)_{x}\right)$; we may also assume that $K \cap Q=1$, by (2.1) and (2.4).

Let $r$ be a primitive divisor of $q^{m-2} \pm 1$, for $V$ of type $\Omega^{ \pm}(2 m, q)$ or $\Omega(2 m-1, q)$; define $R \in S y l_{r} K_{x}, W, N$ and $C$ as in $\S 6$. By (2.18), $r$ exists except in the cases $\Omega^{+}(10,2), \Omega^{-}(16,2)$, and $\Omega^{-}(8, q)$ with $q$ a Mersenne prime, all of which we temporarily exclude. By Table IV, $R \neq 1$.

Note that $W$ contains no line, while $N^{W}$ is transitive on ordered pairs of points. Thus, $N^{W} \unrhd \Omega^{-}(4, q)$ or $\Omega(3, q)$, while $N^{W^{\perp}}$ is isomorphic to a subgroup of $\Gamma O^{*}\left(2, q^{m-2}\right)$. Consequently, unless $N^{W} \unrhd \Omega(3,3)$, we find that $C^{W} \geqslant \Omega^{-}(4, q)$, and hence that $C \cap Q \neq 1$.

Thus, $K \leqslant \Gamma O(2 m-1,3)$. Here, $\left|O_{2}\left(C^{W}\right)\right| \geqslant 4$. Let $b$ be a nonsingular point of $W$ whose corresponding reflection $t$ fixes 2 points of $W$. Set $H=\left\langle K_{b}, C\right\rangle\langle t\rangle$. Since $\left|b^{\Gamma O(V)} \cap\left\langle x_{1}, x_{2}\right\rangle\right|=1$ for any pair of nonperpendicular points $x_{1}, x_{2},\langle t\rangle K_{b}$ is transitive on the set of points not in $b^{\perp}$. Since $C$ moves $b$, it follows that $b^{h} \notin b^{\perp}$ for some $h \in H$. But then $t t^{h} \in K$, and $t t^{h}$ is in the conjugate $O_{3}\left(\Gamma O(V)_{x^{\prime}}\right)$ of $Q$ (where $\left.x^{\prime}=\operatorname{rad}\left\langle b, b^{h}\right\rangle\right)$, contrary to our assumption.

We now turn to the excluded cases. If $K \leqslant \Gamma O^{-}(16,2)$, use $r=7$ and proceed as before. Note that $R$ fixes a 6-space of $W^{\perp}$, and hence that $N^{W^{\perp}}$ is contained in $\Gamma \mathrm{O}^{+}\left(4,2^{3}\right)$. However, $\left(N^{W}\right)^{(\infty)}=A_{5}$ is not contained in the latter group.

If $K \leqslant \Gamma O^{+}(10,2)$, let $R_{1} \in \mathrm{Syl}_{3} K_{x}$. Since $x^{\perp} / x$ has $\left(2^{4}-1\right)\left(2^{3}+1\right)$ points, $\left|R_{1}\right| \geqslant 3^{3}$. However, $\Gamma O^{+}(8,2)$ has no element of order $3^{3}$, so $R_{1}$ cannot be fixed-point-free on $x^{\perp} / x$. Let $R$ be a 3-group maximal with respect to having $\operatorname{dim} W>2$, where $W=C_{V}(R)$; define $N$ and $C$ as usual. If $W$ has type $\Omega^{-}(4,2)$, the usual approach works. Since an $\Omega^{+}(6,2)$ space has $\left(2^{3}-1\right)\left(2^{2}+1\right)$ points, $W$ cannot have type $\Omega^{+}(4,2)$. If $\operatorname{dim} W>4$, it follows similarly that $W$ has type $\Omega^{+}(6,2)$ or $\Omega^{-}(8,2)$. By Sylow's theorem, $N^{W}$ is then line-transitive, so that 7||$N^{W} \mid$ and we can proceed as in the preceding paragraph. 
Finally, suppose that $K \leqslant \Gamma O^{-}(8, q)$ with $q$ Mersenne. If $L$ is a line, clearly $K_{L}^{L} \geqslant S L(2, q)$. We may assume that $-1 \in K$. If $R \in S y l_{2} C_{K}(L) \cap S L(8, q)$, then $R \neq 1$. Define $W, N$ and $C$ as usual. Then $N^{W}$ is $(1,2)$-transitive, and $N_{L}^{L} \geqslant S L(2, q)$ by the Frattini argument. Clearly, $\operatorname{dim} W=4,5$ or 6 . If $\operatorname{dim} W=6$, then $C \cap Q \neq 1$ by (2.6) and (5.15). If $\operatorname{dim} W=5$ then $q=3$ or $C \cap Q \neq 1$ by (2.6). If $W$ has type $\Omega^{+}(4, q)$ then $N^{W} \geqslant O^{+}(4, q)$ while $N^{W^{\perp}}$ normalizes the 2-group $R^{W^{\perp}} \leqslant \Gamma O^{-}(4, q)$; consequently, $q=3$ (as otherwise, $C^{W} \geqslant \Omega^{+}(4, q)$ and $C \cap Q \neq 1$ ). We can now introduce a reflection as before in order to complete the proof of (1.3).

REMARK. It should be noted that the linearity assumed by Perin was as superfluous for his arguments as it was for ours.

\section{REFERENCES}

1. J. L. Alperin and D. Gorenstein, A vanishing theorem for cohomology, Proc. Amer. Math. Soc. 32 (1972), 87-88.

2. E. Bannai, Maximal subgroups of low rank of finite symmetric and alternating groups, J. Fac. Sci. Univ. Tokyo 18 (1972), 475-486.

3. R. W. Carter, Simple groups of Lie type, Wiley, London, 1972.

4. E. Cline, B. Parshall and L. Scott, Cohomology of finite groups of Lie type. I, Inst. Hautes Études Sci. Publ. Math. 45 (1975), 169-191; II, J. Algebra 45 (1977), 182-198.

5. A. M. Cohen, Rank 3 permutation representations of finite complex reflection groups, Memorandum 154, Technische Hogeschool, Twente, The Netherlands, 1976.

6. A. J. Coleman, Induced representations with applications to $S_{n}$ and $G L(n)$, Queens Papers in Pure and Applied Math., No. 4, Queens University, Kingston, Ontario, 1966.

7. B. N. Cooperstein, Minimal degree for a permutation representation of a classical group, Israel J. Math. 30 (1978), 213-235.

8. C. W. Curtis, N. Iwahori and R. Kilmoyer, Hecke algebras and characters of parabolic type of finite groups with ( $B, N)$-pairs, Publ. Math. Debrecen 40 (1972), 81-116.

9. C. W. Curtis, W. M. Kantor and G. M. Seitz, The 2-transitive permutation representations of the finite Chevalley groups, Trans. Amer. Math. Soc. 218 (1976), 1-57.

10. P. Dembowski, Finite geometries, Springer-Verlag, Berlin and New York, 1968.

11. L. E. Dickson, Determination of all the subgroups of the known simple group of order 25920, Trans. Amer. Math. Soc. 5 (1904), 126-166.

12. __ Linear groups, Dover, New York, 1958.

13. J. Dieudonné, La géométrie des groupes classiques, Springer-Verlag, Berlin and New York, 1963.

14. L. DiMartino and A. Wagner, The irreducible subgroups of $\operatorname{PS} L\left(V_{5}, q\right)$, where $q$ is odd (to appear).

15. W. L. Edge, A permutation representation of the group of the bitangents, J. London Math. Soc. 36 (1961), 340-344.

16. L. Finkelstein, The maximal subgroups of Conway's group $C_{3}$ and McLaughlin's group, J. Algebra 25 (1973), 58-89.

17. B. Fischer, Finite groups generated by 3-transpositions. I, Invent. Math. 13 (1971), 232-246.

18. D. Flesner, Maximal subgroups of $\mathrm{PSp}_{4}\left(2^{n}\right)$ containing central elations or noncentered skew elations, Illinois J. Math. 19 (1975), 247-268.

19. J. S. Frame, The classes and representations of the groups of 27 lines and 28 bitangents, Ann. Mat. Pura Appl. (4) 32 (1951), 83-119.

20. L. Geissinger and D. Kinch, Representations of the hyper-octahedral groups, J. Algebra 53 (1978), $1-20$.

21. E. M. Hartley, Two maximal subgroups of a collineation group in five dimensions, Proc. Cambridge Philos. Soc. 46 (1950), 555-569.

22. R. W. Hartley, Determination of the ternary collineation groups whose coefficients lie in the $G F\left(2^{n}\right)$, Ann. of Math. 27 (1926), 140-158.

23. D. G. Higman, Flag-transitive collineation groups of finite projective spaces, Illinois J. Math. 6 (1962), 434-446.

24. Primitive rank 3 groups with a prime subdegree, Math. Z. 91 (1966), 70-86. 
25. D. G. Higman and J. McLaughlin, Rank 3 subgroups of finite symplectic and unitary groups, J. Reine Angew Math. 218 (1965), 174-189.

26. P. N. Hoefsmit, Representations of Hecke algebras of finite groups with BN-pairs of classical type,

$\mathrm{Ph}$.D. Thesis, Univ. of British Columbia, 1974.

27. R. B. Howlett, On the degrees of Steinberg characters of Chevalley groups, Math. Z. 135 (1974), $125-135$

28. I. M. Isaacs, Character theory of finite groups, Academic Press, New York, 1976.

29. W. Jones, Cohomology of finite groups of Lie type, Ph.D. Thesis, Univ. of Minnesota, 1975.

30. W. Jones and B. Parshall, On the 1-cohomology of finite groups of Lie type (Proc. Conf. Finite Groups, 1975), Edited by W. R. Scott and F. Gross, Academic Press, New York, 1976, pp. 313-327.

31. W. M. Kantor, Line-transitive collineation groups of finite projective spaces, Israel J. Math. 14 (1973), $229-235$.

32. On 2-transitive collineation groups of finite projective spaces, Pacific J. Math. 4 (1973), $119-131$

33. Subgroups of classical groups generated by long root elements, Trans. Amer. Math. Soc. 248 (1979), 347-379.

34. A. Kerber, Representations of permutation groups. I, II, Lecture Notes in Math., vol. 240, vol. 495, Springer-Verlag, Berlin and New York, 1970, 1975.

35. S. J. Mayer, On the characters of the Weyl groups of type C, J. Algebra 33 (1975), 59-67.

36. J. McLaughlin, Some groups generated by transvections, Arch. Math. 18 (1967), 364-368.

37. _ Some subgroups of $S L_{n}\left(F_{2}\right)$, Illinois J. Math. 13 (1969), 108-115.

38. A simple group of order 898, 128, 000, Theory of Finite Groups, edited by R. Brauer and

C.-H. Sah, Benjamin, New York, 1969, pp. 109-111.

39. unpublished.

40. H. H. Mitchell, Determination of the ordinary and modular ternary linear groups, Trans. Amer. Math. Soc. 12 (1911), 207-242.

41. . The subgroups of the quaternary abelian linear group, Trans. Amer. Math. Soc. 15 (1914), $379-396$

42. F. Murnaghan, The theory of group representations, Johns Hopkins, Baltimore, Md, 1938.

43. B. Mwene, On the subgroups of the groups $\operatorname{PSL}_{4}\left(2^{m}\right)$, J. Algebra 41 (1976), 79-107.

44. On some subgroups of $P S L(4, q), q$ odd (to appear).

45. T. Nakayama, On some modular properties of irreducible representations of a symmetric group. I, II, Japan J. Math. 17 (1941), 165-184, 411-423.

46. D. Perin, On collineation groups of finite projective spaces, Math. Z. 126 (1972), 135-142.

47. H. Pollatsek, First cohomologv groups of some linear groups over fields of characteristic two, Illinois J. Math. 15 (1971), 393-417.

48. _ . First cohomologv groups of some orthogonal groups, J. Algebra 28 (1974), 477-483.

49. unpublished.

50. G. M. Seitz, Flag-transitive subgroups of Chevalley groups, Ann. of Math. (2) 97 (1973), 27-56; Correction (to appear).

51. Small rank permutation representations of finite Chevalley groups, J. Algebra 28 (1974), $508-517$.

52. E. Snapper, Group characters and nonnegative integral matrices, J. Algebra 19 (1971), 520-535.

53. B. S. Stark, Some subgroups of $\Omega(V)$ generated by groups of root type, J. Algebra 29 (1974), 33-41.

54. , Rank 3 subgroups of orthogonal groups, Illinois J. Math. 19 (1975), 116-121.

55. R. Steinberg, A geometric approach to the representations of the full linear group over a Galois field, Trans. Amer. Math. Soc. 71 (1951), 274-282.

56. M. Suzuki, A simple group of order $448,345,497,600$, Theory of Finite Groups, edited by R. Brauer and C.-H. Sah, Benjamin, New York, 1969, pp. 113-119.

57. J. Tits, Sur la trialité et certains groupes qui s'en déduisent, Inst. Hautes Études Sci. Publ. Math. 2 (1959), 14-60.

58. Buildings of spherical type and finite BN-pairs, Lecture Notes in Math., vol. 386, Springer-Verlag, Berlin and New York, 1974.

59. A. Wagner, On collineation groups of finite projective spaces. I, Math. Z. 76 (1961), 411-426.

60. Collineation groups generated by homologies of order greater than 2, Geom. Dedicata 7 (1978), 387-398.

61. The subgroups of PSL $\left(5,2^{a}\right)$, Resultate Math. 1 (1979), 207-226. 
62. H. Wielandt, Finite permutation groups, Academic Press, New York and London, 1964.

63. A. Yanushka, Generalized hexagons of order $(t, t)$, Israel J. Math. 23 (1976), 309-324.

64. A. E. Zalesskiī, $A$ classification of the finite irreducible linear groups of degree 5 over a field of characteristic other than 0, 2, 3, 5, Dokl. Akad. Nauk BSSR 20 (1976), 773-775, 858. (Russian)

65. Classification of finite linear groups of degree 4 and 5 over fields of characteristic 2, Dokl. Akad. Nauk BSSR 21 (1977), 389-392, 475. (Russian)

66. A. E. Zalesskii and I. D. Suprenenko, Classification of finite irreducible linear groups of degree 4 over a field of characteristic $p>5$, Izv. Akad. Nauk BSSR 1978 (1978), 9-15. (Russian)

67. K. Zsigmondy, Zur Theorie der Potenzreste, Monatsh. Math. Phys. 3 (1892), 265-284.

Department of Mathematics, University of Oregon, Eugene, Oregon 97403

Department of Mathematics, Colorado State University, Fort Collins, Colorado 80523 